\title{
DYNAMICS OF CURRENT-BASED, POISSON DRIVEN, INTEGRATE-AND-FIRE NEURONAL NETWORKS*
}

\author{
KATHERINE A. NEWHALL ${ }^{\dagger}$, GREGOR KOVAČIČ $\check{L}^{\ddagger}$, PETER R. KRAMER $^{\S}$, DOUGLAS \\ ZHOU ${ }^{\llbracket}$, AADITYA V. RANGAN"I, AND DAVID CAI**
}

Dedicated to the sixtieth birthday of Professor Andrew Majda

\begin{abstract}
Synchronous and asynchronous dynamics in all-to-all coupled networks of identical, excitatory, current-based, integrate-and-fire (I\&F) neurons with delta-impulse coupling currents and Poisson spike-train external drive are studied. Repeating synchronous total firing events, during which all the neurons fire simultaneously, are observed using numerical simulations and found to be the attracting state of the network for a large range of parameters. Mechanisms leading to such events are then described in two regimes of external drive: superthreshold and subthreshold. In the former, a probabilistic argument similar to the proof of the Central Limit Theorem yields the oscillation period, while in the latter, this period is analyzed via an exit time calculation utilizing a diffusion approximation of the Kolmogorov forward equation. Asynchronous dynamics are observed computationally in networks with random transmission delays. Neuronal voltage probability density functions (PDFs) and gain curves - graphs depicting the dependence of the network firing rate on the external drive strength-are analyzed using the steady solutions of the self-consistency problem for a Kolmogorov forward equation. All the voltage PDFs are obtained analytically, and asymptotic solutions for the gain curves are obtained in several physiologically relevant limits. The absence of chaotic dynamics is proved for the type of network under investigation by demonstrating convergence in time of its trajectories.
\end{abstract}

Key words. Neuronal network, synchrony, exit-time, chaos.

AMS subject classifications. $\quad 82 \mathrm{C} 31,82 \mathrm{C} 32,92 \mathrm{~B} 20,94 \mathrm{C} 99$.

\section{Introduction}

The integrate-and-fire (I\&F) model of a neuron is one of the most basic models in biology. Ever since its inception in the early twentieth century [52], its variants have been successfully used in describing the dynamics of both single neurons and neuronal networks $[16,17,37,47,91,92]$. Its utility in neuroscience has often been contrasted with the significantly more detailed Hodgkin-Huxley model [43]. Quite frequently, especially in well-conceived, parsimonious, large-scale neuronal network models and also direct numerical simulations using such models, the simplicity of the I\&F model becomes a major advantage in effectively and efficiently uncovering robust network mechanisms governing the model dynamics $[20,71,73]$. As a consequence, the I\&F model has been the focus of many theoretical and computational studies for decades.

\footnotetext{
* Received: December 27, 2008; accepted (in revised version): May 3, 2009.

${ }^{\dagger}$ Mathematical Sciences Department, Rensselaer Polytechnic Institute, 110 8th Street, Troy, NY 12180 (newhak@rpi.edu).

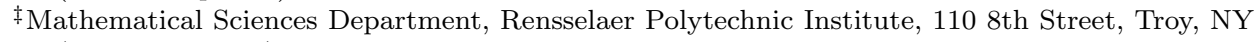
12180 (kovacg@rpi.edu).

$\S$ Mathematical Sciences Department, Rensselaer Polytechnic Institute, 110 8th Street, Troy, NY 12180 (kramep@rpi.edu).

I Courant Institute of Mathematical Sciences, New York University, 251 Mercer Street, New York, NY 10012-1185 (zdz@cims.nyu.edu).

$\|$ Courant Institute of Mathematical Sciences, New York University, 251 Mercer Street, New York, NY 10012-1185 (rangan@cims.nyu.edu).

** Courant Institute of Mathematical Sciences, New York University, 251 Mercer Street, New York, NY 10012-1185 and Department of Mathematics, Shanghai Jiao Tong University, Dong Chuan Road 800, Shanghai, China (cai@cims.nyu.edu).
} 
The simplest type of the I\&F model is the current-based model $[52,91,92]$. Like other I\&F models, it uses a forced RC circuit as a model for the dynamics of the neuron's membrane potential until this potential reaches a firing threshold. At that moment, action potentials, called spikes, are sent to all the (postsynaptic) neurons in the network connected to this original (i.e., presynaptic) neuron, whose voltage is then reset to a fixed lower value before following the $\mathrm{RC}$ circuit dynamics again. What distinguishes the current-based I\&F model is its description of how spikes are transmitted, which is as currents injected into the postsynaptic neurons, with the strength of each such current only depending on the strength of the connection between the preand postsynaptic neurons $[47,95]$. This current-based I\&F model is mathematically simpler than the conductance-based I\&F model [16]. Moreover, both the currentbased I\&F and the conductance-based I\&F model can be justified to various degrees as good and simple models for neuronal membrane potential dynamics [95].

In this paper, we consider a version of the current-based I\&F model which describes the spike-induced current injections as delta-impulses which produce an instantaneous jump in the voltages of the respective postsynaptic neurons. Despite its simplicity, this model can give a reasonably realistic description of neuron operating regimes in vivo [63] for which many spikes are needed to drive any neuron in the network to fire.

We will study network synchronization in the dynamics of all-to-all-coupled networks of excitatory, current-based, I\&F neurons and describe in detail the functioning of a synchronized network in which the neurons are driven by trains of external spikes modeled by Poisson point processes in time. A pioneering study of synchronization of current-based I\&F neurons was reported in [67], where the synchronization mechanism is described for a pair of spike-coupled neurons driven by constant superthreshold currents, i.e., currents which, without the firing and resetting mechanism, would drive the neuronal voltage to an equilibrium located above the firing threshold. For networks containing an arbitrary number of such neurons, synchronization was rigorously proven mathematically in the classic paper [55]. This result was extended to networks with constant driving force and a weak noise approximation of the discrete coupling in [50] and to parameter heterogeneity in the absence of leakage in [81]. What distinguishes the current work is the stochastic aspect brought about by the Poisson-train external drive, which is in fact the standard external drive in many neuronal network models, and carries with it a fair amount of physiological realism $[20,54,71,84,88]$. This is because the external input to each network neuron can be modeled as a train of spikes arriving from a large number of neurons outside the network under study, and these neurons fire infrequently and independently of each other [25]. Another recent paper [26] examines network synchronization in a complementary neuron model with Poissonian driving but with discrete voltage levels and no leak.

One might expect that synchrony persists even in randomly driven I\&F neuronal networks, at least as long as the average external driving currents are not too far below threshold (in the sense described in the previous paragraph) and their fluctuations are not too large. However, in this case the mathematical methods used for describing the synchrony are probabilistic in nature and thus very different from the dynamical systems method used in [55]. A large part of this work is concerned with the characterization of synchrony through total firing events, i.e., all the neurons in the network firing simultaneously, as well as the underlying mechanism of this synchrony. As will be described in detail below, this synchrony mechanism arises from the fluctuations of neuronal voltages as follows: Consider all neuronal voltages beginning 
at a reset voltage. As time progresses, the external current, a train of independent Poisson distributed spikes, causes each neuron's voltage to increase; the distribution of neuronal voltages broadens and moves towards threshold voltage. When the neuron with the largest voltage reaches threshold and fires, it increases the voltages of the remaining neurons, causing the few neurons just below threshold to fire, immediately increasing the remaining neuronal voltages and so forth until all neurons have fired. After this total firing event all neuronal voltages are at reset voltage, identical to the state in which this cycle began. The process continues to repeat itself, total firing events occurring periodically on average.

The synchrony described in the previous paragraph can occur in two different driving regimes: super- and subthreshold. The superthreshold regime is a generalization to Poisson-train driving of the deterministic or weakly-noisy situation described in $[50,55,81]$. In this case, a relatively simple probabilistic argument, related to the proof of the Central Limit Theorem [32], allows us to obtain the evolution of the probability density function (PDF) of the neuron's voltage which travels upward past the firing threshold under the influence of the Poisson-train drive. If the fluctuations in this drive are sufficiently small, one anticipates periodic total firing events. Similar to the constant current situation [55], the period with which the total firing events repeat themselves on average can be approximated as the expected time when the average of the highest neuronal voltage in the network crosses the threshold. In addition, we develop a cascade-susceptibility criterion for a total firing event to occur, and use this to compute the probability of total firing events to occur in succession.

Analysis of the subthreshold regime is considerably more involved since the mean of the external drive no longer brings a neuronal voltage past the firing threshold on average. Instead, it is pushed over the threshold by fluctuations in the external drive current, and a delicate balance between the sizes of these fluctuations and the mean external current must be struck in order to ensure the existence of the total firing events on the one hand and their approximate periodicity on the other. We obtain the description of the dynamics in this regime, in particular, the average period between two consecutive total firing events, via the solution of a first-passage-time problem using a diffusion approximation of the Kolmogorov forward equation (KFE) [35]. This solution is given by a series involving the eigenfunctions of a non-self-adjoint operator. These eigenfunctions are expressed in terms of the confluent hypergeometric functions [83], and the coefficients in the series are obtained by integrating the infinitely narrow initial PDF against the bi-orthogonal eigenfunction sequence of the adjoint operator. We also present an alternative derivation of the KFE, to provide a different perspective from [21].

We contrast the oscillatory regime of the current-based I\&F network with its asynchronous regime $[1,14,50]$. Our numerical simulations indicate that this asynchronous regime is difficult to reach when the drive is superthreshold as it appears to have a very small basin of attraction. We obtain a broader asynchronous regime by including in the model some physiological effects that break the conditions for synchrony. This can be achieved in several ways, including sparsity of neuron-to-neuron connections $[5,13,93,94]$, random synaptic failure $[3,41,62,69,74,96]$, or random synaptic transmission delays $[12,29,56]$, which we use in the present work. We describe the steady-state of this regime using again the diffusion approximation of the KFE, for which the voltage PDFs as well as gain curves (i.e., firing rate versus average external drive strength) are obtained exactly by refining the analysis of [14]. In the regime in which the external drive has small fluctuations, the gain curves exhibit bistability and 
hysteresis, the shape of which can be characterized very precisely in the limit when the fluctuations vanish. In this limit, the stationary KFE becomes an algebraic equation, similar to those studied in [50], which can easily be integrated to yield the gain curve. We find an explicit solution describing the gain curves and all their relevant characteristics analytically. In the case when the external drive fluctuations are small but non-vanishing, two asymptotic regimes are characterized and exact solutions for both the voltage PDF and the gain curves are obtained.

Finally, we address the question of chaotic dynamics in current-based I\&F neuronal networks with delta-impulse current interactions driven by spike trains. It is well known that individual Hodkin-Huxley neurons can exhibit chaotic dynamics, for example under periodic drive $[39,99]$. It was recently shown that networks of such neurons can collectively exhibit chaotic dynamics (by possessing positive Lyapunov exponents) even when each individual neuron exhibits no chaos in the absence of network coupling. This demonstrated that network coupling can induce chaos even when each individual component of the network possesses only regular dynamics [86, 87]. Clearly, a single current-based I\&F neuron cannot become chaotic under any drive, and it was recently shown that a single conductance-based I\&F neuron cannot either, provided the firing threshold is kept constant [10]. On the other hand, for excitatory, conductance-based, I\&F neuronal networks with a finite conductance time course, a chaotic regime was found [101]. Here, we show that spike train driven current-based I\&F neuronal networks with delta-impulse interactions cannot exhibit chaotic dynamics, and that, in fact, two nearby trajectories of such a network generically should coalesce after a finite time. This implies the possible existence of a strongly attracting non-chaotic random attractor (under stochastic Poisson driving), which we conjecture to be synchronized or near-synchronized in some probabilistic sense. Investigating the precise nature of this attractor will be relegated to future work.

The remainder of this paper is organized as follows. In section 2 , we describe the I\&F model under investigation and define a number of its operating regimes and limits. In section 3, we discuss synchronous dynamics of the model and define the notion of total firing events. We begin with the superthreshold regime, and develop a probabilistic description of the neuronal voltages between two sequential total firing events which is then used to obtain the average frequency of such events. For this regime, we also probabilistically determine the parameter ranges in which these events are likely to happen. We then proceed to the subthreshold regime, where we present the dependence of the expected time between total firing events and the voltage PDF of a single neuron. We continue by deriving the appropriate Kolmogorov forward and backward equations (KFE and KBE) and their diffusion approximations, the solutions of which we construct with a series of confluent hypergeometric functions. For both the superand subthreshold regimes, the predicted firing rates are compared to measurements from numerical simulations. In section 4 , we describe the asynchronous regime of network operation, beginning with a description of what physiological effects may break the tendency of the network to synchronize. We proceed by deriving the steady KFE for the voltage PDF in the asynchronous regime and describing the solutions of this steady KFE and the corresponding gain curves in the zero-fluctuation limit, as well as the exact solutions, gain curves, and asymptotic formulas in two complementary regimes for the case of small fluctuations. In section 5, we prove that there are no chaotic dynamics in the current-based I\&F network with delta-pulse couplings driven by spike trains. We present a discussion and the conclusions in section 6 . In the appendix, we describe the details of several calculations used in section 3.2: in ap- 
pendix A, we derive a stationary PDF used in the calculation of the eigenfunctions for the KFE and $\mathrm{KBE}$, in appendix $\mathrm{B}$, we show how to solve both the KFE and KBE simultaneously, and in appendix $\mathrm{C}$, we present the computational details needed to evaluate the solution for the expected time between total firing events.

\section{The model}

We consider a model neuronal network of $N$ all-to-all coupled, current-based, excitatory, integrate-and-fire (I\&F) point neurons. This network is governed by the system of differential equations

$$
\frac{d v_{j}}{d t}=-g_{L}\left(v_{j}-V_{R}\right)+I_{j}(t), \quad j=1, \ldots, N,
$$

where $v_{j}$ is the membrane potential of the $j^{\text {th }}$ neuron, $g_{L}$ is the leakage conductance, $V_{R}$ is the resting voltage, and $I_{j}(t)$ is the injected current. The voltage, $v_{j}$, evolves according to equation (2.1) while it remains below the firing threshold, $V_{T}$. When $v_{j}$ reaches $V_{T}$, the $j^{\text {th }}$ neuron is said to fire a spike, and $v_{j}$ is set to the value of the reset voltage, which is $V_{R}$ in this work. Upon resetting, $v_{j}$ is immediately governed by equation (2.1) again. At the same time, appropriate currents are injected into all other neurons as described below. For the purpose of comparison to numerical simulation, the non-dimensional values

$$
V_{R}=0, \quad V_{T}=1, \quad \text { and } \quad g_{L}=1
$$

are used. Note that the leakage conductance, $g_{L}$, sets the time scale.

The instantaneous currents that drive the $j^{\text {th }}$ neuron in the network (2.1) are modeled by the expression

$$
I_{j}(t)=f \sum_{l} \delta\left(t-s_{j l}\right)+\frac{S}{N} \sum_{i \neq j} \sum_{k} \delta\left(t-\tau_{i k}\right),
$$

where $\delta(\cdot)$ is the Dirac delta function. The first term in equation (2.3) corresponds to the currents arriving from the external input. Each neuron's external input is modeled by a Poisson train of Dirac delta function spikes with rate $\nu$. At each spike time, $t=s_{j l}$, the neuron's voltage jumps by an amount $f$. The sum for the $j^{\text {th }}$ neuron is over all spike times, $s_{j l}$, which are statistically independent from the times $s_{i l}$ for any other neuron $i \neq j$. The second term in equation (2.3) corresponds to the coupling between pairs of neurons in the network. For this all-to-all coupled network, a spike generated by a neuron is modeled by a Dirac delta function in time which causes the voltages of all other neurons in the network to jump by the amount $S / N$. The scaling by $N$, the number of neurons in the network, ensures the average firing rate per neuron remains bounded as $N \rightarrow \infty$. The time $\tau_{i k}$ corresponds to the $k^{\text {th }}$ time the $i^{\text {th }}$ neuron's voltage reaches firing threshold, $V_{T}$. The sum is over all other spiking neurons except the $j^{\text {th }}$ neuron itself.

Each Poisson point process that generates the set of spike times $\left\{s_{j l}\right\}_{l=1}^{\infty}$ for the external input to the $j^{\text {th }}$ neuron is independent from the Poisson point processes for the other $N-1$ neurons. The Poisson point processes are all statistically identical, each defined on the non-negative real axis, $[0, \infty)$, with rate $\nu$. Corresponding to each of these Poisson point processes, we also define a Poisson counting process, $M_{j}(t)$, to describe the number of spikes from the external input appearing in the $j^{\text {th }}$ neuronal current during the time interval $[0, t]$. These $M_{j}(t)$ are independent 
stochastic processes with Poisson distributions at each moment of $t$ :

$$
P\left(M_{j}(t)=m\right)=\frac{(\nu t)^{m}}{m !} e^{-\nu t}
$$

with mean $\nu t$. These external spike times generated for a single neuron in one time interval are independent from those times in any other non-overlapping time interval $[44$, Sec. $1.3 f]$.

Simulations of the above neuronal network model are carried out by solving equations (2.1) and (2.3) exactly. Time stepping is controlled by the next external spike time across the network. If this spike is capable of increasing the neuron's voltage above threshold, this neuron fires, and the other neuronal voltages are all increased instantaneously. Then, the remaining neuronal voltages are examined to see if any are above threshold. If other neuronal voltages are above threshold, these neurons fire, and then the remaining voltages (of neurons which have not fired) are increased appropriately. This process repeats until no new neurons spike. Then, we return to the time stepping procedure and repeat. Under the dynamics of equation (2.3), a neuron can only fire when an incoming spike arrives. This simulation procedure is similar to the one discussed with more detail in [11]. Synchrony appears in the simulations for sufficiently large network coupling strength, $S$, and large Poisson rate, $\nu$. Examples of synchronous dynamics of the network (2.1) are shown in figure 2.1.

We should note that the driving effect on the network (2.1) of an external Poisson spike train with rate $\nu$ and spike strength $f$ in the limit as $f \rightarrow 0$ and $\nu \rightarrow \infty$, by the law of large numbers, is the same as that of the constant current of strength $f \nu$. We will refer to the limit as $f \rightarrow 0$ and $\nu \rightarrow \infty$ while $f \nu=O(1)$ as the zero-fluctuation limit. In section 3.1.1 we show the fluctuations are $O\left(f^{2} \nu\right)$ which are $O(f)$ when $f \nu=O(1)$. Most results presented below are for the situation when $f$ is small and $\nu$ large, with $f \nu=O(1)$, which we will refer to as the small-fluctation regime.

When the network coupling is large in our simulations, not only does it sustain the synchrony in the network dynamics, but it drives an unsynchronized network into the synchronous state, as will be discussed in section 3.1.3. This is illustrated in figure 2.1 for networks with voltages initially uniformly randomly distributed between $V_{R}$ and $V_{T}$. When the network coupling is sufficiently large that the network is completely synchronized, the network with randomly distributed voltages does not exhibit any transition period; it begins firing immediately with total firing events. We note that the network coupling strength, regarded as being sufficiently large for the purpose of synchrony, is still weak in the sense that it requires many incoming spikes from other neurons to cause a neuron to fire, that is, $S / N \ll V_{T}-V_{R}$.

In what is to follow, we will address two distinct regimes of network operation: subthreshold, and superthreshold. These regimes are characterized by considering the network (2.1) with fluctuating external current replaced by its average, $f \nu$, and the network coupling turned off, $S=0$. This is equivalent to a single neuron driven with constant external current of strength $f \nu$. The full network is in the subthreshold regime whenever $f \nu<g_{L}\left(V_{T}-V_{R}\right)$, under which condition the voltage of the single neuron with constant current and zero network coupling will not cross threshold, and thus never fire. On the other hand, the superthreshold regime is used to describe the network dynamics whenever $f \nu>g_{L}\left(V_{T}-V_{R}\right)$. In this case, the voltage of the single neuron with constant current and zero network coupling is driven above threshold, and will fire repeatedly at regular intervals of time.

We also discuss two regimes related to the effects of fluctuation in the input current: fluctuation-driven regime and mean-driven regime. In the fluctuation-driven 

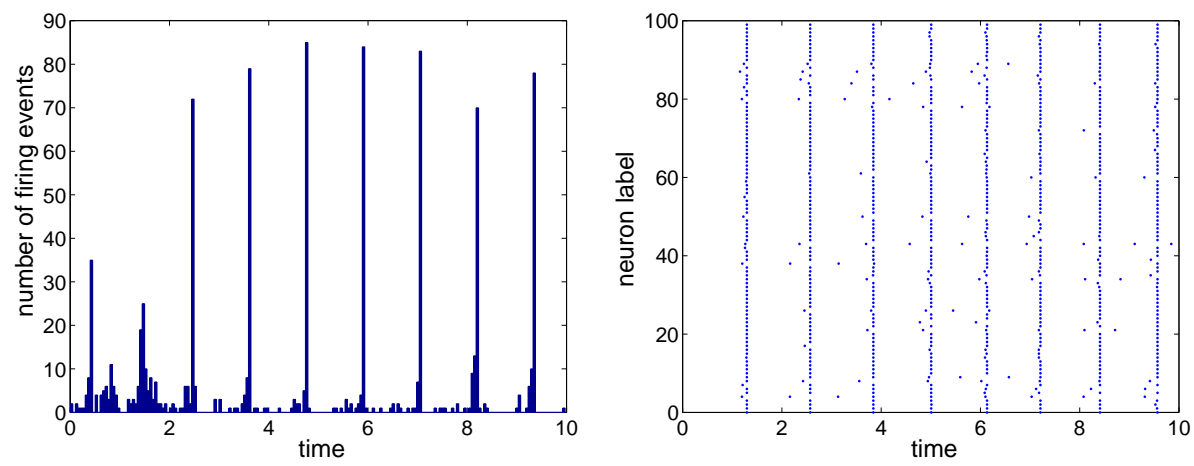

(a) $f=0.01, f \nu=1.2, S=0.4$ and $P(C)=0.00027$
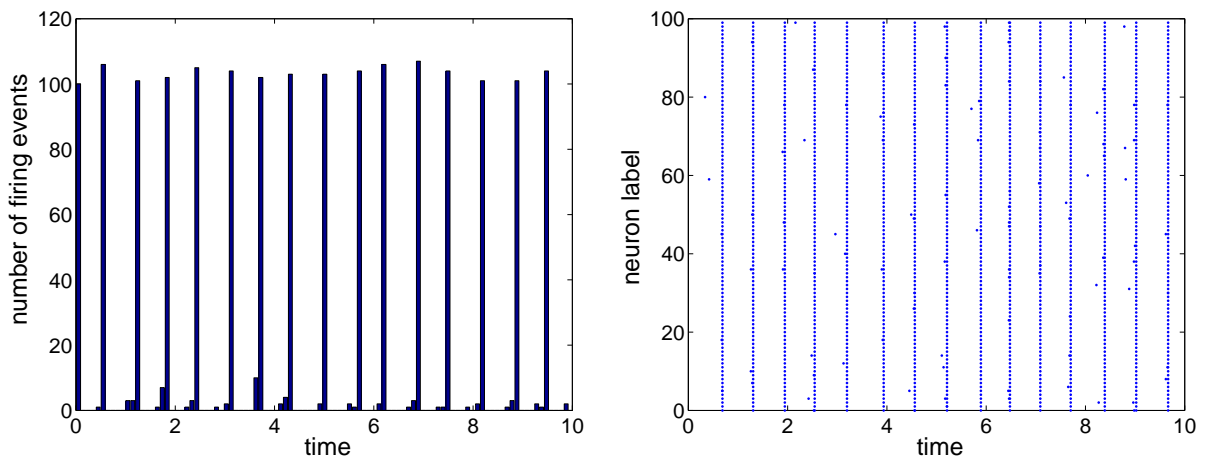

(b) $f=0.1, f \nu=1.2, S=2.0$ and $P(C)=0.0034$
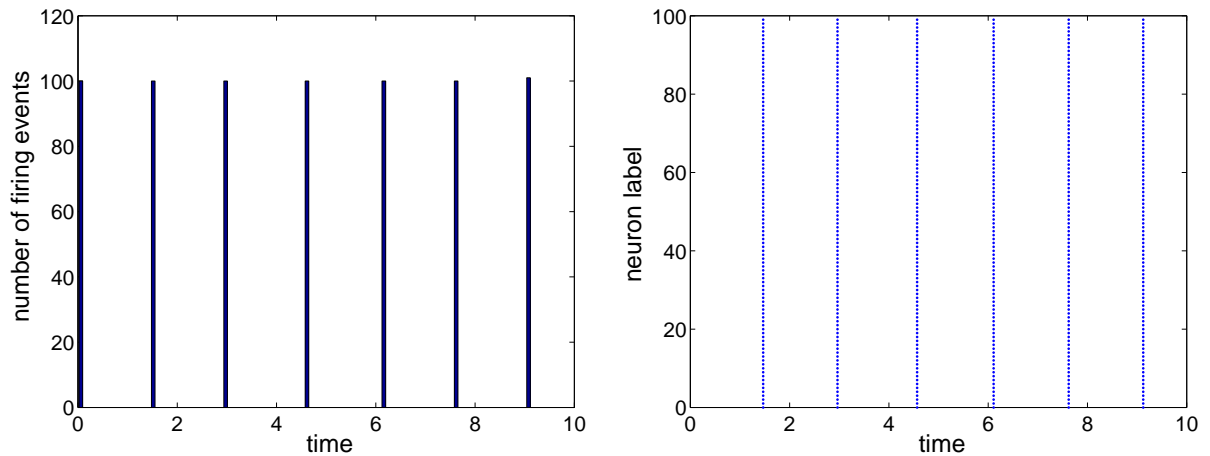

(c) $f=0.001, f \nu=1.2, S=2.0$ and $P(C)=0.99$

FIG. 2.1. (Left) Histograms of firing times for the system with uniformly randomly distributed initial voltages illustrating the trend toward synchronous firing. (Right) Raster plots of firing times for the system with initial voltages set at $V_{R}$. For the synchronizable network (c), one total firing event is followed by another total firing event with high probability. The other two systems, (a) and (b), do not satisfy our stringent definition of synchronizability. 
regime, the activities of the full network are driven by fluctuations about the mean voltage. In the mean-driven regime, the entire network is driven by the mean of the input current.

\section{Synchrony}

The network synchronizes exactly through total firing events. These synchronized events are manifested as vertical lines in the raster plots in figure 2.1, where dots indicate which neuron fired at what time. Total firing events are cascading events during which all neurons in the network fire simultaneously, and all neuronal voltages, $v_{j}$, are reset to $V_{R}$ at the same time. The voltages then rise probabilistically due to the external driving, until the first neuron fires, and pushes all other neurons to fire with it. The distribution of neuronal voltages is classified as cascade-susceptible, if the firing of one neuron activates a total firing event. The system is then classified as $p$-synchronizable if the probability of neuronal voltages to be cascade-susceptible is greater than $p$ when the first neuron fires. In the discussion below, we use $p=$ 0.85 throughout and call $p$-synchronizable simply synchronizable. By this definition the networks (a) and (b) in figure 2.1 are not synchronizable, as other firing events appear which are not part of the total firing events. We remark that this definition of synchronizability is rather stringent. The networks in figure 2.1 (a) and (b) might be considered synchronous by some other broader definition.

From equations (2.1) and (2.3), it follows that the $j^{\text {th }}$ neuronal voltage, $v_{j}$, can only increase when that neuron receives a spike. At that time, the voltage $v_{j}$ jumps up by $f$ or $S / N$, respectively, depending on whether the arriving spike was generated by the external input or another neuron in the network. If $v_{j}$ exceeds the firing threshold, $V_{T}$, during such a jump, the $j^{\text {th }}$ neuron will fire, and an amount $S / N$ is added to the voltage of all the other neurons. At this same instant, the increment of $S / N$ may increase the $k^{\text {th }}$ neuronal voltage above threshold, $V_{T}$, and it will fire, and so on, and a cascading firing event may thus take place. This cascading effect maintains the synchrony of the network. In particular, it keeps the network exactly synchronized if the neuronal voltages are all sufficiently close to one another such that the cascading event includes all neurons in the network, giving rise to a total firing event. The probability of a total firing event occurring depends on the network coupling constant, $S$, and is computed in section 3.1.3.

After a total firing event, all the neuronal voltages are reset to $V_{R}$, and then rise independently due to their independent external Poisson spike trains until the first spiking of a network neuron. The voltages of all the neurons in the network are independent since between total firing events no neurons are firing, thus the network coupling is effectively zero. The expected time between total firing events, and thus the spike rate of the network, is the expected time, starting from the reset moment, for the first neuron to fire. In section 3.1 and 3.2, the expected first exit time will be calculated with different techniques for the subthreshold and superthreshold regimes.

3.1. Superthreshold regime. The time between total firing events in the synchronous network is determined by the time for the first neuron to reach threshold voltage and begin the cascade firing event. In contrast to the zero-fluctuation limit method for the asynchronous model [21], we consider the small fluctuations and compute the distribution of voltage for the neuron with the maximum voltage among all neuron voltages in the network, then we approximate the mean time this neuron with maximal voltage exits by determining when the mean of the maximal voltage crosses the threshold. In this approach we extract the properties of the dynamics from the mean of the maximum neuronal voltage. 
The average firing rate of the synchronous network will be approximated in terms of the average time the maximal voltage of all $N$ neurons crosses threshold. In order to determine this time, we will derive the PDF for the voltage of the neurons in the network between total firing events, and show that this PDF can be approximated by the Gaussian distribution. Then in section 3.1.2 we obtain the PDF for the maximum neuronal voltage in the network from the PDF for a single neuronal voltage. From this PDF of the maximal voltage, the expectation of the maximum voltage is computed numerically and used to approximate the average time this neuron crosses threshold voltage.

3.1.1. Voltage distribution for uncoupled neurons. The mechanism for synchrony in the superthreshold driving regime is related to the distribution of the neuronal voltages, resulting from their random inputs, described by equation (2.3). As mentioned before, these inputs are generated by two sources: the external input modeled by a Poisson point process and the internal input from other neurons in the network firing. In the synchronous state, no neurons fire between total firing events, thus all the input to a given neuron is generated by the external input. The differential equation in (2.1) which describes the membrane potential, $v_{j}$, of any neuron in the network at time $t$, now takes the form

$$
\frac{d v_{j}}{d t}=-g_{L}\left(v_{j}-V_{R}\right)+\sum_{l} f \delta\left(t-s_{j l}\right)
$$

between any two consecutive total firing events when the network is in the synchronous state.

The Poisson point process defining the incoming spike times $\left\{s_{j l}\right\}$ has constant rate, therefore the system (3.1) is homogeneous in time, and the initial conditions $v_{j}(0)=V_{R}, j=1, \ldots, N$, can be assigned after any total firing event. The solution to the linear inhomogeneous differential equation (3.1) is

$$
v_{j}(t)=V_{R}+f \sum_{l=1}^{M(t)} e^{-g_{L}\left(t-s_{j l}\right)},
$$

provided that $v_{j}$ has remained below $V_{T}$ in the interval $[0, t]$. As the rate of Poisson points is the same for all neurons, equation (3.2) is simply a different realization of the Poisson point process for each neuron, and is thus statistically identical for all neurons. The subscript $j$ will be dropped for the remainder of this discussion.

In order to compute the period of the total firing events taking place in our model, we first need to compute the PDF of the neuronal voltage (3.2). We cannot find it in closed form, and so we will only seek it in the small-fluctuation regime, which, we recall from section 2 , is characterized by high incoming-spike Poission rate, $\nu$, and small spike strength, $f$, such that the overall driving strength, $f \nu$, remains constant.

We proceed in several steps. First, we note that for the purposes of computing the statistics of the neuronal voltage at any given single time $t$, we can rewrite equation (3.2) in terms of a sum involving independent and unordered spike times $U_{l}(t)$ as [44, Sec. 4.2B]

$$
v(t)=V_{R}+\sum_{l=1}^{M(t)} R_{l}(t)=V_{R}+\sum_{l=1}^{M(t)} f e^{-g_{L}\left(t-U_{l}(t)\right)} .
$$


Each $U_{l}(t)$ is uniformly distributed on the interval $[0, t]$ with $\operatorname{PDF} p_{U(t)}(u)=1 / t$ for $u \in[0, t]$ and zero elsewhere. Next, consider the random variable $R_{l}(t)$, which takes values in the interval $\left[f e^{-g_{L} t}, f\right]$. The $R_{l}(t)$ 's are identically distributed, and the PDF for each $R_{l}(t)$ can be derived from the PDF of $U_{l}(t)$ by considering $R_{l}(t)$ as a function of $U, R(t)=g(U(t))=f e^{-g_{L}(t-U(t))}$, through the transformation rule [7]

$$
p_{R(t)}(r)=\frac{p_{U(t)}\left(g^{-1}(r)\right)}{\left|g^{\prime}\left(g^{-1}(r)\right)\right|},
$$

where the prime denotes differentiation with respect to the argument of the function $g(\cdot)$. This mapping is one to one. This yields the PDF for $R_{l}(t)$ to be

$$
p_{R(t)}(r)=\frac{1}{g_{L} t} \frac{1}{r} \quad \text { for } \quad r \in\left[f e^{-g_{L} t}, f\right]
$$

and zero elsewhere.

According to equation (3.3), each neuron's voltage is given by the equation $v(t)-V_{R}=\sum_{l=1}^{M(t)} R_{l}(t)$, which is a sum of a random number of independent identically distributed random variables. From equations (2.4) and (3.5), using the formula for the mean of random sums [44, Sec. 1.1e], we thus compute the average neuronal voltage at the time $t$ to be

$$
\mu(t)=V_{R}+\langle M(t)\rangle\left\langle R_{l}(t)\right\rangle=V_{R}+\frac{f \nu}{g_{L}}\left(1-e^{-g_{L} t}\right),
$$

where we have taken into account that

$$
\langle M(t)\rangle=\nu t \quad \text { and } \quad\left\langle R_{l}(t)\right\rangle=\frac{f}{g_{L} t}\left(1-e^{-g_{L} t}\right) .
$$

The PDF of the voltage, $v(t)$, would look like a delta function in the zerofluctuation limit because its variance would vanish. To describe the mean as well as the fluctuations of the voltage in this limit, we center the voltage about its mean, rescale by the amplitude of the fluctuations, and define

$$
\begin{aligned}
w(t)=\frac{v(t)-\mu(t)}{f \sqrt{\nu}} & =\frac{1}{f \sqrt{\nu}} \sum_{l=1}^{M(t)} R_{l}(t)-\frac{\langle M(t)\rangle\left\langle R_{l}(t)\right\rangle}{f \sqrt{\nu}} \\
& =\sum_{l=1}^{M(t)} H_{l}(t)-m_{r}(t),
\end{aligned}
$$

where

$$
m_{r}(t)=\frac{\sqrt{\nu}}{g_{L}}\left(1-e^{-g_{L} t}\right) .
$$

Since $w(t)$ is a random sum of independent, identically distributed random variables, $H_{l}(t)=(f \sqrt{\nu})^{-1} R_{l}(t)$, the methods of characteristic functions and generating functions are invoked for the computation of the PDF for $w(t)$.

The characteristic function of a random variable $X$ is defined as the Fourier transform of its probability distribution [44, Sec. 1.1d],

$$
\phi_{X}(\kappa)=\left\langle e^{i \kappa X}\right\rangle
$$


For a random sum of $M(t)$ independent, identically distributed random variables $H_{l}(t)$, the characteristic function equals the composite function [44, Sec. 1.1e] (shifted by the additive deterministic term)

$$
\phi_{w(t)}(\kappa)=e^{-i \kappa m_{r}(t)} G_{M(t)}\left(\phi_{H(t)}(\kappa)\right),
$$

where

$$
\begin{aligned}
\phi_{H(t)}(\kappa) & =\left\langle e^{i \kappa H(t)}\right\rangle=\int_{-\infty}^{\infty} e^{i \kappa r / f \sqrt{\nu}} p_{R(t)}(r) d r \\
& =\frac{1}{g_{L} t} \int_{f \exp \left(-g_{L} t\right)}^{f} \frac{e^{i \kappa r / f \sqrt{\nu}}}{r} d r
\end{aligned}
$$

is the characteristic function of the continuous random variable $H_{l}(t)$ and

$$
\begin{aligned}
G_{M(t)}(s) & =\left\langle s^{M(t)}\right\rangle=\sum_{m=0}^{\infty} P(M(t)=m) s^{m} \\
& =e^{-\nu t} \sum_{m=0}^{\infty} \frac{(\nu t)^{m}}{m !} s^{m}=e^{\nu t(s-1)}
\end{aligned}
$$

is the generating function of the discrete random variable $M(t)$, computed from equation (2.4). From equations (3.11), (3.12), and (3.13), we obtain the characteristic function

$$
\phi_{w(t)}(\kappa)=e^{-i \kappa m_{r}(t)} \exp \left[\nu t\left(\frac{1}{g_{L} t} \int_{f \exp \left(-g_{L} t\right)}^{f} \frac{e^{i \kappa r / f \sqrt{\nu}}}{r} d r-1\right)\right],
$$

with $m_{r}(t)$ defined in equation (3.9).

From formula (3.14), we can immediately calculate the cumulant generating function $G_{w(t)}$ of the rescaled voltage, $w(t)$, in equation (3.8) as

$$
G_{w(t)}(\kappa)=\ln \phi_{w(t)}(\kappa)=\nu t\left(\frac{1}{g_{L} t} \int_{f \exp \left(-g_{L} t\right)}^{f} \frac{e^{i \kappa r / f \sqrt{\nu}}}{r} d r-1\right)-i \kappa m_{r}(t) .
$$

From this function, the $n^{\text {th }}$ cumulant of $w(t)$ can be computed via the formula

$$
c_{n}[w(t)]=\left.(-i)^{n} \frac{d^{n} G_{w(t)}(\kappa)}{d \kappa^{n}}\right|_{\kappa=0}=\frac{1}{g_{L}} \frac{\nu}{(f \sqrt{\nu})^{n}} \int_{f \exp \left(-g_{L} t\right)}^{f} \frac{r^{n}}{r} d r \quad \text { for } n \geq 2 .
$$

These cumulants are $O\left(\nu^{1-n / 2}\right)$ since the integral in equation (3.16) is of $O\left(f^{n}\right)$. Considering $f \nu=O(1)$, this is equivalent to

$$
c_{n}[w(t)]=O\left(f^{n / 2-1}\right) .
$$

Since the first cumulant vanishes by the choice of the variable $w(t)$, the only nonvanishing cumulant in the zero-fluctuation limit is the second, which is $O(1)$. Consequently, the probability distribution for $w(t)$ in the small-fluctuation regime is exactly that for a Gaussian distribution with mean zero and variance $c_{2}[w(t)]$. A more precise examination shows that the cumulants in (3.15) scale as $\left(\nu / g_{L}\right) \nu^{-n / 2}$ and are therefore negligible for $n \geq 3$ in an appropriately nondimensionalized sense when $\nu g_{L} \gg 1$. 


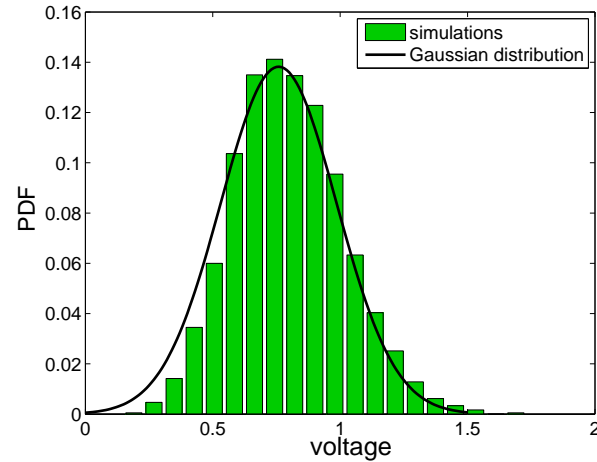

(a) $f=0.1, f \nu=1.2$ and $t=1$

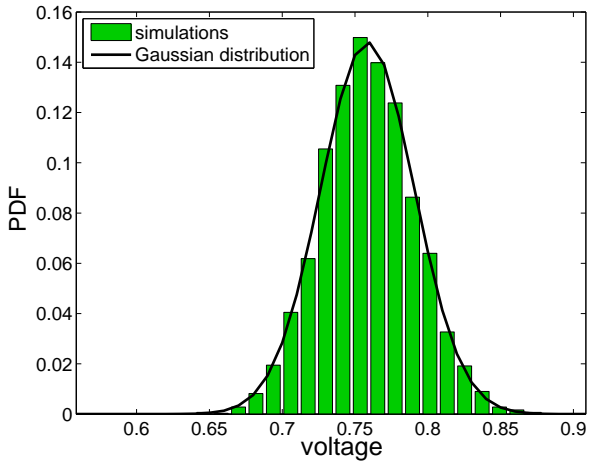

(b) $f=0.002, f \nu=1.2$ and $t=1$

FIG. 3.1. The Gaussian distribution for the neuronal voltage (equation (3.18)) is compared to the actual distribution of neuronal voltages in the network where voltages are not reset to $V_{R}$ when they cross threshold. The approximation improves for smaller $f$. (a) Large fluctuations: the Gaussian distribution remains a reasonable approximation. (b) Small fluctuations: the Gaussian distribution provides an excellent approximation over several standard deviations about the mean.

The Gaussian approximation for small fluctuations is therefore formally valid when $f \ll(f \nu) g_{L}$, where $f \nu$ is the $O(1)$ mean external current strength.

The Gaussian distribution approximation for the voltage, $v(t)$, is

$$
p_{v}(x, t) \sim \frac{1}{\sqrt{2 \pi} \sigma(t)} \exp \left(-\frac{(x-\mu(t))^{2}}{2 \sigma^{2}(t)}\right),
$$

where the average voltage, $\mu(t)$, is given in equation (3.6) and its variance is given by

$$
\sigma^{2}(t)=\frac{f^{2} \nu}{2 g_{L}}\left(1-e^{-2 g_{L} t}\right) .
$$

Later, the cumulative distribution function (CDF) of the voltage will also be needed, which for the Gaussian approximation of the PDF is given by

$$
F_{v}(x, t) \sim \frac{1}{2}\left(1+\operatorname{erf}\left(\frac{x-\mu(t)}{\sqrt{2} \sigma(t)}\right)\right),
$$

where $\operatorname{erf}(\cdot)$ is the error function,

$$
\operatorname{erf}(z)=\frac{2}{\sqrt{\pi}} \int_{0}^{z} e^{-t^{2}} d t
$$

We point out that this approximate PDF (3.18) is in good agreement with numerical simulations, as depicted in figure 3.1 (b) for uncoupled neurons in the small-fluctuation regime, the voltages of which are not reset to $V_{R}$ when they cross threshold.

3.1.2. Firing rate. The time between total firing events in the synchronized network is the time it takes for the first neuron to reach threshold. In order to compute the mean of this time now that we have a good approximation for the PDF of the voltage for a single neuron, we first derive the PDF for the maximum voltage 
of $N$ uncoupled neurons. Then we approximate the average time as the time it takes the average maximum voltage to reach threshold. The error of this approximation is negligible in the small-fluctuation regime. We remark that for networks of the size we are considering, $N \sim 100$, the largest voltage at any given time is still typically only 2 or 3 standard deviations from the mean and, as will be shown below, our procedure yields a reasonably good description of the firing rate. Therefore, we neither use a careful approximation of the tails of the probability distribution nor pursue a large deviation analysis.

Since the voltages of all the $N$ neurons in the network are independent and identically distributed random variables, the probability that all of them will be below a given value, $x$, at a given time $t$, is given by the product of the respective single-neuron voltage probabilities. In other words, the CDF of such an event is given by

$$
F_{v}^{(N)}(x, t)=F_{v}(x, t)^{N},
$$

where $F_{v}(x, t)$ is the voltage CDF for a single neuron, given by equation (3.20). From equation (3.22), we find that the PDF for the maximum voltage over the network is thus given by the expression

$$
p_{v}^{(N)}(x, t)=N p_{v}(x, t) F_{v}(x, t)^{N-1},
$$

where $p_{v}(x, t)$ is the single-neuron voltage PDF given by equation (3.18).

We approximate the expected time the first neuron crosses threshold by computing the time the expected maximum voltage equals threshold by solving the equation

$$
V_{T}=\int_{-\infty}^{\infty} x N p_{v}(x, t) F_{v}(x, t)^{N-1} d x
$$

for $t$, which appears in the $\operatorname{PDF} p_{v}(x, t)$ and the $\operatorname{CDF} F_{v}(x, t)$. The average first exit time, $t$, is computed by numerically solving equation (3.24). This approximation is valid when the standard deviation of the maximal neuronal voltage,

$$
\sigma_{(N)}=\sqrt{\int_{0}^{\infty}\left(x-\mu_{(N)}\right)^{2} N p_{v}(x, t) F_{v}(x, t)^{N-1} d x}
$$

is small in comparison to the mean, $\mu_{(N)}$, of the distribution. We consider the standard deviation of the distribution at the time its mean is $V_{T}$, with non-dimensional value unity. Over the range of values of $f$ and $\nu$ for which the Gaussian approximation is valid and the network is synchronous, the standard deviation, $\sigma_{(N)}$, is never more than $3.5 \%$ of the mean, $\mu_{(N)}$. To further validate this approximation, in figure 3.2 we compare numerical measurements of the first exit time in a simulation of the full network dynamics (2.1), to that of the mean predicted by equation (3.24). We observe good agreement between the measurement and theoretical mean for small $f$.

The gain curves depicting the frequency of the synchronized oscillations (i.e., the average network firing rate) versus the average external current, $f \nu$, obtained from this theoretical solution are shown in figure 3.3 along with the corresponding gain curves computed via numerical simulation of the network. The predicted gain curves are clearly in good agreement with the numerical measurements.

We see that, holding $f \nu$ fixed, the firing rates depend strongly on the fluctuation strength $f$. To understand the qualitative dependence, we first note that the strength of the variance (3.19) of the voltage PDFs, $p_{v}(x, t)$, scales linearly with $f$ (for fixed 

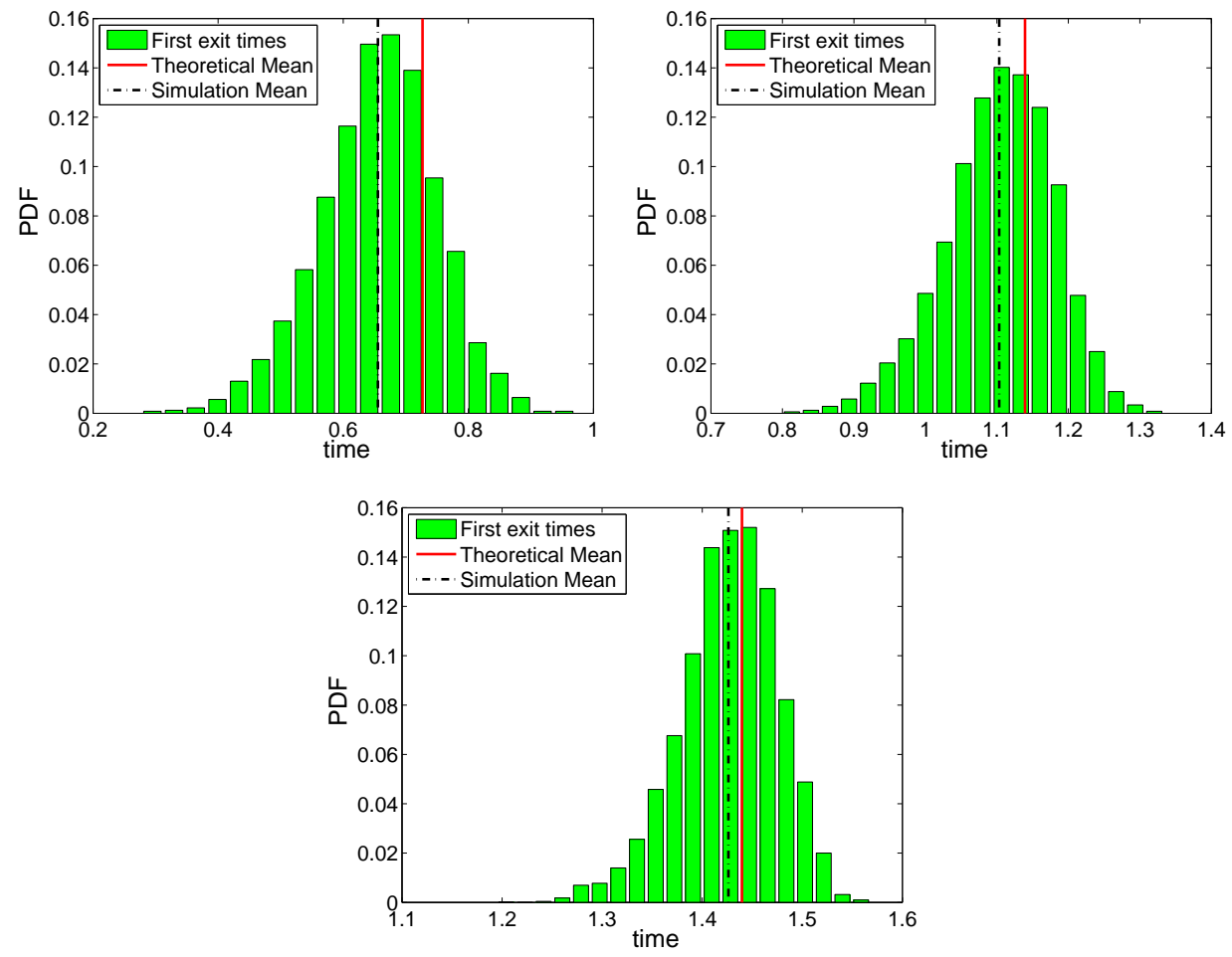

FIG. 3.2. Comparison of first exit times measured in simulations to the time predicted via equation (3.24) for $N=100$ neurons with $f \nu=1.2$. (Top left) $f=0.05$, (top right) $f=0.01$, and (bottom) $f=0.002$. The case of $f=0.05$ is just outside the small-fluctuation regime; for the other two values of $f$, the difference between the measured and theoretical means are small. Note the $x$-axis scale changes for these three cases.

$f \nu)$. Decreasing $f$ within the small-fluctuation regime tightens the $\mathrm{PDF}, p_{v}(x, t)$, of the neuronal voltages, thereby extending the amount of time needed for some neuron to reach threshold, and slowing the firing rate. This clearly indicates that the fluctuations of the voltages, not just their means, control the firing rate. In contrast, the firing rates of both a single uncoupled neuron and coupled neurons in an asynchronous dynamic regime can be obtained by an approximation that replaces the fluctuating driving force with the equivalent mean constant driving current (cf. section 4.4, especially figure 4.3). For sufficiently large $f$ for which the network dynamics are no longer in the small-fluctuation regime, as expected, the gain curves measured from the simulations begin to deviate from our theory.

The theory we have just developed contains no dependence on the network coupling strength, S, apart from the assumption that the connection is sufficiently strong so that the network dynamics are synchronous. This assumption is verified by comparing numerical simulations of networks with two different coupling strengths, both in the regime when the network displays synchronous behavior. The results are presented in figure 3.4. In the superthreshold regime, no difference is seen in the firing rate as a function of the average external current, $f \nu$. Well into the subthreshold regime, our simulations show that the networks do not synchronize as effectively, 
which explains why the numerical results deviate from the theory premised on at least approximate synchrony.

The size of the network, $N$, has a weak effect on the firing rate of the synchronous network, evident in the gain curves in figure 3.3. As the size, $N$, of the network is increased, it becomes more likely for the voltage of one neuron to be further from the mean. In turn, it becomes more likely for a neuron to reach threshold sooner, thus increasing the firing rate of the network. The difference in firing rates is small, since the typical deviation from the mean of the maximal neuron in a population of $N$ neurons grows approximately logarithmically with $N$.

The computation of the time between synchronous firing events through consideration of the expected value of the maximum neuronal voltage illuminates the fact that the synchronous events are controlled by fluctuations in the voltage of a single neuron. The more likely a single neuronal voltage is to be found further from the mean voltage of all $N$ neurons, the faster the network fires, given that the network remains synchronizable. This analytical result does not capture the dynamics of the system driven by inputs below threshold. We will consider this network regime in section 3.2 after discussing when the superthreshold network is synchronizable.

3.1.3. Probability to be cascade-susceptible. The network exhibits synchronous behavior if the firing of one neuron causes all subsequent neurons to fire in immediate succession. To determine if the network is expected to exhibit synchronous behavior, we calculate the probability of all subsequent neurons to fire. We recall from the beginning of section 3 that the network is classified as $p$-synchronizable if the probability of neuronal voltages to be cascade-susceptible is greater than $p$ when the first neuron fires. We will use $p=0.85$ and refer simply to synchronizable networks in the discussion to follow. Many networks that are not synchronizable under this definition might be considered synchronous in some other broader definition. We will work here with this stringent definition, and leave a broader consideration of synchrony for future work.

The probability that the neuronal voltages in the network (2.1), (2.3) are cascadesusceptible is computed by determining if a total firing event will occur given one neuronal voltage is at threshold voltage. A total firing event occurs if the total network coupling input from neurons firing previously in the cascade-firing event with greater voltage is sufficiently large to bring another neuronal voltage above threshold, and therefore fire, perpetuating the cascading event until all neurons fire. At the time the first neuron fires, denoted by $T^{(1)}$, the neuron with the maximum voltage is at threshold, $V^{(N)}=V_{T}$, and we define the remaining ordered neuronal voltages, $V^{(k)}$, where $V^{(k)}>V^{(j)}$ for $k>j$, and write the cascade-susceptible condition for the $k^{\text {th }}$ neuron to fire as

$$
C_{k}: V_{T}-V^{(k)} \leq(N-k) \frac{S}{N}, \quad k \in\{1,2, \ldots N-1\},
$$

where $S$ is the coupling strength between the $N$ neurons in the network. The total firing event occurs if condition (3.25) holds for all $k$, which we write as the cascadesusceptible condition

$$
C=\cap_{k=1}^{N-1} C_{k}: V_{T}-V^{(k)} \leq(N-k) \frac{S}{N}, \quad \forall k \in\{1,2, \ldots N-1\} .
$$

The probability of the event $C$ is computed in terms of the distribution of the remaining neuronal voltages. We will compute this by first conditioning on the time 

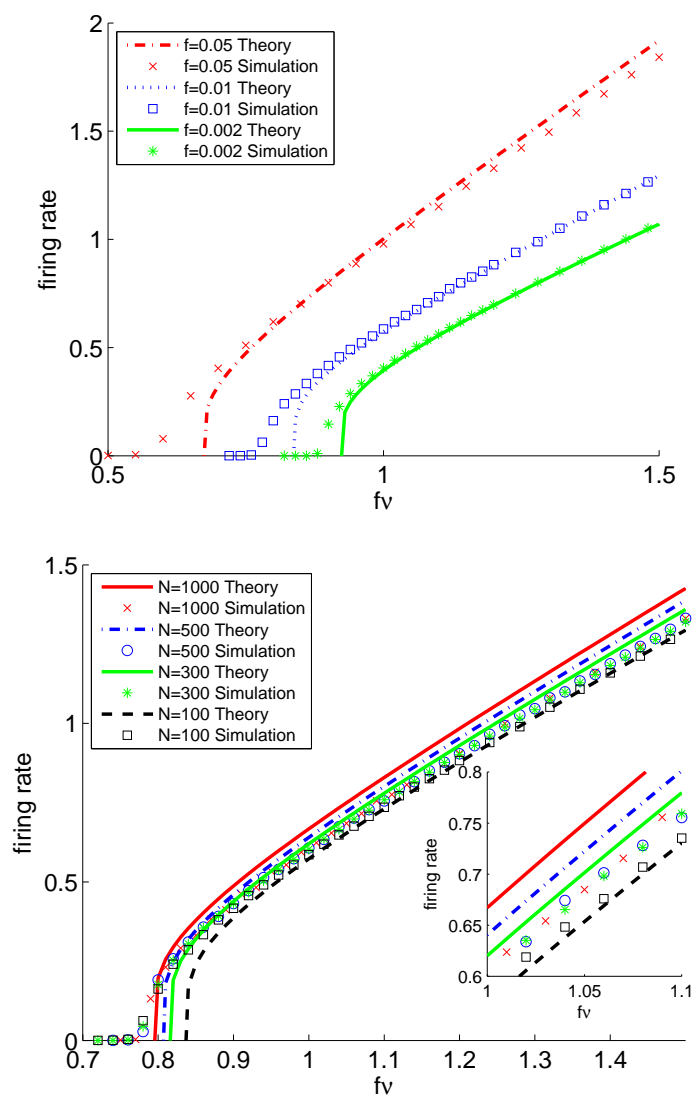

FIG. 3.3. Gain curves - firing rate vs. average external current, f $\nu$,-where equation (3.24) is used to determine the firing rate theoretically, which is then compared to measurements obtained from numerical simulation of the network. (Upper) Effect of the noise strengths: increasing the strength of the noise, which is proportional to $f$, increases the firing rate of the network. (Lower) Effect of the network size: there is a weak dependence on the size of the network. Insert: Magnification of portion of the gain curves. Unless otherwise indicated, the values $N=100, f=0.01$ and $S=2.0$ are used.

that the first neuronal voltage crosses threshold. Then we consider the complement of event (3.26), $C^{c}$, and compute this in terms of the probability of the remaining $N-1$ neurons being distributed in such a way that condition (3.25) fails first at a given value of $k$, and sum over these probabilities.

We compute the probability of condition (3.25) by applying the law of total expectation with respect to the random time $T^{(1)}$ at which the first neuron fires:

$$
P(C)=\int_{0}^{\infty} P\left(C \mid T^{(1)}=t\right) p_{T}^{(1)}(t) d t .
$$

The PDF for the exit time of the first of $N$ neurons, $p_{T}^{(1)}(t)$, is derived from the $\mathrm{PDF}$ for the exit time of a single neuron, $p_{T}(t)$, which in turn is computed from the voltage PDF for a single neuron. If the voltage is allowed to evolve without reset, then integrating the voltage distribution, $p_{v}(x, t)$, over the domain $\left[V_{R}, V_{T}\right]$ results in 


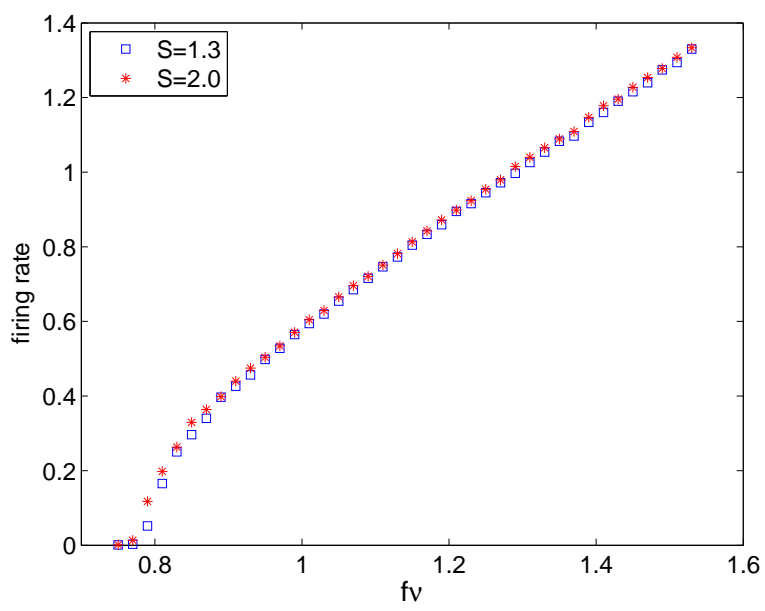

FIG. 3.4. Two gain curves corresponding to the same values of $N=100$ and $f=0.01$, and two different values of the network coupling strength, $S$. The results indicate that the gain curve shape is independent of $S$.

the probability the voltage has not crossed threshold, $P(T<t)$, with the assumption that once the voltage crosses threshold it does not re-cross to be found below at a later time. We have found this assumption to be approximately valid well into the superthreshold regime. The PDF, $p_{v}(x, t)$, is taken to be the Gaussian distribution, equation (3.18), derived in section 3.1.1.

For the conditional probability in equation (3.27) we will consider

$$
P\left(C \mid T^{(1)}=t\right)=1-P\left(C^{c} \mid T^{(1)}=t\right),
$$

the latter being easier to compute explicitly. We simplify the computation of $P\left(C^{c} \mid T^{(1)}=t\right)$ by approximating the probability distribution of the neuronal voltages at the conditioned firing time of the first neuron, $T^{(1)}=t$, as if they were independent and identically distributed random variables, each with PDF $p_{v}(x, t)$ corresponding to uncoupled evolution under equation (2.1) without firing or resetting, but conditioned upon the event that the maximum neuronal voltage is at threshold: $V^{(N)}=V_{T}$. We denote this idealized probability measure as $P_{t \mid V^{(N)}}$, so our approximation reads

$$
P\left(C^{c} \mid T^{(1)}=t\right) \approx P_{t \mid V^{(N)}}\left(C^{c}\right) .
$$

This approximation relies on the probability distribution of the dynamical event $T^{(1)}=t$ being largely determined by the single-time PDFs for the neuronal voltages without firing and resetting, which is plausible for the superthreshold regime where the non-resetting neuronal voltages under the dynamics (2.1) are unlikely to recross threshold at substantially different times. On the other hand the process by which a neuron crosses threshold in the subthreshold regime clearly requires consideration of the dynamical development of a substantial fluctuation in the neuronal voltages, and we have verified that approximation (3.28) does not give very good results. Consequently, here we will only pursue the theoretical estimation of synchronizability for the superthreshold regime. 
The event $C^{c}$ contains at least one value of $k$ for which condition (3.25) fails. At this value of $k$, the previous neurons firing in the cascading event do not increase the voltage $V^{(k)}$ sufficiently to bring it above threshold. The cascading chain is broken and none of the remaining neurons fire. The event $C^{c}$ is partitioned into the mutually exclusive events $\left\{A_{j}\right\}_{j=1}^{N-1}$, where the event $A_{j}$ consists of the arrangements of the $N-1$ neuronal voltages such that condition (3.25) is satisfied for

$k=\{N-1, N-2 \ldots N-j+1\}$ and fails for $k=N-j$. The event $C^{c}$ is the disjoint union of the events $\left\{A_{j}\right\}_{j=1}^{N-1}$, and

$$
P_{t \mid V^{(N)}}\left(C^{c}\right)=\sum_{j=1}^{N-1} P_{t \mid V^{(N)}}\left(A_{j}\right) .
$$

The probability that the distribution of neuronal voltages is cascade-susceptible is reduced to determining the probability of neuronal voltage arrangements.

We evaluate the probabilities $P_{t \mid V^{(N)}}\left(A_{j}\right)$ by dividing up the interval of voltage $\left[V_{R}, V_{T}\right]$ into bins of size $S / N$ and defining the sets

$$
B_{k}=\left\{x: V_{T}-\frac{(k-1) S}{N}>x \geq V_{T}-\frac{k S}{N}\right\} \quad \text { for } k \geq 1
$$

of which there are $b=\left\lceil\left(V_{T}-V_{R}\right) N / S\right\rceil$ in total. Under our approximating probability measure $P_{T \mid V^{(N)}}$, each of the $N-1$ (unordered) voltages which are not at threshold are independent of each other and are distributed according to identical conditioned probability distributions

$$
p_{v \mid V^{(N)}}(x, t)= \begin{cases}\frac{p_{v}(x, t)}{\int_{V_{R}}^{V_{T}} p_{v}\left(x^{\prime}, t\right) \mathrm{d} x^{\prime}} & \text { for } x \in\left[V_{R}, V_{T}\right], \\ 0 & \text { otherwise. }\end{cases}
$$

For the small-fluctuation regime (on which we will focus), we use the Gaussian approximation (3.18) for $p_{v}(x, t)$. Now, under our approximate probability measure, $P_{t \mid V^{(N)}}$, the probability, $p_{j}(t)$, for the voltage of any given neuron to be in bin $B_{j}$ at time $t$ is computed from the integral

$$
p_{j}(t)=\int_{V_{T}-j S / N}^{V_{T}-(j-1) S / N} p_{v \mid V^{(N)}}(x, t) d x \quad \text { for } 1 \leq j \leq b .
$$

We have now set up the framework to determine the probabilities of the events $A_{j}$ under the approximation of $p_{j}(t)$ in equation (3.31). The event $A_{1}$ consists of condition (3.25) failing for $k=N-1$. That is, no neuronal voltages are in the interval $\left(V_{T}-S / N, V_{T}\right]=B_{1}$, thus

$$
P_{t \mid V^{(N)}}\left(A_{1}\right)=P_{t \mid V^{(N)}}\left(v_{1} \ldots v_{N-1} \in B_{1}^{c}\right)=\left(1-p_{1}(t)\right)^{N-1},
$$

using the fact that the (unordered) neuronal voltages are independent. This calculation is extended to the other events $A_{j}, j>1$,

$$
\begin{aligned}
P_{t \mid V^{(N)}}\left(A_{j}\right)= & P_{t \mid V^{(N)}}\left(\left\{V^{(N-1)} \in B_{1}\right\},\left\{V^{(N-1)}, V^{(N-2)} \in \cup_{i=1}^{2} B_{i}\right\}, \ldots,\right. \\
& \left\{V^{(N-1)} \ldots V^{(N-j+1)} \in \cup_{i=1}^{j-1} B_{i}\right\}, \\
& \left.\left\{V^{(N-j)} \ldots V^{(1)} \in\left(\cup_{i=1}^{j} B_{i}\right)^{c}\right\}\right) .
\end{aligned}
$$


The first $j-1$ terms in equation (3.33) describe the arrangement of $j-1$ of the total $N-1$ neuronal voltages such that condition (3.25) is satisfied for $k=N-j+$ $1, \ldots, N-1$. The last term indicates that the cascading event fails after the first $j-1$ neurons fire.

The calculation in equation (3.33) follows from combinatorics if we re-express the events in terms of the unordered independent neuronal voltages. We sum over all configurations of the neuronal voltages consistent with the description of event $A_{j}$, resulting in

$$
\begin{aligned}
P_{t \mid V^{(N)}}\left(A_{j}\right)= & \sum \frac{(N-1) !}{n_{1} ! n_{2} ! \ldots n_{j-1} !(N-j) !} \\
& \times\left(p_{1}(t)\right)^{n_{1}}\left(p_{2}(t)\right)^{n_{2}} \ldots\left(p_{j-1}(t)\right)^{n_{j-1}}\left(\sum_{i=j+1}^{b} p_{i}(t)\right)^{N-j},
\end{aligned}
$$

where $n_{j}$ denotes the number of neurons with voltage in the set $B_{j}$ and $p_{j}$ is defined in equation (3.31).

The probability of the event $C$ with our underlying assumptions is,

$$
P(C)=1-\int_{0}^{\infty} \sum_{j=1}^{N-1} P_{t \mid V^{(N)}}\left(A_{j}\right) p_{T}^{(1)}(t) d t,
$$

which is evaluated numerically. As we now show, the sum can be approximated by only the first few terms. We recall that $P_{t \mid V^{(N)}}\left(A_{j}\right)$ computes the probability that condition (3.25) fails for $k=N-j$. At larger values of $j$, the condition is less likely to fail since the distribution of neuronal voltage is approximately Gaussian with mean close to $V_{T}$. Neurons are more likely to be found closer to the center of the distribution, thus condition (3.26) is more likely to be satsified for smaller values of $k=N-j$, and therefore the failure events, $A_{j}$, become less probable for larger $j$. When evaluating the probability $P(C)$, the series is terminated when terms are less than $10^{-4}$. With this tolerance, 2 to 9 terms are included depending on the parameter values. In figure 3.5 , we compare our theoretical prediction of $P(C)$ from (3.35) with its measurement from direct simulations in which we record the fraction of times the first neuron firing initiates a total firing event when all neuronal voltages begin at $V_{R}$.

The transition from the non synchronizable $(P(C)<0.85)$ network to the synchronizable $(P(C) \geq 0.85)$ network is smooth; see figure 3.5. This transition is sharper for smaller $f$ with $f \nu$ held constant. Larger values of $f$ cause the distribution of neuronal voltages to be more spread out, thus making it less probable that condition (3.26) holds for all values of $k$. Larger values of $f$ correspond to larger fluctuations spreading the neuronal voltages apart, requiring larger network coupling strengths for the cascade-susceptible condition (3.26) to hold with high probability.

We have analyzed a mechanism for the network to maintain a self-consistent state of synchrony and have developed a theoretical characterization of the synchronizability of a network in terms of its governing parameters. Our theoretical prediction of synchronizability through equation (3.35) agrees well with measurements from numerical simulations of the network. One such example appears in figure 3.5.

Over a broad range of parameter values, the network exhibits a large degree of what we would intuitively characterize as synchrony (see figure 2.1 (a) and (b)), but not through strict total firing events. Even though such networks are not "synchronizable" according to our strict definition, our proposed theories for firing rates 


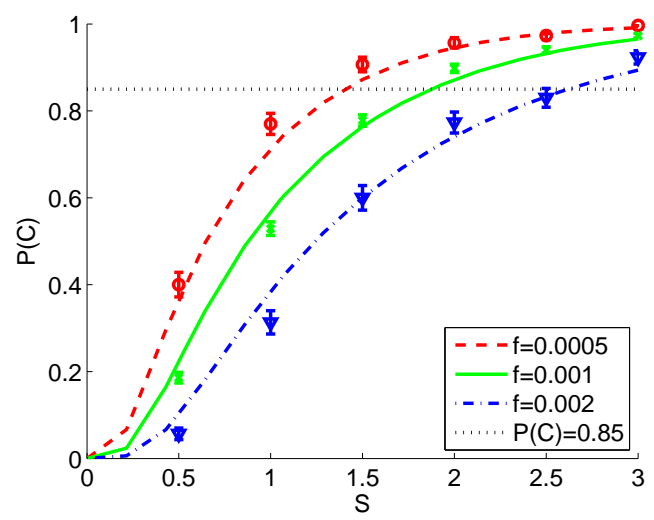

FIG. 3.5. Probability that condition (3.26) holds, $P(C)$, vs. network coupling strength, $S$, for a network with $N=100$ neurons, $f \nu=1.2$, and various values of $f$. Lines - theoretical computation, symbols with arrow bars - Monte Carlo simulations. The transition to synchronizablility (above dashed line at $P(C)=0.85$ ) as $S$ increases is sharper for smaller $f$.

(equations (3.24) and (3.36)) are reasonably good as a few neurons firing slightly early or late relative to a substantial firing cascade do not inject significant error to our calculations performed under the idealized assumption that they fire in total synchrony. Therefore we consider networks with coupling strength $S=2.0$ and values for $f$ and $\nu$ that exhibit effective synchronization properties as observed in our simulations of the full network dynamics, as opposed to limiting ourselves to those that satisfy our strict definition of synchronizability.

3.2. Subthreshold regime. In the completely synchronous network, (2.1) and (2.3), of current-based, I\&F neurons with delta function current impulses, the firing rate is determined entirely by the time between total firing events. After such an event where all neurons fire at the exact same instant, the neuronal voltages are all reset and the process repeats. In the preceding section, we have addressed such total firing events in the superthreshold driving case, in which the neurons are all driven to fire on average.

The method used in section 3.1 is a good approximation when, under the dynamics (3.1) where neurons are uncoupled from each other and their voltages do not reset upon crossing threshold voltage, $V_{T}$, the probability of a neuronal voltage to be below $V_{T}$ given that it was previously above $V_{T}$ is small. A superthreshold driving force (without resetting voltages) will on average push the neuronal voltages quickly past $V_{T}$, so it is unlikely for any neuronal voltage to recross the threshold at substantially different times. When the driving force is subthreshold, a neuronal voltage is always very likely to be below $V_{T}$. This does not imply the neuronal voltage has not crossed $V_{T}$, as there is significant probability that there will be fluctuations in the driving force which push the neuronal voltage just over $V_{T}$. In section 3.1 it was assumed that a neuronal voltage below threshold had not crossed $V_{T}$ at an earlier time, and under the full dynamics had not fired. Therefore, in the subthreshold regime, this approximation cannot be used to accurately compute the average exit time of the first neuron.

In the subthreshold regime, the neuronal voltages lie in wait below threshold for 
a sufficiently large fluctuation to push one neuronal voltage over threshold. For the cascade-susceptible network, this leads to a total firing event. In our superthreshold regime calculations in section 3.1, we developed a satisfactory theory based on the analytical approximation (3.18) of the PDF for the voltage of a single neuron without taking into account its resetting at threshold. This calculation hinged on the assumption that a neuron which crosses threshold without resetting is unlikely to be found later below threshold. While this is a plausible approximation far enough into the superthreshold regime, it is certainly not appropriate for the subthreshold regime. Consequently, we instead obtain the firing rate of the network from the inverse of the expected time for the first of $N$ neurons to fire as computed through a proper first exit time problem. While this computation can again be reduced to a first exit time problem for a single uncoupled neuron, the PDF for this exit time will no longer be computable in terms of the solution of a simple equation with analytical expressions as in equation (3.24) and the surrounding discussion. Rather, this PDF is obtained from the solution of a Kolmogorov forward equation (KFE) with appropriate boundary conditions, expressed in terms of an eigenfunction expansion involving confluent hypergeometric functions.

3.2.1. Exit time relation to PDF for voltage. We consider the inverse of the mean firing rate of the completely synchronous network in the subthreshold regime to be the expectation of the time it takes the first of the $N$ neurons to exit the voltage interval $\left[V_{R}, V_{T}\right]$, i.e., to reach the firing threshold, $V_{T}$. This expectation is computed in terms of the PDF of the exit time for one neuron, in contrast to the PDF of the voltage of a single neuron, which was used in section 3.1. The expected time is given by

$$
\left\langle T^{(1)}\right\rangle=\int_{0}^{\infty} t p_{T}^{(1)}(t) d t
$$

where $p_{T}^{(1)}(t)$ is the PDF of the first exit time, $T^{(1)}$, of the $N$ neurons. The first exit time, $T^{(1)}$, is the minimum of the set of all exit times of $N$ uncoupled neurons. In the beginning of section 3, we pointed out that between total firing events the neurons behave as though they are effectively uncoupled.

The PDF of the minimum exit time, $T^{(1)}$, is calculated from the PDF of one neuron's exit time, $T$, by considering its CDF,

$$
F_{T}^{(1)}(t)=P\left(T^{(1)} \leq t\right)=1-P\left(T^{(1)}>t\right) .
$$

Between total firing events, the $N$ neurons behave independently, thus

$$
P\left(T^{(1)}>t\right)=\left(1-F_{T}(t)\right)^{N},
$$

where $F_{T}(t)$ is the CDF of the exit time for one neuron. Consequently, the PDF of the minimum exit time, $T^{(1)}$, can be related to the PDF, $p_{T}(t)$, of a single neuron's exit time through

$$
p_{T}^{(1)}(t)=N p_{T}(t)\left(1-F_{T}(t)\right)^{N-1},
$$

where $F_{T}(t)=\int_{0}^{t} p_{T}\left(t^{\prime}\right) \mathrm{d} t^{\prime}$ is the CDF of the exit time for a single neuron [7]. We next develop the methods for computing the single-neuron exit time distribution, $p_{T}(t)$. 
Consider that upon reaching threshold voltage, the single neuron is removed from the system, meaning that it is absorbed at the boundary, $V_{T}$, rather than being reset back to $V_{R}$ or being allowed to continue to evolve as in section 3.1. Then, the probability that at time $t$ this neuron has not yet fired (it will fire at a later time $T$ ) is the probability that it is still in the domain $\left[V_{R}, V_{T}\right]$. This probability is

$$
P(T \geq t)=\int_{V_{R}}^{V_{T}} p_{v}(x, t) d x,
$$

where $x$ is the neuronal voltage and the PDF, $p_{v}(x, t)$, satisfies the KFE for a single, Poisson spike-train driven, I\&F neuron with an absorbing barrier at $V_{T}[15,27,77,78]$. The KFE satisfied by the PDF, $p_{v}(x, t)$, will be derived in section 3.2.2. Note that the PDF, $p_{v}(x, t)$, is defective in the sense that its integral over the domain $x \in$ $\left[V_{R}, V_{T}\right]$ does not equal one for all times. The difference is exactly the probability that the neuron has reached threshold, $V_{T}$, and therefore been removed from further consideration.

Equation (3.39) can be expressed in terms of the CDF, $F_{T}(t)$, of a single neuron's first exit time as

$$
P(T \geq t)=1-F_{T}(t) .
$$

From this equation, the PDF of this first exit time is derived in the form

$$
p_{T}(t)=-\frac{d}{d t} P(T \geq t)=-\frac{\partial}{\partial t} \int_{V_{R}}^{V_{T}} p_{v}(x, t) d x .
$$

By taking the derivative inside the integral, this is equivalent to

$$
p_{T}(t)=\int_{V_{R}}^{V_{T}}-\frac{\partial}{\partial t} p_{v}(x, t) d x=\int_{V_{R}}^{V_{T}} \frac{\partial}{\partial x} J\left[p_{v}\right](x, t) d x
$$

where the flux,

$$
J\left[p_{v}\right](x, t)=-\left(g_{L}\left(x-V_{R}\right)-f \nu\right) p_{v}(x, t)-\frac{f^{2} \nu}{2} \frac{\partial}{\partial x} p_{v}(x, t),
$$

is obtained from the diffusion approximation of the KFE, to be derived next in section 3.2.2. Equation (3.42) is the flux of probability that leaves through the upper boundary,

$$
p_{T}(t)=J\left[p_{v}\right]\left(V_{T}, t\right),
$$

as no flux leaves through the lower boundary due to the fact that the Poisson driven neuronal voltage never falls below $V_{R}$. The problem of finding the expected time between total firing events is reduced to determining $p_{v}(x, t)$ with the prescribed boundary conditions, and then obtaining the flux at $x=V_{T}$. The KFE that $p_{v}(x, t)$ satisfies is developed next.

3.2.2. Kolmogorov forward and backward equations. The PDF of the voltage for a single neuron, $p_{v}(x, t)$, is the solution to the KFE with an absorbing boundary at $x=V_{T}$. This equation may be thought of as expressing the conservation of probability density, and reads

$$
\frac{\partial}{\partial t} p_{v}(x, t)=\frac{\partial}{\partial x}\left[g_{L}\left(x-V_{R}\right) p_{v}(x, t)\right]+\nu\left[p_{v}(x-f, t)-p_{v}(x, t)\right]
$$


where incoming spikes are modeled by a Poisson spike train with rate $\nu$ and strength $f$.

The rigorous derivation of this equation is best accomplished by first working with the probability transition density, $p_{v}(y, s ; x, t)$, defined so that

$$
P\left(v_{j}(t) \in B \mid v_{j}(s)=y\right)=\int_{B} p_{v}(y, s ; x, t) \mathrm{d} x
$$

for any nice (Borel) subset $B$ of the real line and pair of times $s<t$, where $v_{j}(t)$ is the solution of the differential equation for the voltage (3.1). As can be seen from this definition, the dependence of the probability transition density $p_{v}(y, s ; x, t)$ on $x$ is as a density variable (representing the Radon-Nikodym derivative of a probability measure), whereas its dependence on $y$ is as straightforward initial data. This is why the Kolmogorov backward equation (KBE, expressed in terms of the source variables $s$ and $y$ ) is easier to develop than the KFE (expressed in terms of the target variables $t$ and $x$ ). Once the KBE is obtained, the KFE follows directly through an adjoint operation [59].

Our strategy will be to relate the derivation of the backward evolution equation for the probability transition density to that corresponding to a differential equation for $u_{j}$, the neuronal voltages in the network without the Poisson spiking terms:

$$
\frac{d u_{j}}{d t}=-g_{L}\left(u_{j}-V_{R}\right)
$$

The KBE associated to this equation is, from the method of characteristics, simply

$$
\begin{aligned}
& -\frac{\partial p_{u}(y, s ; x, t)}{\partial s}=-g_{L}\left(y-V_{R}\right) \frac{\partial p_{u}(y, s ; x, t)}{\partial y} \\
& p_{u}(y, s=t ; x, t)=\delta(x-y),
\end{aligned}
$$

(to be solved backward in $s$ from $s=t$ into $s<t$ ).

Returning to the actual dynamics for $v_{j}(t)$, we follow the standard technique of invoking the Chapman-Kolmogorov Equation [44, Sec. 4.4a] to express the probability transition density under a small time change, $\Delta s$, in the source time argument:

$$
p(y, s-\Delta s ; x, t)=\int_{-\infty}^{\infty} p(y, s-\Delta s ; z, s) p(z, s ; x, t) \mathrm{d} z .
$$

We now work on the first factor in the integrand by integrating its target variable over an arbitrary open set $B$ so we can directly deal with a well-defined probability:

$$
P\left(v_{j}(s) \in B \mid v_{j}(s-\Delta s)=y\right)=\int_{B} p(y, s-\Delta s ; z, s) \mathrm{d} z .
$$

Following the familiar practice in continuous-time Markov chains [44, Sec. 4.5], we decompose this probability with respect to a partition corresponding to the number of spiking events, $M(s)-M(s-\Delta s)$, (where $M(s)$ is a Poisson counting process [44, Sec. $1.3 \mathrm{f}]$ with rate $\nu$ describing the number of spiking events on the interval $[0, s])$ that 
occur during the small time interval $(s-\Delta s, s]$ :

$$
\begin{aligned}
& P\left(v_{j}(s) \in B \mid v_{j}(s-\Delta s)=y\right) \\
= & P\left(v_{j}(s) \in B \mid v_{j}(s-\Delta s)=y, M(s)-M(s-\Delta s)=0\right) \\
& \times P\left(M(s)-M(s-\Delta s)=0 \mid v_{j}(s-\Delta s)=y\right) \\
& +P\left(v_{j}(s) \in B \mid v_{j}(s-\Delta s)=y, M(s)-M(s-\Delta s)=1\right) \\
& \times P\left(M(s)-M(s-\Delta s)=1 \mid v_{j}(s-\Delta s)=y\right) \\
& +P\left(v_{j}(s) \in B \mid v_{j}(s-\Delta s)=y, M(s)-M(s-\Delta s) \geq 2\right) \\
& \times P\left(M(s)-M(s-\Delta s) \geq 2 \mid v_{j}(s-\Delta s)=y\right) .
\end{aligned}
$$

The number of spiking events on the interval $(s-\Delta s, s]$ obeys a Poisson distribution with mean $\nu \Delta s$ and is independent of the voltage at the beginning of the time interval:

$$
\begin{aligned}
P\left(M(s)-M(s-\Delta s)=m \mid v_{j}(s-\Delta s)=y\right) & =P(M(s)-M(s-\Delta s)=m) \\
& =\frac{(\nu \Delta s)^{m}}{m !} \mathrm{e}^{-\nu \Delta s},
\end{aligned}
$$

so in particular for small $\Delta s$ :

$$
\begin{aligned}
& P\left(M(s)-M(s-\Delta s)=0 \mid v_{j}(s-\Delta s)=y\right)=1-\nu \Delta s+O\left((\Delta s)^{2}\right), \\
& P\left(M(s)-M(s-\Delta s)=1 \mid v_{j}(s-\Delta s)=y\right)=\nu \Delta s+O\left((\Delta s)^{2}\right), \\
& P\left(M(s)-M(s-\Delta s) \geq 2 \mid v_{j}(s-\Delta s)=y\right)=O\left((\Delta s)^{2}\right) .
\end{aligned}
$$

On the other hand,

$$
\begin{aligned}
P\left(v_{j}(s) \in B \mid v_{j}(s-\Delta s)\right. & =y, M(s)-M(s-\Delta s)=0) \\
& =P\left(u_{j}(s) \in B \mid v_{j}(s-\Delta s)=y, M(s)-M(s-\Delta s)=0\right) \\
& =P\left(u_{j}(s) \in B \mid u_{j}(s-\Delta s)=y\right)
\end{aligned}
$$

and

$$
\begin{aligned}
P\left(v_{j}(s) \in B \mid v_{j}(s-\Delta s)\right. & =y, M(s)-M(s-\Delta s)=1) \\
& =P\left(u_{j}(s)+f+e_{1}(\Delta s) \in B \mid u_{j}(s-\Delta s)=y\right) \\
& =P\left(u_{j}(s)+e_{2}(\Delta s) \in B \mid u_{j}(s-\Delta s)=y+f\right),
\end{aligned}
$$

where the $e_{j}(\Delta s)$ are random errors (related to exactly when the spike appears in the time interval $(s-\Delta s, s])$ which vanish in the limit $\Delta s \rightarrow 0$ and the $u_{j}$ obey the dynamics of equation (3.46). Therefore, because $B$ is open,

$$
\begin{aligned}
\lim _{\Delta \downarrow \downarrow 0} P\left(v_{j}(s) \in B \mid v_{j}(s-\Delta s)\right. & =y, M(s)-M(s-\Delta s)=1) \\
& =\delta_{y+f}(B) \equiv \begin{cases}1, & \text { if } y+f \in B, \\
0, & \text { otherwise, }\end{cases}
\end{aligned}
$$

and similarly

$$
\lim _{\Delta s \downarrow 0} P\left(u_{j}(s) \in B \mid u_{j}(s-\Delta s)=y\right)=\delta_{y}(B) .
$$


Substituting these expressions into the decomposition (3.49), we obtain

$$
\begin{aligned}
& P\left(v_{j}(s) \in B \mid v_{j}(s-\Delta s)=y\right) \\
= & \left(1-\nu \Delta s+O\left((\Delta s)^{2}\right)\right) P\left(u_{j}(s) \in B \mid u_{j}(s-\Delta s)=y\right) \\
& +\left(\nu \Delta s+O(\Delta s)^{2}\right)\left(\delta_{y+f}(B)+o(1)\right) \\
= & P\left(u_{j}(s) \in B \mid u_{j}(s-\Delta s)=y\right)-(\nu \Delta s) \delta_{y}(B)+\nu \Delta s \delta_{y+f}(B)+o(\Delta s) \\
= & P\left(u_{j}(s) \in B \mid u_{j}(s-\Delta s)=y\right)+(\nu \Delta s)\left(\delta_{y+f}(B)-\delta_{y}(B)\right) .
\end{aligned}
$$

Since $B$ is an arbitrary open set, we can read-off from this an asymptotic approximation for the (generalized) probability transition density

$$
p_{v}(y, s-\Delta s ; z, s)=p_{u}(y, s-\Delta s ; z, s)+(\nu \Delta s)(\delta(z-(y+f))-\delta(z-y))+o(\Delta s) .
$$

This is the key expression which explains the form of the Kolmogorov backward and forward equations for the probability density for the voltages - over a short time interval of width $\Delta s$ the probability transition density evolves just as if there were no spikes (as described by the $u_{j}$ dynamics of equation (3.46)) but with an additional transition from the current voltage value $y$ to $y+f$ when a spike appears (with probability approximately $\nu \Delta s$ ). Events where two or more spikes appear in a short interval have negligibly small probability.

We now complete the derivation in the standard way by using expression (3.50) along with the identity $p_{v}(s, y ; s, x)=p_{u}(s, y ; s, x)=\delta(x-y)$ in the ChapmanKolmogorov equation (3.48):

$$
\begin{aligned}
& -\frac{\partial p_{v}(y, s ; x, t)}{\partial s} \\
= & \lim _{\Delta s \downarrow 0} \frac{p_{v}(y, s-\Delta s ; x, t)-p_{v}(y, s ; x, t)}{\Delta s} \\
= & \lim _{\Delta s \downarrow 0} \int_{-\infty}^{\infty} p_{v}(z, s ; x, t) \frac{p_{v}(y, s-\Delta s ; z, s)-p_{v}(y, s ; z, s)}{\Delta s} \mathrm{~d} z \\
= & \lim _{\Delta s \downarrow 0} \int_{-\infty}^{\infty} p_{v}(z, s ; x, t)\left[\frac{p_{u}(y, s-\Delta s ; z, s)-p_{u}(y, s ; z, s)}{\Delta s}\right. \\
= & \int_{-\infty}^{\infty} p_{v}(z, s ; x, t)\left[-\left.\frac{\partial p_{u}\left(y, s ; z, s^{\prime}\right)}{\partial s}\right|_{s^{\prime}=s}+\nu(\delta(z-(y+f))-\delta(z-y))\right] \mathrm{d} z .
\end{aligned}
$$

Using the evolution equation (3.47) for the probability transition density $p_{u}$, we obtain

$$
\begin{aligned}
& -\frac{\partial p_{v}(y, s ; x, t)}{\partial s} \\
= & \int_{-\infty}^{\infty} p_{v}(z, s ; x, t)\left[-g_{L}\left(y-V_{R}\right) \frac{\partial \delta(z-y)}{\partial y}+\nu(\delta(z-(y+f))-\delta(z-y))\right] \mathrm{d} z,
\end{aligned}
$$

which reduces to the KBE for the probability transition density:

$$
-\frac{\partial p_{v}(y, s ; x, t)}{\partial s}=-g_{L}\left(y-V_{R}\right) \frac{\partial p_{v}(y, s ; x, t)}{\partial y}+\nu\left(p_{v}(y+f, s ; x, t)-p_{v}(y, s ; x, t)\right) .
$$


We used Dirac delta functions in (3.51) to describe the formal structure of the argument; they can be avoided through more cumbersome analysis involving estimates controlling the probability for the underlying stochastic process $v_{j}$ to vary in a way substantially different from that suggested by the Dirac delta functions [59].

Having obtained the KBE for the evolution of the probability transition density with respect to its source variables $s$ and $y$, we can now write down the KFE for the evolution of the probability transition density with respect to target variables $t$ and $x$ through taking the adjoint of the (infinitesmal generator) operator implied by the right hand side of (3.52) and applying it to the $(t, x)$ variables:

$$
\frac{\partial p_{v}(y, s ; x, t)}{\partial t}=\frac{\partial}{\partial x}\left(g_{L}\left(x-V_{R}\right) p_{v}(y, s ; x, t)\right)+\nu\left(p_{v}(y, s ; x-f, t)-p_{v}(y, s ; x, t)\right) .
$$

The KFE (3.45) for the probability density of the voltage itself is simply obtained by observing from the law of total probability that:

$$
p_{v}(x, t)=\int_{-\infty}^{\infty} p_{v}(y, 0 ; x, t) p_{v}(y, 0) d y .
$$

In other words, the probability transition density acts like a Green's function for the probability density, and therefore satisfies the same evolution equation. This concludes our derivation of the Kolmogorov equations, and we proceed to find their approximation solutions in the small-fluctuation regime.

An approximation to the KFE is obtained by considering $f$ small, which is the case for the synchronized network. In this small-fluctuation regime, where $f \nu$ is held constant, $\nu$ is then large. The voltage jumps a small amount for each incoming spike and does not have long to decay before the next spike arrives, thus the voltage approximates a smooth function. By Taylor expanding the function $p_{v}(x-f, t)$ for small $f$, we generate a Kramers-Moyal expansion [75, Sec. 4.1]. Keeping the first three terms in this expansion, equation (3.45) is reduced to the Fokker-Planck form

$$
\frac{\partial}{\partial t} p_{v}(x, t)=\frac{\partial}{\partial x}\left[\left(g_{L}\left(x-V_{R}\right)-f \nu\right) p_{v}(x, t)\right]+\frac{f^{2} \nu}{2} \frac{\partial^{2}}{\partial x^{2}} p_{v}(x, t),
$$

in which the difference term has been replaced by drift and diffusion terms. Equation (3.53) can be written in the conservation form

$$
\frac{\partial}{\partial t} p_{v}(x, t)+\frac{\partial}{\partial x} J\left[p_{v}\right](x, t)=0,
$$

with the probability flux $J\left[p_{v}\right](x, t)$ defined in equation (3.43).

The approximate KFE (3.53) requires that a second boundary condition be imposed. In particular, a reflecting boundary condition is posed at $x=V_{R}$ because the actual neuronal voltage cannot go below threshold, nor are any neurons injected into the network at the voltage value $x=V_{R}$, due to the fact that firing neurons are removed from the system rather than having their voltages reset to $V_{R}$, as discussed above. This reflecting boundary condition requires that the probability flux $J\left[p_{v}\right](x, t)$ must vanish at $x=V_{R}$.

The initial condition for equation (3.53) is

$$
p_{v}(x, 0)=\delta\left(x-V_{R}\right),
$$


as the neuronal voltage always starts at the reversal potential after a total firing event. From equation (3.43), we see that the reflecting boundary condition at $V_{R}$ is

$$
\left.\frac{f^{2} \nu}{2} \frac{\partial}{\partial x} p_{v}(x, t)\right|_{x=V_{R}}-f \nu p_{v}\left(V_{R}, t\right)=J\left[p_{v}\right]\left(V_{R}, t\right)=0,
$$

and the absorbing boundary condition at $V_{T}$ is

$$
p_{v}\left(V_{T}, t\right)=0
$$

Equation (3.53) along with initial condition (3.55) and boundary conditions (3.56) is the approximate KFE. The corresponding KBE will be needed to construct the solution to the KFE. As the adjoint of the KFE, integration by parts yields this KBE:

$$
\frac{\partial}{\partial t} q(x, t)=\left[-g_{L}\left(x-V_{R}\right)+f \nu\right] \frac{\partial}{\partial x} q(x, t)+\frac{f^{2} \nu}{2} \frac{\partial^{2}}{\partial x^{2}} q(x, t)
$$

with the adjoint boundary conditions

$$
\left.\frac{\partial}{\partial x} q(x, t)\right|_{x=V_{R}}=0
$$

and

$$
q\left(V_{T}, t\right)=0 .
$$

The method for obtaining an eigenfunction expansion of the analytic solution $p_{v}(x, t)$ to the KFE will be outlined next. While equation (3.53) could also be solved directly with an appropriate numerical method, the eigenfunction expansion avoids accumulation of error from time integration and allows precise computation of derivatives involved in the boundary flux (3.44); see appendix C.3.

3.2.3. Solving the approximate KFE. To solve equation (3.53), we consider a separation of variables, and a series expansion of the form

$$
p_{v}(x, t)=\sum_{n=0}^{\infty} A_{n} P_{n}(x) e^{-\lambda_{n} t}
$$

where the $n^{\text {th }}$ eigenfunction, $P_{n}(x)$, solves the eigenvalue problem

$$
\frac{d}{d x}\left[\left(g_{L}\left(x-V_{R}\right)-f \nu\right) P_{n}(x)\right]+\frac{f^{2} \nu}{2} \frac{d^{2}}{d x^{2}} P_{n}(x)+\lambda_{n} P_{n}(x)=0 .
$$

The boundary conditions of this eigenvalue problem are

$$
\left.\frac{f^{2} \nu}{2} \frac{d}{d x} P_{n}(x)\right|_{x=V_{R}}-f \nu P_{n}\left(V_{R}\right)=0
$$

and

$$
P_{n}\left(V_{T}\right)=0
$$

in accordance with the homogenous boundary conditions (3.56) for the full KFE. 
The operator in the eigenvalue problem (3.59) is not symmetric, which normally would require solving both the direct and the adjoint problem in order to find biorthogonal sets of eigenfunctions that would allow us to determine the terms of the series in equation (3.58). We obviate this in appendix B by transforming the equation into a self-adjoint form. Also in appendix B we demonstrate the property

$$
p_{v}(x, t)=p_{s}(x) q(x, t)
$$

holds for our boundary conditions (3.56) for the full KFE, where $q(x, t)$ solves the adjoint problem $(\mathrm{KBE}(3.57))$ and $p_{s}(x)$ is the stationary distribution for the related problem of equation (3.53) but with reflecting boundary conditions at both ends (zero flux). The stationary solution is derived in appendix A to be

$$
p_{s}(x)=\mathcal{N} \exp \left(-\frac{\left(g_{L}\left(x-V_{R}\right)-f \nu\right)^{2}}{f^{2} \nu g_{L}}\right)
$$

where the constant $\mathcal{N}$ would normally be chosen so that $p_{s}(x)$ integrates to one over the domain. However, our use of the stationary distribution to transform the equations for the eigenfunctions $P_{n}(x)$ does not rely on it being properly normalized, so we choose $\mathcal{N}=1$ for simplicity.

It is now justified to write the PDF of the voltage in the form

$$
p_{v}(x, t)=p_{s}(x) \sum_{n=0}^{\infty} A_{n} Q_{n}(x) e^{-\lambda_{n} t}
$$

where $Q_{n}(x)$ and $\lambda_{n}$ are the eigenfunctions and eigenvalues of the problem

$$
\left[-g_{L}\left(x-V_{R}\right)+f \nu\right] \frac{d}{d x} Q_{n}(x)+\frac{f^{2} \nu}{2} \frac{d^{2}}{d x^{2}} Q_{n}(x)+\lambda_{n} Q_{n}(x)=0,
$$

with boundary conditions

$$
\left.\frac{d}{d x} Q_{n}(x)\right|_{x=V_{R}}=0
$$

and

$$
Q_{n}\left(V_{T}\right)=0 .
$$

The constants $A_{n}$ are determined from the initial condition (3.55) via the equation

$$
\int_{V_{R}}^{V_{T}} \delta\left(x-V_{R}\right) Q_{n}(x) d x=A_{n} \int_{V_{R}}^{V_{T}} p_{s}(x) Q_{n}^{2}(x) d x
$$

for which

$$
A_{n}=\frac{Q_{n}\left(V_{R}\right)}{\int_{V_{R}}^{V_{T}} p_{s}(x) Q_{n}^{2}(x) d x} .
$$

The solution to equation (3.63) is obtained through two transformations of variables. First, we shift and rescale the variable $x$ so that

$$
z=\frac{g_{L}\left(x-V_{R}\right)-f \nu}{f \sqrt{g_{L} \nu}} .
$$


The system is driven to $z=0$ by the average drift, and $x$ is essentially rescaled by the standard deviation, which is $O(f \sqrt{\nu})$. The eigenfunction equation (3.63) for $Q_{n}(x)$ under this transformation becomes the equation for $\tilde{Q}_{n}(z)$

$$
\tilde{Q}_{n}^{\prime \prime}(z)-2 z \tilde{Q}_{n}^{\prime}(z)+\frac{2 \lambda_{n}}{g_{L}} \tilde{Q}_{n}(z)=0
$$

in which $Q_{n}(x)=\tilde{Q}_{n}(z(x))$. This would be Hermite's equation if $2 \lambda / g_{L}$ happened to be an integer. Taking another transformation, $\zeta=z^{2}$, the above equation for $\tilde{Q}_{n}(z)$ becomes the equation for $\hat{Q}_{n}(\zeta)$

$$
\zeta \hat{Q}_{n}^{\prime \prime}(\zeta)+\left(\frac{1}{2}-\zeta\right) \hat{Q}_{n}^{\prime}(\zeta)+\frac{\lambda_{n}}{2 g_{L}} \hat{Q}_{n}(\zeta)=0,
$$

where again, $Q_{n}(x)=\hat{Q}_{n}(\zeta(z(x)))$.

Equation (3.68) is a differential equation of the form

$$
\xi \frac{d^{2} w(\xi)}{d \xi^{2}}+(b-\xi) \frac{d w(\xi)}{d \xi}-a w(\xi)=0,
$$

whose solution is the confluent hypergeometric function [2], also known as Kummer's Function,

$$
{ }_{1} F_{1}(a, b, \xi)=\sum_{n=0}^{\infty} \frac{(a)_{n} \xi^{n}}{(b)_{n} n !}
$$

where

$$
(c)_{n}=c(c+1)(c+2) \ldots(c+n-1), \quad(c)_{0}=1
$$

is the Pochhammer symbol [83]. The second linearly independent solution will be chosen either as

$$
U(a, b, \xi)=\frac{\pi}{\sin \pi b}\left[\frac{{ }_{1} F_{1}(b-a, b, \xi)}{\Gamma(1+a-b) \Gamma(b)}-\frac{\xi^{1-b}{ }_{1} F_{1}(1-a, 2-b, \xi)}{\Gamma(a) \Gamma(2-b)}\right]
$$

or as

$$
\xi^{1-b}{ }_{1} F_{1}(1-a, 2-b, \xi) .
$$

The choice of which two linearly independent solutions to use is discussed in appendix C.1. For our equation (3.67), the three solutions are

$$
{ }_{1} F_{1}\left(-\frac{\lambda_{n}}{2 g_{L}}, \frac{1}{2}, z^{2}\right), \quad U\left(-\frac{\lambda_{n}}{2 g_{L}}, \frac{1}{2}, z^{2}\right), \text { and, }|z|_{1} F_{1}\left(-\frac{\lambda_{n}}{2 g_{L}}+\frac{1}{2}, \frac{3}{2}, z^{2}\right),
$$

where the replacement $\zeta=z^{2}$ has been made.

The second and third functions in (3.74) are not smooth at $z=0$, since the mapping of $z \rightarrow \zeta$ is $2-1$, rather than 1-1. As the solutions of (3.67) must be continuously differentiable at $z=0$, two different linear combinations must be taken for $z>0$ and $z<0$, with function and derivative matching at $z=0$. For any given basis of $y_{1}(z)$ and $y_{2}(z)$, the solution is written as

$$
\tilde{Q}_{n}(z)= \begin{cases}c_{1} y_{1}(z)+c_{2} y_{2}(z) & \text { for } \quad z \leq 0 \\ c_{3} y_{1}(z)+c_{4} y_{2}(z) & \text { for } \quad z>0\end{cases}
$$



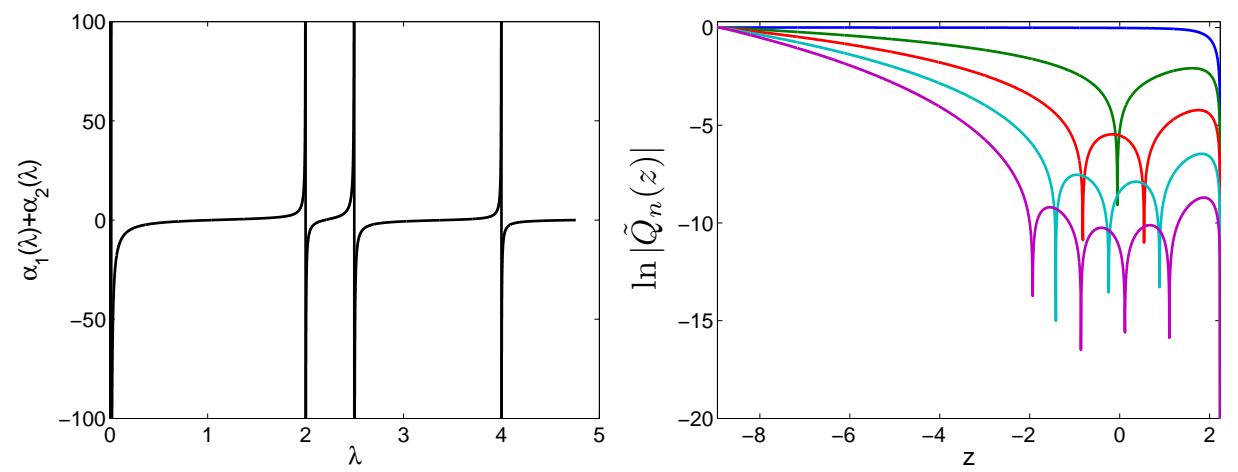

FIG. 3.6. (Left) Equation (3.78) is plotted using the basis ${ }_{1} F_{1}$ and $z_{1} F_{1}$, the zeros of which are the eigenvalues. (Right) Plotted on a log scale with the transformed variable $z$, from $z\left(V_{R}\right)$ to $z\left(V_{T}\right)$, are the absolute values of the first 5 eigenfunctions, $\left|\tilde{Q}_{n}(z)\right|$, of the $K B E$ using basis ${ }_{1} F_{1}$ and $U$ in equation (3.74) for $f=0.01$ and $\nu=80$.

In general, the eigenvalues are determined by requiring the boundary conditions together with the matching conditions at $z=0$ to be met. This gives the following $4 \times 4$ system of equations for $c_{1}, c_{2}, c_{3}$, and $c_{4}$, which must be singular,

$$
\left[\begin{array}{cccc}
\frac{d y_{1}\left(z_{R}\right)}{d z} & \frac{d y_{2}\left(z_{R}\right)}{d z} & 0 & 0 \\
0 & 0 & y_{1}\left(z_{T}\right) & y_{2}\left(z_{T}\right) \\
\lim _{z \rightarrow 0^{-}} y_{1}(z) & \lim _{z \rightarrow 0^{-}} y_{2}(z) & -\lim _{z \rightarrow 0^{+}} y_{1}(z) & -\lim _{z \rightarrow 0^{+}} y_{1}(z) \\
\lim _{z \rightarrow 0^{-}} \frac{d y_{1}}{d z} & \lim _{z \rightarrow 0^{-}} \frac{d y_{2}}{d z} & -\lim _{z \rightarrow 0^{+}} \frac{d y_{1}}{d z} & -\lim _{z \rightarrow 0^{+}} \frac{d y_{2}}{d z}
\end{array}\right]\left[\begin{array}{l}
c_{1} \\
c_{2} \\
c_{3} \\
c_{4}
\end{array}\right]=\left[\begin{array}{l}
0 \\
0 \\
0 \\
0
\end{array}\right]
$$

Rather than take the determinant of this system, it can be simplified and row reduced to a simple equation for the eigenvalues. The choice of $y_{1}(z)={ }_{1} F_{1}\left(-\frac{\lambda_{n}}{2 g_{L}}, \frac{1}{2}, z^{2}\right)$ and $y_{2}(z)=|z|_{1} F_{1}\left(-\frac{\lambda_{n}}{2 g_{L}}+\frac{1}{2}, \frac{3}{2}, z^{2}\right)$ is explained in appendix C.1, which simplifies the system to

$$
\left[\begin{array}{cccc}
1 & 0 & 0 & -\alpha_{1}\left(\lambda_{n}\right) \\
0 & 1 & 0 & 1 \\
0 & 0 & 1 & -\alpha_{1}\left(\lambda_{n}\right) \\
0 & 0 & 0 & \alpha_{1}\left(\lambda_{n}\right)+\alpha_{2}\left(\lambda_{n}\right)
\end{array}\right]\left[\begin{array}{l}
c_{1} \\
c_{2} \\
c_{3} \\
c_{4}
\end{array}\right]=\left[\begin{array}{l}
0 \\
0 \\
0 \\
0
\end{array}\right]
$$

where $\alpha_{1}\left(\lambda_{n}\right)=\frac{d y_{2}\left(z_{R}\right)}{d z} / \frac{d y_{1}\left(z_{R}\right)}{d z}$ and $\alpha_{2}\left(\lambda_{n}\right)=y_{2}\left(z_{T}\right) / y_{1}\left(z_{T}\right)$. The equation of the simple form,

$$
\alpha_{1}\left(\lambda_{n}\right)+\alpha_{2}\left(\lambda_{n}\right)=0,
$$

is solved numerically for the eigenvalues. Equation (3.78) along with the absolute value of the first few eigenfunctions appear in figure 3.6. The stiffness of the problem is evident in the log plot of the eigenfunctions in figure 3.6, which shows that the local and global maxima of their absolute values vary over 10 to 15 orders of magnitude over the finite interval. We address numerically evaluating these eigenfunctions in appendix C. 


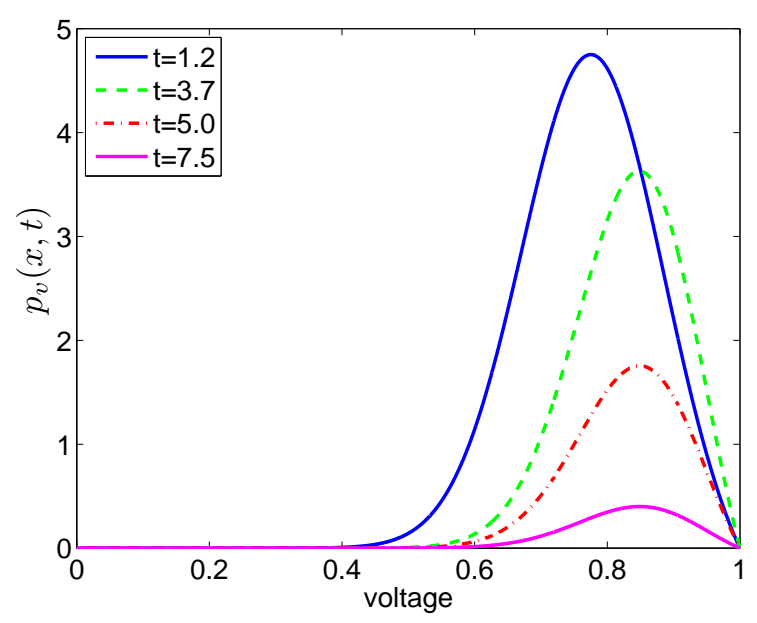

FIG. 3.7. Solutions to the KFE (3.53) for a single neuronal voltage which has one absorbing boundary condition at $V_{T}, f=0.05$, and $f \nu=0.91$ at four times. The integral of $p_{v}(x, t)$ over the domain $[0,1]$ is the probability that the voltage has not yet crossed threshold at time $t$.

The solution, $p_{v}(x, t)$, to equation (3.53) is obtained numerically by first obtaining a finite number of solutions to equation (3.78) for the eigenvalues, $\lambda_{n}$. For each value of $\lambda_{n}$, the corresponding eigenfunction, $Q_{n}(x)$, is evaluated with an appropriate linear combination of the functions in (3.74). The coefficient, $A_{n}$, is determined from equation (3.65) by numerically evaluating the integral. These values are used to compute the terms in (3.62) which are added together to form $p_{v}(x, t)$ (see figure 3.7) and are used to compute the terms in (C.14) and added together to form $p_{T}(t)$ (see figure 3.8). Details on numerically computing the confluent hypergeometric functions appear in appendix C.2. The validity of this solution and the firing rates computed with it are discussed next.

3.2.4. Firing rate. Having outlined the solution procedure to obtain the mean exit time of the first neuron from the network in section 3.2.1 and 3.2.2, we proceed to compare this solution from the approximation of the KFE to measurements obtained from simulations of system (2.1) and (2.3) under the Poisson spike train drive. We compare the PDFs for the first exit time of the $N$ neurons as well as the average firing rate with emphasis on the subthreshold regime, where we use the results of the detailed calculation of the exit time PDF.

The mechanism for synchrony predicts the firing rate of the network to be the inverse of the time the first of $N$ neurons fire on average, $1 /\left\langle T^{(1)}\right\rangle$. We first compare the predicted first exit time PDF, equation (3.38), to that obtained from the simulations of system (2.1), (2.3). These are plotted in figure 3.8 for two different values of $f$. For the larger value of $f$, the diffusion approximation of the KFE is less accurate. The numerically measured exit times are shifted to smaller times, as the discrete jumps in potential cause the voltage to jump above threshold sooner than the voltage under the continuous diffusion approximation. Thus the neuronal voltages driven by Poisson spikes exit sooner, this shift being more noticeable with larger discontinuities (larger values of $f$ ) in the voltage.

The solution to the KFE is used to evaluate equation (3.36) for the average time 


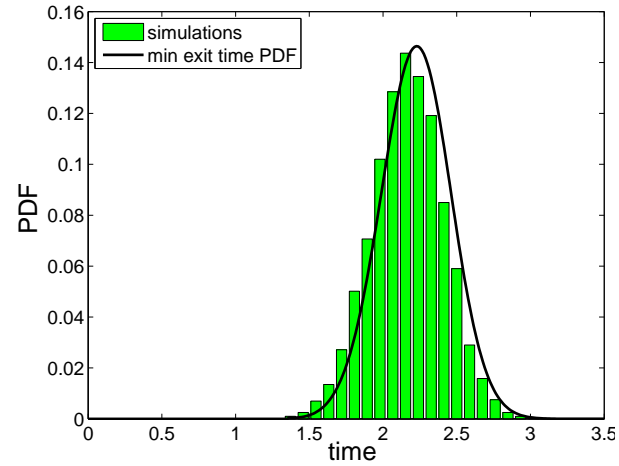

(a) $f=0.01, f \nu=0.91$

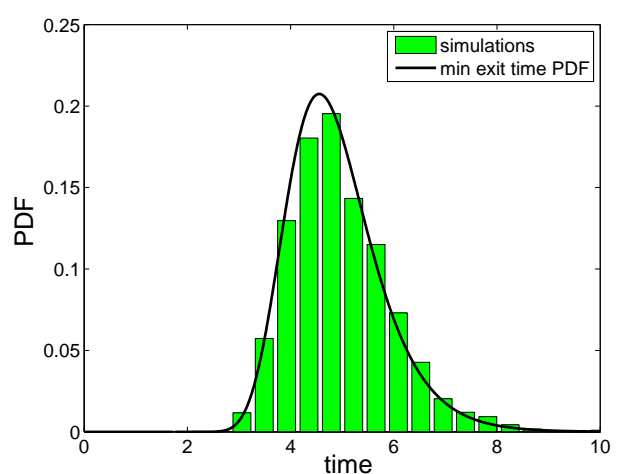

(b) $f=0.002, f \nu=0.91$

FIG. 3.8. Comparison of the predicted distribution for the first exit times $\left(p_{T}^{(1)}(t)\right.$ from equation (3.38)) to those obtained from numerical simulations of networks driven by Poisson point processes ( $N=100$ and coupling strength $S=2.0)$. The figure on the right is well within the small-fluctuation regime, where the diffusion approximation is valid. The figure on the left is the case for which $f$ is relatively large, and the diffusion approximation remains reasonable. However, the Poisson driven neurons exit slightly sooner than the smooth diffusion approximation.

between synchronous firing events. The firing rate, computed as the inverse of this average time, is in good agreement with the firing rate measured from simulations of the network of neuronal voltages driven by Poisson spikes (see figure 3.9). The difference between the theoretically predicted firing rate and the numerically measured firing rate arises from the approximation of the KFE. The error between the two becomes negligible as the fluctuations become smaller, as seen in the case of $f=0.002$ in figure 3.9. For larger values of $f$, the diffusion approximation is no longer valid. In addition, when $f=0.01$, the numerically measured firing rate begins to deviate from the theoretical firing rate as $f \nu$ decreases. This deviation from the theory can be attributed to the fact that the network becomes less synchronizable as $f \nu$ decreases for fixed $f$ in the subthreshold regime.

\section{Asynchronous regime}

In this section, we discuss the properties of the steady, asynchronous solutions of the I\&F model (2.1), (2.3), both because they are interesting in their own right and to contrast them with the synchronous solutions discussed in the previous section. In particular, as will be seen below, for sufficiently small external drive fluctuations the gain curves corresponding to steady asynchronous solutions exhibit bistability and hysteresis, which we have not been able to find for the gain curves of the synchronized solutions. Moreover, in the regime of sufficiently strong average external current, $f \nu$, the gain curves asymptotically depend on the mean of the drive alone in contrast to the gain curves for the synchronized solutions, which are always sensitive to the fluctuations of the drive. Analogous results for the conductance-based I\&F model are described in [49].

We have observed that our idealized I\&F model, (2.1) and (2.3), operates asynchronously only for small network coupling strengths and relatively large external drive fluctuations. When the external drive fluctuations become small, i.e., $f \ll 1$, numerical simulations indicate that the basin of attraction of the asynchronous so- 


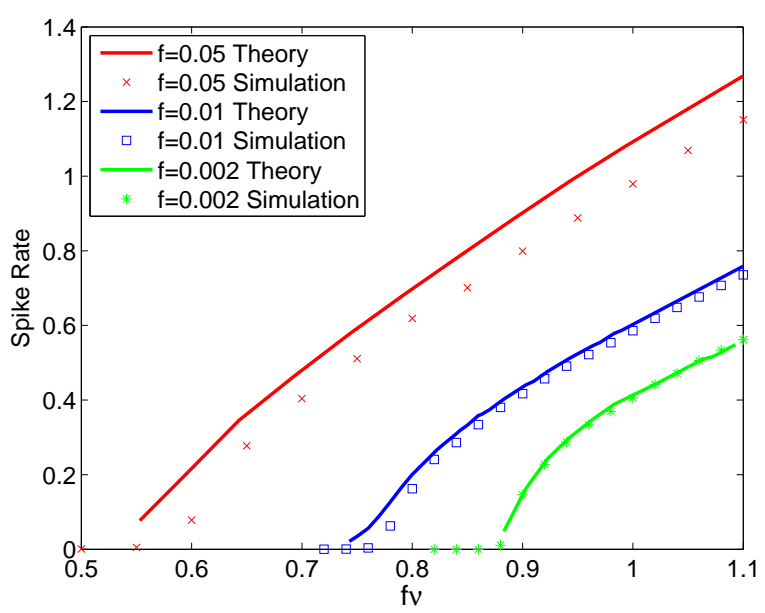

FIG. 3.9. For the network of $N=100$ neurons with network coupling strength $S=2.0$, the firing rate determined by the procedure of section 3.2 is in good agreement with measurements obtained from numerical simulation of system (2.1) in the small-fluctuation regime for which the diffusion approximation is valid.

lution also becomes small. In particular, any chance of observing this solution and its bistability appears to be diminished by the model's tendency to synchronize. We therefore incorporate some more realistic physiological effects into the model that suppress the synchrony and enlarge the basin of attraction of the asynchronous solution. Instead of increasing the size of $f$ and decreasing the network size $N$, we will see that synaptic transmission delays sustain the asynchronous dynamics in this work. We postulate the relevant KFE, study its steady dynamics, and compute gain curves in order to determine the voltage PDF and the firing rate of a typical neuron in the network.

We should point out two immediate differences between the synchronous and asynchronous regimes. First, the network activity in the asynchronous regime can be described by a steady-state voltage PDF, while, as we have seen in the synchronous regime, the voltage PDF depends on time in a nearly periodic fashion. Also, the main statistical quantity we will directly compute for the asynchronous regime is the average firing rate of the network, as opposed to the average time between synchronous total firing events.

4.1. Network with transmission delays. The simplest description of the asynchronous regime in the network (2.1), (2.3) can be obtained if the train of current spikes received by any neuron in the network can be assumed to satisfy Poisson statistics in time. Under this assumption, we can find a KFE that describes the voltage PDF in the asynchronous regime. We have been modeling the spike times of the external drive as a Poisson train from the outset, and the internal spikes can be approximated by such a process if they come from a large number of independent weak sources. That is, each neuron in the network must fire independently, and a large number of infrequently firing neurons must contribute to the current of every neuron in the network $[25,53],[44$, Sec. 5.9]. Note that this assumption is clearly violated in the synchronous regime. However, another intrinsic obstacle to independent firing of 
neurons in the network (2.1), (2.3), is that any neuron can only spike precisely at the moment when an external spike or a spike from another network neuron arrives. This may well be the reason underlying the difficulty, if not impossibility, of finding a regime in which the spike train of the total output generated by the entire network (2.1), (2.3) obeys Poissonian statistics. In particular, we observe that numerical simulations of the network (2.1), (2.3) lock onto synchronous or near-synchronous operation much more readily than asychronous operation for small external drive and very small network coupling.

Several heretofore ignored physiological phenomena can be included in the model (2.1), (2.3) that remove the strong tendency of the network to synchronize and render the asynchronous state more easily observable. One such effect is sparsity of the neuron-to-neuron connections in the network, so that the connectivity of the network ceases to be all-to-all $[5,13,93,94]$. Another is significant synaptic transmission failure (2.3), i.e., random failure of a neuron's synaptic response to a presynaptic spike. To model this failure, the current driving the $j$ th neuron in equation $(2.3)$ is replaced with a current of the form

$$
I_{j}(t)=f \sum_{l} \delta\left(t-s_{j l}\right)+\frac{S}{N p} \sum_{i \neq j} \sum_{k} p_{j i k} \delta\left(t-\tau_{i k}\right),
$$

where $p_{j i k}$ is a Bernoulli-distributed stochastic variable modeling the synaptic release probability, i.e., the probability of current activation upon receiving a spike $[3,41$, $62,69,74,96]$. In particular, $p_{j i k}=1$ with probability $p$ and 0 with probability $1-p$. However in our simulations these two effects, synaptic sparsity and synaptic failure, are not very effective in preventing the system from locking onto synchronous dynamics.

We find that inserting transmission delays into the currents driving the neurons in the network can efficiently sustain the network dynamics in an asynchronous regime. Rather than for one neuron to instantaneously pass information to another, the passing of information takes a random amount of time due to the axonal velocity and axonal length distribution $[12,29,56]$. Random transmission delays in the $j$ th neuron's synaptic current are modeled by replacing the current (2.3) with

$$
I_{j}(t)=f \sum_{l} \delta\left(t-s_{j l}\right)+\frac{S}{N} \sum_{i \neq j} \sum_{k} \delta\left(t-\tau_{i k}-T_{j i k}\right),
$$

where $T_{j i k}$ is a non-negative stochastic variable taken from some appropriate distribution. In this work, we model each delay time $T_{j i k}$ to be exponentially distributed with common mean. Note that, as these delay times are all assumed to be independent, it is possible for the spikes to be transmitted in a different order than they were originally generated.

Including random transmission delays into the model does however present one theoretical difficulty to the KFE approach. Namely, the input from other neurons to a neuron in the network would be a convolution of firing history in time. However, in the steady state where the firing rate, $m$, does not depend on time, each neuron is effectively driven by a mean firing rate, $m$, regardless of the delay dynamics. Moreover, if the average delay time is significantly shorter than the time scale on which the network firing rate, $m(t)$, varies, we can also assume to a good degree of approximation that each neuron is driven by a mean firing rate, $m(t)$. In what is to follow, we will assume that the network satisfies one of these conditions, usually the steady-state one. 
In the forthcoming sections we first derive the KFE, in the diffusion approximation, describing the steady voltage PDF in the asynchronous state of the network (2.1), (4.1) with random transmission delays. We then present the exact, explicit formulas for the voltage PDF and gain curves in the limit of vanishing fluctuations, the exact voltage PDF solution of the full KFE in the diffusion approximation, and an exact implicit equation for the gain curves, which we solve numerically. Finally, in two asymptotic regimes that cover almost the entire gain curve, we find approximate expressions for the voltage PDF and the gain curve shape in terms of elementary functions, which are valid asymptotically for small drive fluctuations.

4.2. Steady Kolmogorov forward equation. We are interested in obtaining the voltage PDF of a typical neuron and studying the firing rate of the asynchronous current-based network, both of which can be derived from the solution to the KFE. In a large network of coupled neurons which fire asynchronously, the average network firing rate appears as a self-consistent part of the driving term in the KFE. Thus by solving the steady-state KFE with the appropriate boundary and normalization conditions as discussed below, the steady-state average network firing rate of the network as well as the stationary distribution of a typical neuronal voltage are obtained. We first postulate the KFE that takes into account finite voltage jumps due to the external drive and network spikes. We then approximate these jumps by drift and diffusion terms in the limit of small fluctuations, and finally derive a simplified KFE satisfied by the steady voltage PDF.

When the network, (2.1), (4.1), fires asynchronously, a KFE can be derived [57,58] for the PDF of the voltage of a typical neuron in the network, $p_{v}(x, t)$. This equation is

$$
\begin{aligned}
\frac{\partial}{\partial t} p_{v}(x, t)= & \frac{\partial}{\partial x}\left[g_{L}\left(x-V_{R}\right) p_{v}(x, t)\right]+\nu\left[p_{v}(x-f, t)-p_{v}(x, t)\right] \\
& +N m(t)\left[p_{v}\left(x-\frac{S}{N}, t\right)-p_{v}(x, t)\right]+m(t) \delta\left(x-V_{R}\right) .
\end{aligned}
$$

Here, $N m(t)$ is the instantaneous firing rate of the entire network, so that $m(t)$ is the population-averaged firing rate per neuron. The delta-function term is the probability source due to the resetting of the neuronal voltages at $V_{R}$ after they have crossed the firing threshold, $V_{T}$.

Equation (4.2) can be written in the conservation form

$$
\frac{\partial}{\partial t} p_{v}(x, t)+\frac{\partial}{\partial x} J\left[p_{v}\right](x, t)=m(t) \delta\left(x-V_{R}\right)
$$

using the probability flux

$$
J\left[p_{v}\right](x, t)=J_{d}\left[p_{v}\right](x, t)+J_{u}\left[p_{v}\right](x, t) .
$$

Here,

$$
J_{d}\left[p_{v}\right](x, t)=-g_{L}\left(x-V_{R}\right) p_{v}(x, t)
$$

is the downwards flux of the neuronal voltages due to the relaxation dynamics in equation (2.1), and

$$
J_{u}\left[p_{v}\right](x, t)=\nu \int_{x-f}^{x} p_{v}(s, t) d s+N m(t) \int_{x-S / N}^{x} p_{v}(s, t) d s
$$


is the flux due to upwards jumps induced by the delta-function currents arriving from both the external input and the spikes of the network neurons.

We now consider the boundary conditions for equation (4.2). The I\&F mechanism resets the voltages of all neurons that have crossed the firing threshold, $V_{T}$, immediately to the value $V_{R}$. Therefore, there are no neuronal voltages above $V_{T}$ to relax down past the boundary $V_{T}$, and thus no downward flux at $V_{T}$. From equation (4.5), we thus find

$$
J_{d}\left[p_{v}\right]\left(V_{T}, t\right)=-g_{L}\left(V_{T}-V_{R}\right) p_{v}\left(V_{T}, t\right)=0,
$$

and hence,

$$
p_{v}\left(V_{T}, t\right)=0
$$

which is an absorbing boundary condition for the KFE (4.2) at $V_{T}$. The second boundary condition for (4.2) is derived by noting that the voltage probability flux over the threshold, $V_{T}$, must equal the firing rate,

$$
J\left[p_{v}\right]\left(V_{T}, t\right)=J_{u}\left[p_{v}\right]\left(V_{T}, t\right)=m(t) .
$$

As mentioned above, the reinjection of these voltages into the equation at the reset value, $V_{R}$, is taken care of by the delta-function term in equation (4.2).

In addition to the boundary conditions (4.8) and (4.9), the voltage PDF, $p_{v}(x, t)$, must also be non-negative in the $x$-interval $\left[V_{R}, V_{T}\right]$, and satisfy the normalization condition

$$
\int_{V_{R}}^{V_{T}} p_{v}(x, t) d x=1 .
$$

It is through this normalization condition, computed over the voltage interval $\left[V_{R}, V_{T}\right]$, that the firing rate $m(t)$ is self-consistently determined.

Normalization (4.10) stands in contrast with the voltage PDF which solves the KFE obtained in sections 3.2.2. In this latter PDF, the neurons which traversed the firing threshold were not returned to the interval $\left[V_{R}, V_{T}\right]$, thus the area under the curve $p_{v}(x, t)$ in the voltage interval, $\left[V_{R}, V_{T}\right]$ decreased with time and was only normalized to unity initially at $t=0$. For the purpose of the analysis in section 3.2 .2 , the reinjection of neuronal voltages at reset $V_{R}$ is irrelevant, and the leaking of the voltage PDF determines, through (3.42), the probability distribution for the first neuron firing time through the rate of decrease of the integral in equation (3.39). This was one of the crucial ingredients in our calculation of the period between two total firing events. The voltage PDF, $p_{v}(x, t)$, derived in this section describes the actual distribution of the neuronal voltages complete with the reseting of voltage to $V_{R}$ upon crossing the threshold, $V_{T}$, thus it remains normalized to unity for all time.

In the present work, rather than try to solve the difference equation (4.2) directly, we will follow the procedure in section 3.2.2, when the voltage jumps $f$ and $S / N$ are small (small-fluctuation regime), and Taylor expand the difference terms in equation (4.2) to second order. The delta function is treated in the new boundary condition, as explained below. This leads to the approximate KFE

$$
\begin{aligned}
\frac{\partial}{\partial t} p_{v}(x, t)= & \frac{\partial}{\partial x}\left[\left(g_{L}\left(x-V_{R}\right)-f \nu-S m(t)\right) p_{v}(x, t)\right] \\
& +\frac{1}{2}\left(f^{2} \nu+\frac{S^{2} m(t)}{N}\right) \frac{\partial^{2}}{\partial x^{2}} p_{v}(x, t),
\end{aligned}
$$


which we rewrite in the form

$$
\tau \frac{\partial}{\partial t} p_{v}(x, t)=\frac{\partial}{\partial x}\left[(x-\mu(t)) p_{v}(x, t)+\sigma^{2}(t) \frac{\partial}{\partial x} p_{v}(x, t)\right]
$$

with the new parameters

$$
\tau=1 / g_{L}, \quad \mu(t)=V_{R}+\frac{f \nu+S m(t)}{g_{L}}, \quad \sigma^{2}(t)=\frac{1}{2 g_{L}}\left(f^{2} \nu+\frac{S^{2} m(t)}{N}\right) .
$$

Under the assumption that the average external current $f \nu$, the network coupling coefficient $S$, and the population-averaged firing rate $m(t)$ all remain $O(1)$, the above assumption of small jumps implies that $\sigma^{2}(t) \ll 1$ and $\mu(t)$ is of $O(1)$. In other words, we consider equation (4.11), which is of Fokker-Planck type, in the small-fluctuation regime. We generally also wish to consider the mean input current $S m$ arriving from other neurons to be $O(1)$, otherwise the network is operating in an essentially trivial feedforward mode. This means that the diffusion approximation (4.11) is appropriate for networks with $f$ small and $N$ large, with $f \nu, m$, and $S$ order unity. In particular, a necessary asymptotic condition is that $f \ll\left(V_{T}-V_{R}\right)$ and $S / N \ll\left(V_{T}-V_{R}\right)$, meaning that a large number of incoming spikes from either the external input or other neurons is needed to drive a given neuron from the reset voltage up to threshold.

We remark that a KFE similar to equation (4.11) can also be derived for more general I\&F type systems, for example, those with a finite conductance or current time course, via kinetic theory $[1,5,6,14,21-24,33,36,42,46,57,58,60,61,68,70,72,98]$. For conductance-based networks, the equation corresponding to (4.11) is derived via a closure that eliminates the conductance variables and a subsequent assumption of infinitely short conductance time-scales $[21,22,70]$.

The conservation form of equation (4.11) is

$$
\frac{\partial}{\partial t} p_{v}(x, t)+\frac{\partial}{\partial x} J\left[p_{v}\right](x, t)=0
$$

with the approximate probability flux

$$
J\left[p_{v}\right](x, t)=-\frac{1}{\tau}\left[(x-\mu(t)) p_{v}(x, t)+\sigma^{2}(t) \frac{\partial}{\partial x} p_{v}(x, t)\right]
$$

being the counterpart of the flux (4.4).

The boundary conditions for equation (4.11) are different from those for equation (4.2). Consider the lower boundary where, previously, a delta function took care of reset neurons. By integrating across the boundary, this term is replaced by the flux across the boundary, namely $m(t)$, the rate at which neurons are reset. At the upper boundary, the boundary condition on the flux remains the same. This gives the boundary condition

$$
J\left[p_{v}\right]\left(V_{T}, t\right)=J\left[p_{v}\right]\left(V_{R}, t\right)=m(t),
$$

which states that the rate at which neurons leave the upper boundary (fire) is the rate at which they appear at the lower boundary (reset). The absorbing boundary condition (4.8) is kept for the approximate equation (4.11), as is the normalization (4.10).

For constant Poisson rate, $\nu$, of the external forcing spike train, it is natural to seek the simplest solution of the approximate KFE (4.11), which is its steady state. In this 
state the firing rate $m$ as well as $\mu$ and $\sigma$ are no longer time dependent. Since the timederivative on the left-hand-side of (4.11) (or (4.13)) is absent we can integrate (4.13) once and use the flux definition (4.14) and the boundary condition (4.15) to find the equation for the stationary $\mathrm{PDF}$ of the voltage, $p_{s}(x)$, which reads

$$
(x-\mu) p_{s}(x)+\sigma^{2} \frac{\partial}{\partial x} p_{s}(x)=-\tau m \text {. }
$$

The solution of equation (4.16) must also satisfy the absorbing boundary condition (4.8) at the firing threshold $V_{T}$ and the normalization (4.10).

In what is to follow, we will address the solutions of equation (4.16), in particular, the gain curves depicting the dependence of the steady-state firing rate $m$ on the external input strength $f \nu$, beginning with the zero-fluctuation limit in section 4.3 , exact solution of equation (4.16) in section 4.4, and two asymptotic approximations in section 4.5 .

4.3. Zero-fluctuation limit.

Before addressing the exact solution of equation (4.16), we consider the limit in which the fluctuations in the driving current go to zero, such that the overall drive strength $f \nu+S m(t)$ remains order unity. In this limit, the coefficient $\sigma^{2}$ defined in equation (4.12), which measures the size of the fluctuation term in equations (4.11) and (4.16), vanishes. The size of the fluctuations is governed by the size of the terms $f^{2} \nu$ and $S^{2} m(t) / N$ in $\sigma^{2}$, so the relevant limit is $f \rightarrow 0, \nu \rightarrow \infty$, and $N \rightarrow \infty$, while $f \nu+S m(t)$ remains order unity. The average network firing rate is equivalent to the firing rate of the same network driven by an effective constant external current of strength $f \nu+S m(t)$. As we will see, the gain curve in this limit consists of two segments: a vanishing segment in the interval $0<f \nu<g_{L}\left(V_{T}-V_{R}\right)$, and a mean-driven segment emanating from the point $f \nu=g_{L}\left(V_{T}-V_{R}\right), m=0$. (The term "mean-driven" will be explained below.)

Equation (4.16) in the zero-fluctuation limit loses its derivative term and becomes

$$
p_{s}(x)=\frac{\tau m}{\mu-x}
$$

The boundary condition (4.8) must naturally be dropped. If the firing rate $m$ does not vanish, this solution is non-singular for $x \in\left[V_{R}, V_{T}\right]$ only when $\mu>V_{T}$. According to equation (4.12), this occurs when

$$
f \nu+S m>g_{L}\left(V_{T}-V_{R}\right)
$$

i.e., when the combined external and network spikes drive the voltage of a single network neuron across threshold on average. The normalization condition (4.10) applied to the PDF (4.17) yields the equation

$$
1=m \tau \ln \frac{\mu-V_{R}}{\mu-V_{T}}=m \tau \ln \frac{f \nu+S m}{f \nu+S m-g_{L}\left(V_{T}-V_{R}\right)},
$$

which is an implicit equation for the firing rate $m$. Since only the mean of the external drive, $f \nu$, enters this equation, we refer to the regime described by equation (4.19), in which $m>0$, as the mean-driven regime.

Defining the parameter

$$
\Delta=g_{L}\left(V_{T}-V_{R}\right),
$$



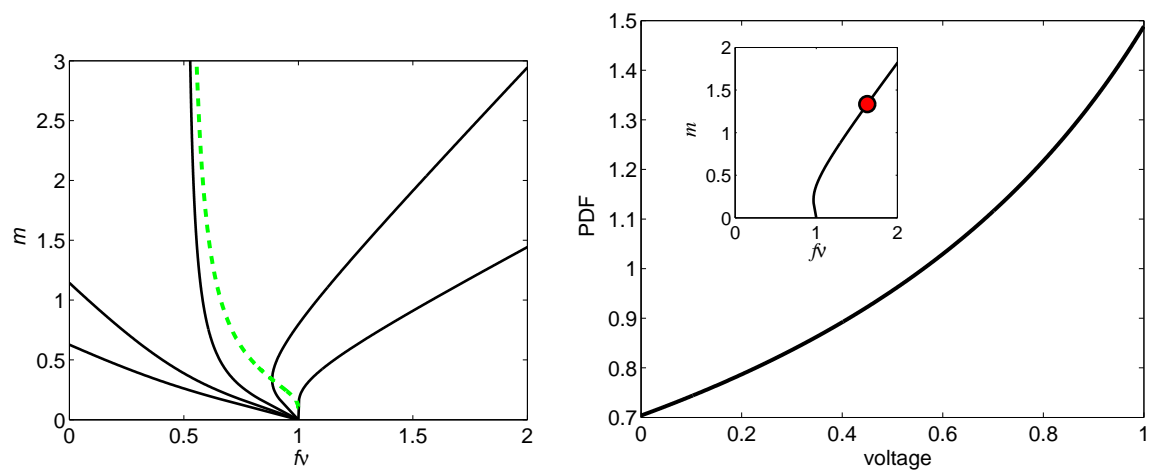

FIG. 4.1. (Left) Gain curves computed via formula (4.21) for left to right: $S=2.0,1.5,1.0,0.5$, and $S=0$. The light grey (green online) dashed curve indicates the location of the turning point. (Right) A representative graph of voltage PDF computed from (4.17) with its location along the gain curve with $S=0.2$ in the inset.

which is the threshold for the external drive strength, we find that equation (4.19) can be solved exactly in the form

$$
f \nu=\frac{\Delta}{1-\exp (-1 / m \tau)}-S m
$$

A number of representative gain curves, computed from equation (4.21), for different values of the network coupling constant $S$ are displayed in figure 4.1, which also shows a representative graph of the voltage PDF as computed from formula (4.17). The gain curves all begin at the point $f \nu=\Delta, m=0$, and go backwards in $f \nu$ along the unstable stretch of the bistable region. For each value of the network coupling constant $S$, the derivative of the corresponding gain curve at this point equals $-1 / S$. For large values of the firing rate $m$, each gain curve approaches its straight-line asymptote given by the equation

$$
m=\frac{f \nu-\Delta / 2}{\tau \Delta-S} .
$$

This asymptote has a positive slope if $S<\tau \Delta$ and negative slope if $S>\tau \Delta$. If $S=\tau \Delta$, the asymptote is the vertical line $f \nu=\Delta / 2$. This shows that, for $S<\tau \Delta$, the gain curve exhibits bistability and has a hysteretic regime for $f \nu<\Delta$, as its slope turns from negative to positive in a saddle-node bifurcation. For $S>\tau \Delta$, the gain curve has a negative slope along its entire length, and intercepts the line of vanishing firing rate. The curve corresponding to an uncoupled neuron, $S=0$, has no bistability region. With its derivative being infinite at $f \nu=\Delta, m=0$, this gain curve increases monotonically with a monotonically decreasing slope, eventually approaching the straight line

$$
m=\frac{f \nu-\Delta / 2}{\tau \Delta} .
$$

Bistability in a similar model was found in [50]. We should also remark that, in network simulations, downward sloping segments (i.e. with negative derivatives) of the gain curves are unstable and therefore cannot be directly observed. 


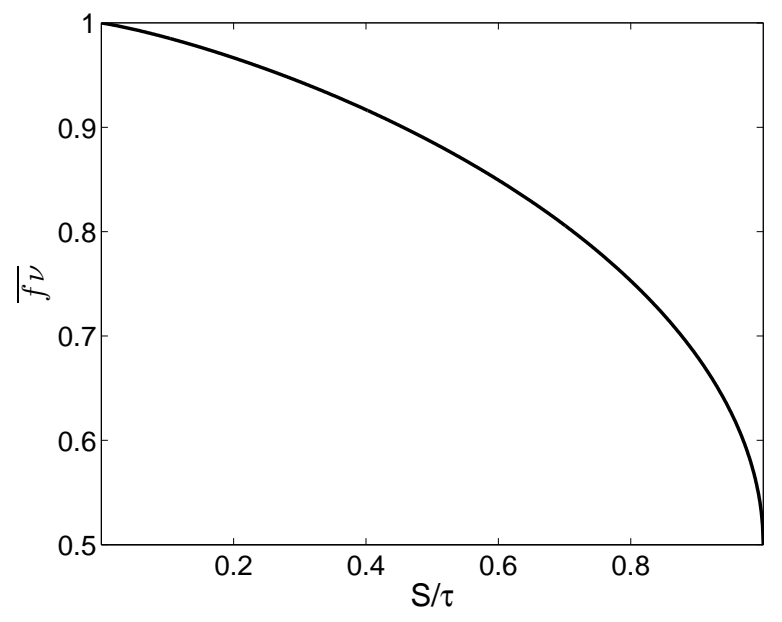

FIG. 4.2. The $f \nu$-coordinate, $\overline{f \nu}$, of the gain-curve turning point as a function of the parameter ratio $S / \tau$ where $\tau=1 / g_{L}$.

The external drive strength corresponding to the turning point on the gain curve, i.e., the saddle node bifurcation, can be determined by finding the minimum of the expression in equation (4.21). From this equation, it is clear that the $f \nu$-coordinate, $\overline{f \nu}$, of the turning point is only a function of the parameter ratio $S / \tau$. In figure 4.2 , we display the functional dependence of $\overline{f \nu}$ on $S / \tau$. The bifurcation curve begins at the point $S / \tau=0, \overline{f \nu}=\Delta$, slopes downwards, and ends at the point $f \nu=\Delta / 2$, $S / \tau=\Delta$.

When the firing rate, $m$, vanishes, equation (4.17) cannot be used to determine the voltage PDF. Instead, we study this situation directly using the network (2.1), (2.3). Replacing the Poisson external drive train in the current (2.3) by the mean current $f \nu$ and taking into account that network spikes do not occur, we find that in this case all the neuronal voltages in the network are driven towards the equilibrium voltage $V_{R}+f \nu / g_{L}$, where, clearly, we must have $f \nu / g_{L}<V_{T}-V_{R}$, or else the assumption $m=0$ would be violated. As a consequence, in this subthreshold driving regime, the steady voltage PDF becomes

$$
p_{s}(x)=\delta\left(x-\frac{f \nu}{g_{L}}\right) .
$$

Thus, all gain curves share an additional segment, which is the interval $0<f \nu<\Delta$ with $m=0$. As will be seen in sections 4.4 and 4.5.1, this segment is the limiting case of the fluctuations-driven regime along the gain curve: the firing rate $m$ vanishes along this segment in the present case because the fluctuations are absent.

The assumption of vanishing fluctuations made in this section gave us an approximate explicit description of the gain curves and voltage PDFs. In the next section, we will find the exact solution of equation (4.16) for the voltage PDF, and from it compute a more accurate set of gain curves.

4.4. Exact voltage PDF and gain curves. The above zero-fluctuation limit does not describe why the network fires when $f \nu+S m<\Delta$, i.e., in the fluctuations- 
driven regime. To accurately describe the fluctuations-driven regime dynamics, we address the exact solution to the steady-state KFE (4.16) and compute the corresponding gain curves. Using equation (4.16) and the absorbing boundary condition (4.8), we find the stationary voltage $\mathrm{PDF}, p_{s}(x)$, to be

$$
p_{s}(x)=\frac{\sqrt{2} m \tau}{\sigma} \exp \left(-\frac{(x-\mu)^{2}}{2 \sigma^{2}}\right) \int_{(x-\mu) / \sqrt{2} \sigma}^{\left(V_{T}-\mu\right) / \sqrt{2} \sigma} \exp \left(s^{2}\right) d s,
$$

which agrees with the result of [14]. This PDF can be rewritten as

$$
p_{s}(x)=\frac{\sqrt{2} m \tau}{\sigma}\left[\exp \left(\frac{\left(V_{T}-\mu\right)^{2}}{2 \sigma^{2}}\right) D\left(\frac{V_{T}-\mu}{\sqrt{2} \sigma}\right) \exp \left(-\frac{(x-\mu)^{2}}{2 \sigma^{2}}\right)-D\left(\frac{x-\mu}{\sqrt{2} \sigma}\right)\right],
$$

where $D(\cdot)$ denotes the Dawson integral

$$
D(z)=e^{-z^{2}} \int_{0}^{z} e^{y^{2}} d y
$$

and $\mu$ is defined in (4.12).

To find an equation for the corresponding gain curve, we use the normalization condition (4.10) and integrate equation (4.24). Defining the parameter

$$
a=f \nu+S m,
$$

which denotes the average current arriving at a network neuron, and noticing that equation (4.12) is equivalent to $\mu=V_{R}+a / g_{L}=V_{R}+\tau a$, we thus obtain the equation

$$
\begin{aligned}
\int_{V_{R}}^{V_{T}} p_{s}(x) d x=m \tau & \left\{\sqrt { \pi } \operatorname { e x p } ( \frac { ( \Delta - a ) ^ { 2 } } { 2 \sigma ^ { 2 } g _ { L } ^ { 2 } } ) D ( \frac { \Delta - a } { \sqrt { 2 } \sigma g _ { L } } ) \left[\operatorname{erf}\left(\frac{\Delta-a}{\sqrt{2} \sigma g_{L}}\right)\right.\right. \\
& \left.\left.+\operatorname{erf}\left(\frac{a}{\sqrt{2} \sigma g_{L}}\right)\right]-2 \int_{-a / \sqrt{2} \sigma g_{L}}^{(\Delta-a) / \sqrt{2} \sigma g_{L}} D(s) d s\right\} \\
=m \tau & \left\{\sqrt { \pi } \operatorname { e x p } ( \frac { ( \Delta - a ) ^ { 2 } } { 2 \sigma ^ { 2 } g _ { L } ^ { 2 } } ) D ( \frac { \Delta - a } { \sqrt { 2 } \sigma g _ { L } } ) \left[\operatorname{erf}\left(\frac{\Delta-a}{\sqrt{2} \sigma g_{L}}\right)\right.\right. \\
& \left.+\operatorname{erf}\left(\frac{a}{\sqrt{2} \sigma g_{L}}\right)\right]-\frac{(\Delta-a)^{2}}{2 \sigma^{2} g_{L}^{2}}{ }_{2} F_{2}\left([1,1],\left[\frac{3}{2}, 2\right],-\frac{(\Delta-a)^{2}}{2 \sigma^{2} g_{L}^{2}}\right) \\
& \left.+\frac{a^{2}}{2 \sigma^{2} g_{L}^{2}}{ }_{2} F_{2}\left([1,1],\left[\frac{3}{2}, 2\right],-\frac{a^{2}}{2 \sigma^{2} g_{L}^{2}}\right)\right\}=1 .
\end{aligned}
$$

Here, $\operatorname{erf}(\cdot)$ denotes the error function, defined in equation (3.21), and we have used the formula [97]

$$
\int D(z) d z=\frac{z^{2}}{2}{ }_{2} F_{2}\left([1,1],\left[\frac{3}{2}, 2\right],-z^{2}\right),
$$

where ${ }_{k} F_{l}$ is the generalized hypergeometric function

$$
{ }_{k} F_{l}\left(\left[\alpha_{1}, \ldots, \alpha_{k}\right],\left[\beta_{1}, \ldots, \beta_{l}\right], z\right)=\sum_{n=0}^{\infty} \frac{\left(\alpha_{1}\right)_{n} \cdots\left(\alpha_{k}\right)_{n}}{\left(\beta_{1}\right)_{n} \cdots\left(\beta_{l}\right)_{n}} \frac{z^{n}}{n !},
$$




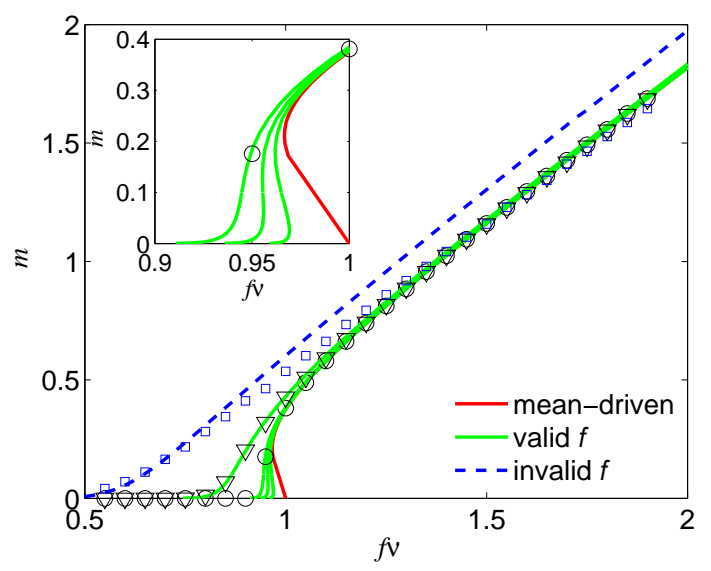

FIG. 4.3. Gain curves for different strengths of fluctuations. Left to right: $f=0.1, N=10$; $f=0.01, N=100 ; f=0.001, N=1000 ; f=0.0005, N=2000 ; f=0.0002, N=5000 ;$ no fluctuations. Solid and dashed curves: theory; squares, triangles and circles: measurements from numerical simulations. For all curves $S=0.2$. For the invalid value of $f$, the diffusion approximation no longer holds. The insert zooms in around $f \nu=0.95$.

with $(\gamma)_{j}$ being the Pochhammer symbol defined in equation (3.71). Upon expressing the parameters $a$ and $\sigma$ in terms of the driving strength $f \nu$ and firing rate $m$ from equations (4.12), (4.26), and (4.20), formula (4.27) gives an implicit equation connecting $m$ and $f \nu$.

To compute the gain curves in practice, it is more convenient to numerically compute the root of equation (4.10) after first numerically integrating equation (4.24), with the parameters $\mu$ and $\sigma$ again expressed in terms of the average external current $f \nu$ and firing rate $m$ from equation (4.12). The resulting gain curves are depicted in figure 4.3 for different values of the external spike strength, $f$, and correspondingly large network sizes, $N$. The gain curves corresponding to small values of $f$ and large values of $N$ are close to their zero-fluctuation limit counterparts, except that they are smooth near the point $f \nu=\Delta=g_{L}\left(V_{T}-V_{R}\right), m=0$. Thus, they still behave in a hysteretic, bistable fashion. The gain curves for larger values of $f$ and smaller $N$ lose their bistable stretches.

Figure 4.3 also shows comparison of the gain curves predicted theoretically with those obtained from simulations for different strengths of fluctuations. We see that, indeed, the agreement is excellent for small values of the external spike strength, $f$, and large network sizes, $N$, i.e., small fluctuations. When the fluctuations increase, there is a discrepancy, which is the most pronounced in the location of the gain curve at high values of the external drive: the true gain curve obtained from the simulations follows closely the zero-fluctuation limiting curve for large $f \nu$. This discrepancy is the result of the failure of the diffusion approximation when the voltage jumps induced by incoming currents, i.e., the individual voltage fluctuations, are large.

Figure 4.4 shows a comparison of the theoretically-predicted and simulated gain curves for different values of the network coupling strength, $S$, in the small-fluctuations regime. The agreement is excellent, including the existence and endpoints of the bistability intervals. Note that the two stable simulated gain curve branches have been obtained by ramping the external drive strength, $f \nu$, up and down. The downwards 


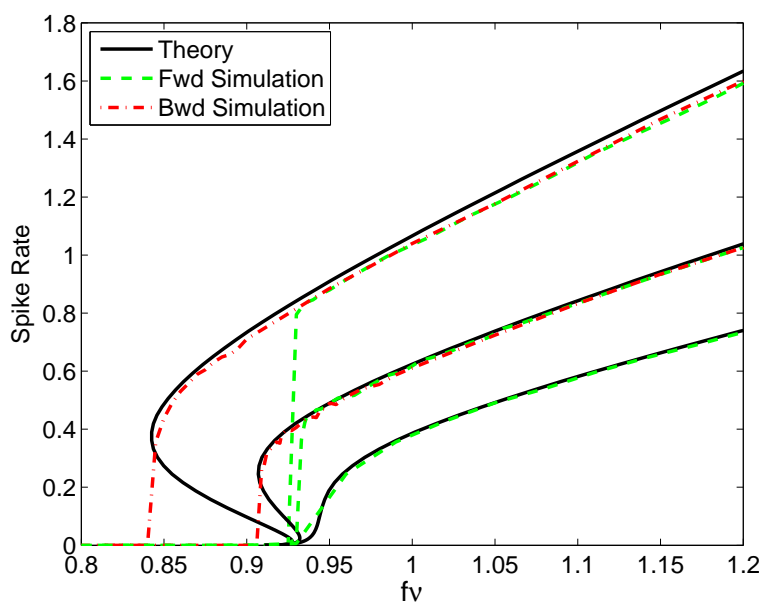

FIG. 4.4. Comparison between gain curves obtained theoretically (solid curves) and from numerical simulations (dashed and dash-dotted curves) from top to bottom: $S=0.6,0.4,0.2$. The dashed curves were obtained by increasing the value of external drive $f \nu$ in subsequent simulations, the dash-dotted curves by decreasing it. When one branch loses stability, the simulations lock onto the solution on the remaining stable branch. The rest of the parameters are $N=100$ and $f=0.001$.

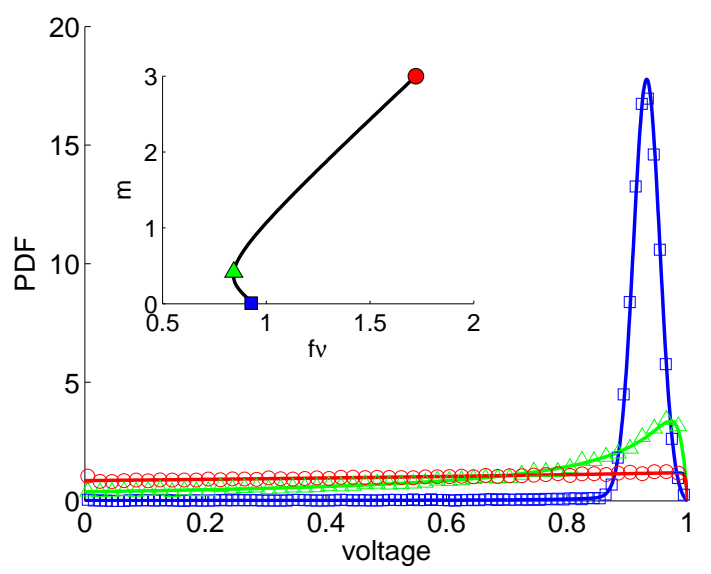

FIG. 4.5. Three voltage PDF curves along a gain curve. Solid curves were obtained from formula (4.24), symbols from numerical simulations. The black curve (blue online) and squares are in the fluctuations-driven regime, the other two are in the mean-driven regime. The inset plots the corresponding locations on the gain curve. The parameter values are $f=0.001, S=0.6$, and $N=100$.

sloping middle branch of the gain curve is always unstable and cannot be obtained directly via numerical simulations.

Finally, figure 4.5 displays three voltage PDFs at various locations along the gain curve in the limit of small fluctuations. Note that, in the fluctuations-driven regime, the PDF strongly resembles a Gaussian, while deep into the mean-driven regime, the 
PDF resembles the solution (4.17) displayed in figure 4.1, except for a thin boundary layer near the firing threshold, $V_{T}$.

The exact solutions obtained in this section are really only necessary to accurately describe the transition between the fluctuations-driven regime and the unstable branch of the mean-driven regime near the corner point, $f \nu=\Delta, m=0$, of the zerofluctuation gain curve. Along the stable branches of both the fluctuations- and meandriven regimes, the expressions for both the voltage PDFs and gain curves will simplify considerably, as we will see next.

4.5. Asymptotic regimes. The results of section 4.4 are valid for all values of the external drive $f \nu$, in both the fluctuations- and the mean-driven regimes, as long as the network operates in the small-fluctuations regime, which is equivalent to $\sigma^{2} / a \ll 1$. In this section we consider approximations of the exact voltage PDF and gain curves by elementary functions in both the fluctuations-driven regime, $0<a<\Delta$, and the mean-driven regime, $a>\Delta$.

In both regimes, we use the following asymptotic expressions:

$$
D(z) \sim \frac{1}{2 z}, \quad|z| \gg 1
$$

and

$$
\operatorname{erf}(z) \sim \operatorname{sign}(z)-\frac{1}{\sqrt{\pi} z} e^{-z^{2}}, \quad|z| \gg 1
$$

and thus avoid needing to evaluate either of these two functions directly.

We remark that neither of the two asymptotic approximations of the gain curve we compute below are valid near the turning point between the lower stable branch and the unstable branch in the vicinity of the point $f \nu=\Delta, m=0$. The exact gain curve must be used to connect these two approximations there.

4.5.1. Fluctuations-driven regime: $0<a<\Delta$. We begin by considering the limit of the voltage PDF, equation (4.24), in this regime, using equation (4.28). Clearly for small values of the fluctuation parameter $\sigma$ the expression in formula (4.24) is the sum of a Gaussian function with the peak amplitude

$$
\sim \frac{m \tau}{\Delta-a} \exp \left(\frac{(\Delta-a)^{2}}{2 \sigma^{2} g_{L}^{2}}\right)
$$

and a function whose size is at most $O(m / \sigma)$, since the Dawson integral (4.25) is known to be a bounded function [2]. After normalizing, the Gaussian thus clearly dominates over the voltage interval $\left[V_{R}, V_{T}\right]$, therefore

$$
p_{s}(x) \sim \frac{m \tau}{\Delta-a} \exp \left(\frac{(\Delta-a)^{2}}{2 \sigma^{2} g_{L}^{2}}\right) \exp \left(-\frac{(x-\mu)^{2}}{2 \sigma^{2}}\right) \sim \frac{1}{\sqrt{2 \pi} \sigma} \exp \left(-\frac{(x-\mu)^{2}}{2 \sigma^{2}}\right),
$$

where the prefactor in the expression on the right follows from the normalization condition (4.10).

Using equation (4.30), we can now compute the explicit dependence of the firing rate, $m$, on the external drive strength, $f \nu$, without having to use equation (4.27). In particular, comparing the two prefactors in (4.30) and using the formula $\tau=1 / g_{L}$ immediately implies that the equation for the gain curve is

$$
m \sim \frac{(\Delta-a) g_{L}}{\sqrt{2 \pi} \sigma} \exp \left(-\frac{(\Delta-a)^{2}}{2 \sigma^{2} g_{L}^{2}}\right) .
$$




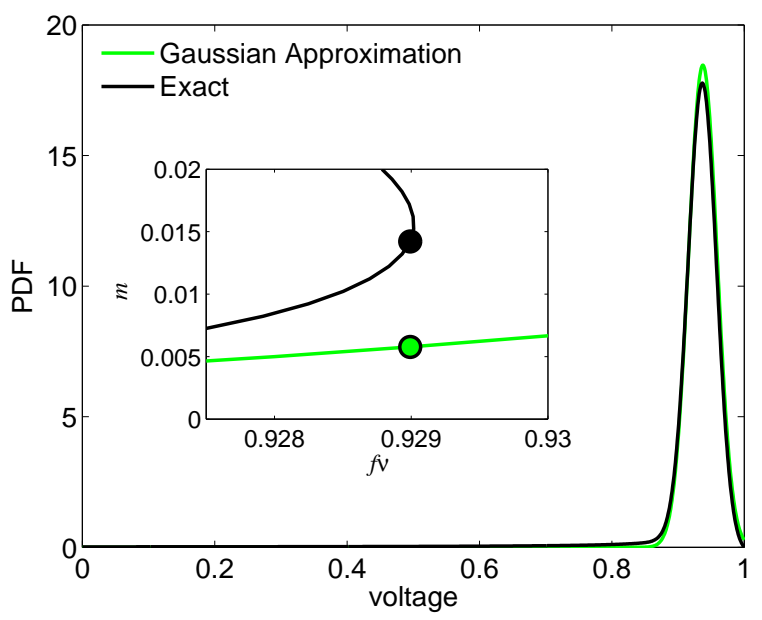

FIG. 4.6. Comparison between the exact voltage PDF in (4.24) (black) and its Gaussian approximation in (4.30) (grey, green online) near the lower turning point of the gain curve. The two gain curves are shown in the inset. The parameter values are $f=0.001, S=0.6, N=1000, g_{L}=1$.

Here, we must use the self-consistent asymptotic values

$$
a \sim f \nu, \quad \text { and } 2 \sigma^{2} \sim f^{2} \nu / g_{L},
$$

which follow from equations (4.26) and (4.12) and the fact that the firing rate, $m$, is exponentially small in $\sigma$, which makes the dependence of $m$ on $f \nu$ in equation (4.31) explicit. This is in agreement with [14].

Figure 4.6 shows the comparison between the exact voltage PDF in (4.24) and its Gaussian approximation in (4.30) near the very end of the external drive interval in which formula (4.31) can serve as an approximation to the exact gain curve and for a small values of $f$. We can see that the agreement between the Gaussian approximation and the exact PDF is still quite good.

In the zero-fluctuation limit, $\sigma \rightarrow 0$ or $f \rightarrow 0$ and $N \rightarrow \infty$, the firing rate in equation (4.31) tends to zero in the interval $0<f \nu<\Delta$, which reproduces the straight-line, $m=0$, segment of the gain curve discussed in section 4.3. Likewise, the voltage PDF (4.30) in this limit approaches the delta-function PDF in (4.22).

4.5.2. Mean driven regime: $a>\Delta$. To compute the small-fluctuations approximation in this regime, we notice that now $\left(V_{T}-\mu\right)^{2}<(x-\mu)^{2}$ throughout the voltage range. Therefore, the first term inside the square brackets in formula (4.24) is exponentially small compared to the second, except at $x=V_{T}$. Using the asymptotic expressions (4.28) and (4.29), we find from equation (4.24) for the voltage PDF, $p_{s}(x)$, the asymptotic expression

$$
p_{s}(x) \sim \frac{\tau m}{\mu-x}\left[1-\exp \left(-\frac{(a-\Delta)\left(V_{T}-x\right)}{\sigma^{2} g_{L}}\right)\right] .
$$

Here, we have also used the asymptotic estimate

$$
\left(V_{T}-\mu\right)^{2}-(x-\mu)^{2} \sim 2\left(V_{T}-V_{R}-a / g_{L}\right)\left(V_{T}-x\right)=2(\Delta-a)\left(V_{T}-x\right) / g_{L},
$$




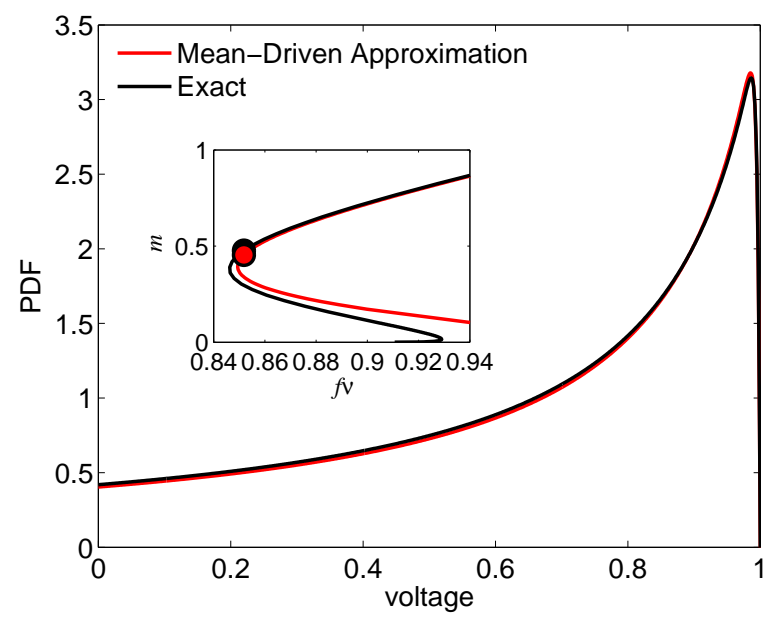

FIG. 4.7. Comparison between the exact voltage PDF in (4.24) (black) and its mean-driven approximation in(4.33) (dark grey, red online) near the lower turning point of the gain curve. The two gain curves are shown in the inset. The parameter values are $f=0.001, S=0.6$ and $N=1000$.

which is valid to $O\left(\left(V_{T}-x\right)^{2}\right)$ when $x \sim V_{T}$.

Expression (4.33) reduces to the zero-fluctuation expression (4.17) away from the firing threshold $x=V_{T}$. The approximate voltage PDF, $p_{s}(x)$ in (4.33), contains an $O\left(\sigma^{2}\right)$ thin boundary layer near the firing threshold $x=V_{T}$. This layer ensures that the boundary condition (4.8) is satisfied.

To find the formula for describing the gain curve, we use the asymptotic expressions (4.28) and (4.29) on the expression in the top two lines of equation (4.27), and integrate the $1 / s$ term obtained from (4.28). Also using (4.26), we find the asymptotic equations for the firing rate in terms of the parameters $a$ and $\sigma^{2}$ in this regime are

$$
m \sim \frac{1}{\tau}\left[\frac{\sigma^{2} g_{L}^{2}}{(a-\Delta)^{2}}+\ln \frac{a}{a-\Delta}\right]^{-1}
$$

and

$$
f \nu \sim a-\frac{S}{\tau}\left[\frac{\sigma^{2} g_{L}^{2}}{(a-\Delta)^{2}}+\ln \frac{a}{a-\Delta}\right]^{-1} .
$$

At the leading order, which is the same order to which equation (4.33) is valid, $a$ becomes a parameter in (4.34), and after eliminating it we recover the explicit solution along the zero-fluctuation limit gain curve, given by equation (4.21).

A comparison between the exact voltage PDF (4.24) and the approximate voltage PDF (4.33) in the mean-driven regime, when the fluctuations are small, is displayed in figure 4.7. We see that even near the lower turning point on the gain curve, the agreement between the two PDFs is excellent.

\section{Absence of chaotic dynamics}

In this section, we show that the current-based I\&F network (2.1), (2.3) coupled with infinitely-fast delta-impulses cannot exhibit chaotic dynamics. We use Lyapunov 
exponents to show this fact, which, as we should point out, is also true in the analogous conductance-based I\&F network [100].

It is well known that Lyapunov exponents measure the average divergence or convergence of nearby orbits in an attractor along the transverse directions in the state space [65, Sec. 3.4.3], [4, Sec. 3.1]. Positive Lyapunov exponents measure the average exponential spreading of nearby trajectories, and negative exponents measure the exponential convergence of trajectories onto the attractor. Generically, the attractor is defined to be chaotic if Lyapunov exponents contain at least one positive exponent, which is also related to the property of sensitive dependence on initial conditions. For non-chaotic attractors, such as periodic or quasi-periodic, Lyapunov exponents contain only vanishing or negative exponents.

For a smooth dynamical system,

$$
\frac{d \boldsymbol{x}(t)}{d t}=\boldsymbol{f}(\boldsymbol{x}(t)), \quad \boldsymbol{x}\left(t_{0}\right)=\boldsymbol{x}_{0},
$$

where $\boldsymbol{f}$ is a continuously differentiable $n$-dimensional vector field, the time evolution of a perturbation (or tangent) vector, $\delta \boldsymbol{x}(t)$, can be represented by the linearization of equation (5.1) as

$$
\delta \dot{\boldsymbol{x}}(t)=\boldsymbol{D} \boldsymbol{f}(\boldsymbol{x}(t)) \cdot \delta \boldsymbol{x} .
$$

Here, $\boldsymbol{D} \boldsymbol{f}$ is the Jacobian matrix of the vector field $\boldsymbol{f}$. The classical largest Lyapunov exponent is defined as

$$
\lambda_{\max }=\lim _{T \rightarrow \infty} \lim _{\epsilon \rightarrow 0} \frac{1}{T} \ln \left(\frac{\|\boldsymbol{x}(T)-\tilde{\boldsymbol{x}}(T)\|}{\epsilon}\right)
$$

with the initial perturbation satisfying $\left\|\boldsymbol{x}\left(t_{0}\right)-\tilde{\boldsymbol{x}}\left(t_{0}\right)\right\|=\epsilon$, and $\tilde{\boldsymbol{x}}(t)$ denoting the perturbed trajectory nearby the reference trajectory $\boldsymbol{x}$. Although this definition is constrained to smooth dynamical systems, we have shown that it can be extended to I\&F network dynamics [101].

In what is to follow, we show that the largest Lyapunov exponent of the network (2.1), and (2.3) is negative and in fact approaches negative infinity for any spike train input. This means that the reference voltage trajectory,

$$
\boldsymbol{v}(t)=\left(v_{1}(t), v_{2}(t), \ldots, v_{N}(t)\right),
$$

and the perturbed voltage trajectory,

$$
\tilde{\boldsymbol{v}}(t)=\left(\tilde{v}_{1}(t), \tilde{v}_{2}(t), \ldots, \tilde{v}_{N}(t)\right),
$$

will converge together after a finite time, provided the magnitude of the initial perturbation size, $\epsilon$, is sufficiently small. Given that the perturbed initial condition, $\tilde{\boldsymbol{v}}\left(t_{0}\right)$, of the network (2.1), (2.3), is a distance $\epsilon$ away the from the reference trajectory, $\boldsymbol{v}\left(t_{0}\right)$, the dynamics of the perturbed trajectory, $\tilde{\boldsymbol{v}}\left(t_{0}\right)$, is described by the equation

$$
\frac{d \tilde{v}_{i}}{d t}=-g_{L}\left(\tilde{v}_{j}-V_{R}\right)+f \sum_{l} \delta\left(t-s_{i l}\right)+\frac{S}{N} \sum_{j \neq i} \sum_{k} \delta\left(t-\tilde{\tau}_{j k}\right)
$$

where $\tilde{\tau}_{j k}$ is the $k^{\text {th }}$ spike of the $j^{\text {th }}$ neuron along $\tilde{\boldsymbol{v}}\left(t_{0}\right)$. The spike times $s_{i l}$ of any (not necessarily Poisson) feedforward input to the $i^{\text {th }}$ neuron are the same as those along the unperturbed reference trajectory, $\boldsymbol{v}(t)$. 
First, we show that the spikes of the reference trajectory, $\boldsymbol{v}(t)$, and the corresponding spikes of the perturbed trajectory, $\tilde{\boldsymbol{v}}(t)$, remain $\mathcal{O}(\epsilon)$-apart for any fixed finite time $T$ as long as $\epsilon$ is sufficiently small, and both the network and feedforward strengths, $S$ and $f$, are finite. Next, we can sort the spike times of both the reference and perturbed trajectories into increasing lists as $\tau_{p_{1} q_{1}} \leq \tau_{p_{2} q_{2}} \leq \cdots \leq \tau_{p_{M} q_{M}}$ and $\tilde{\tau}_{p_{1}, q_{1}} \leq \tilde{\tau}_{p_{2} q_{2}} \leq \cdots \leq \tilde{\tau}_{p_{M} q_{M}}$. Notice that a neuron may fire at exactly the same time as another neuron (say, $p_{k}^{\text {th }}$ neuron and $p_{l}^{\text {th }}$ neuron), thus we have $\tau_{p_{k} q_{k}}=\tau_{p_{l} q_{l}}$. We sort such a case according to the label of the neuron, meaning $p_{k}<p_{l}$ in the list if $\tau_{p_{k} q_{k}}=\tau_{p_{l} q_{l}}$. Therefore the above ordered sequences are unique for each trial.

We now use mathematical induction to prove that the spike times of the reference and perturbed trajectories are exactly the same, namely,

$$
\tilde{\tau}_{p_{m} q_{m}}=\tau_{p_{m} q_{m}} \quad \text { for any } m .
$$

In particular, from the dynamics of equations (2.1), (2.3), and (5.3), we can see that the voltage of both the reference and the perturbed trajectories of any neuron will cross the threshold voltage, $V_{T}$ only when receiving spikes either from the feedforward or network input. Otherwise it will always approach the reset value, $V_{R}$, which is lower than the threshold, $V_{T}$. First, we consider the case $m=1$. Since this is the first firing event, both $\tau_{p_{1} q_{1}}$ and $\tilde{\tau}_{p_{1} q_{1}}$ should be equal to some feedforward input spike time $s_{j k}$. Because the reference and perturbed trajectories receive the same feedforward input, for a sufficiently small difference size, $\epsilon$, we must have $\tilde{\tau}_{p_{1} q_{1}}=\tau_{p_{1} q_{1}}$.

Next, suppose we have $\tilde{\tau}_{p_{m} q_{m}}=\tau_{p_{m} q_{m}}$, and we want to show that $\tilde{\tau}_{p_{m+1} q_{m+1}}=$ $\tau_{p_{m+1} q_{m+1}}$. If the spike of the $p_{m+1}^{\text {th }}$ neuron is caused by the network input (i.e., the spike of the $p_{m}^{\text {th }}$ neuron), we must have $\tau_{p_{m+1} q_{m+1}}=\tau_{p_{m} q_{m}}$ and also $\tilde{\tau}_{p_{m+1} q_{m+1}}=\tilde{\tau}_{p_{m} q_{m}}$ for sufficiently small $\epsilon$. Therefore we obtain $\tilde{\tau}_{p_{m+1} q_{m+1}}=\tau_{p_{m+1} q_{m+1}}$. If the spike of the $p_{m+1}^{\text {th }}$ neuron is caused by the feedforward input, we still have $\tilde{\tau}_{p_{m+1}, q_{m+1}}=\tau_{p_{m+1}, q_{m+1}}$ since the reference and perturbed trajectories receive the same feedforward input. Therefore, equation (5.4) always holds, and we obtain that $\tau_{j k}=\tilde{\tau}_{j k}$ in equations (2.1), (2.3), and (5.3).

As a result of the discussion in the preceding paragraphs, we conclude that the time evolution of the perturbation, $\delta \boldsymbol{v}(t)=\tilde{\boldsymbol{v}}(t)-\boldsymbol{v}(t)$, can be obtained from the system of equations

$$
\frac{d}{d t} \delta v_{i}(t)=-g_{L} \delta v_{i}(t)
$$

It is now easy to see that the largest Lyapunov exponent is always negative in equation (5.5).

Incidentally, the largest Lyapunov exponent approaches negative infinity because the voltage of both the reference and perturbed trajectories of any neuron (say $j^{\text {th }}$ neuron) will be reset to $V_{R}$ after its firing and will no longer separate from one-another. Therefore, the difference of the reference and perturbed trajectories of the $j^{\text {th }}$ neuron will converge together after its first firing event.

\section{Discussion and conclusions}

Oscillations in neuronal networks present a vast subject of intense current research, both theoretical and experimental $[8,9,18,19,28,30,31,34,38,48,64,76,79$, $80,85,89,90]$. They represent a number of fundamental rhythms in the brains of vertebrates $[45,51,89]$ and insects $[45,66]$. These rhythms are conjectured to function as "clocks" and means of information encoding for various brain areas, yet, it is not 
completely clear whether this is indeed the case or whether they are mere epiphenomenon [80]. Clearly, this paper cannot even begin to address the form or function of all types of neuronal network oscillations, or analyze all possible mechanisms that may cause them.

Instead, we have focused on the oscillation mechanism in the oldest and most basic version of a neuronal network model, the all-excitatory, current-based, all-to-all coupled, I\&F network, with instantaneous injected currents. We found robust oscillations even in the case when each neuron in the network is driven by a random Poisson-distributed train of external spikes. While the mechanism that sustains the oscillations is clearly identified, even in this simplest model the mathematical solutions describing it are only approximate. These approximations include the diffusion approximation in the KFE in section 3.2.2, the cascade-susceptibility condition in section 3.1.3, the assumption that no neuronal voltage reenters the interval $\left[V_{R}, V_{T}\right]$ before all the neurons have fired in section 3.1.2, the Gaussian form of the voltage PDF in section 3.1.1, and the Poisson nature of the train of the network spikes in section 4. These approximations apply to both the oscillatory and steady solutions. More accurate solutions are not currently available, with the exception of the stationary voltage PDF, which was found for the differential-difference KFE in [82] for a single (i.e. uncoupled) neuron. Nevertheless, comparison with numerical simulations shows that the approximations we made are very accurate and we still capture many phenomena of the network dynamics.

We emphasize that the main characteristic of the oscillations, their period, is always determined by fluctuations of the voltage and much less by its mean, which are determined by the respective variance and mean of the external driving force, as discussed in section 3. The voltage PDF mean does enter the description in that it is driven towards a value past the firing threshold in the superthreshold and a value below it in the subthreshold driving regime, which both increases the importance of the fluctuations in the latter regime and prompts the use of different mathematical techniques in the two regimes. Yet, in both cases it is the first neuronal voltage to reach threshold that triggers a total firing event. This is in strong contrast to the asynchronous dynamics. There, for the stationary solution deep into the mean-driven regime, the mean external driving force clearly determines the firing rate and its fluctuations have no influence, as discussed in section 4.4.

Finally, our proof in section 5 that chaotic dynamics are absent from the type of networks investigated in this paper points to the regular nature of the attractors that such networks possess. Our numerical computations show many regimes in which near-synchronized oscillations take place in addition to the regimes supporting synchronization via total firing events. This poses the broad question of what precisely is the attractor of an I\&F nework, and what bifurcations it undergoes as the network parameters change. In particular, on the one hand, chaos is absent even from conductance-based I\&F networks as long as their conductances act instantaneously or else on very short time scales, yet it is present in such systems in moderate network coupling regimes if the conductance time scale is sufficiently long. Presenting a coherent picture of the attractors for I\&F networks thus clearly furnishes a challenging theoretical problem that we intend to explore in the future.

Appendix A. Associated stationary distribution. We note that the problem of interest, equation (3.53) with boundary conditions (3.56), does not have a nontrivial stationary distribution. The stationary distribution for the same partial differential equation with reflecting boundary conditions (zero flux) at both ends of the domain 
$\left[V_{R}, V_{T}\right]$ is found by integrating

$$
0=\frac{d}{d x}\left[\left(g_{L}\left(x-V_{R}\right)-f \nu\right) p_{s}(x)\right]+\frac{f^{2} \nu}{2} \frac{d^{2}}{d x^{2}} p_{s}(x)
$$

once, which results in

$$
c=\left(g_{L}\left(x-V_{R}\right)-f \nu\right) p_{s}(x)+\frac{f^{2} \nu}{2} \frac{d}{d x} p_{s}(x) .
$$

For the reflecting boundary conditions, the constant, $c$, must be zero. Integrating once more we obtain the stationary distribution,

$$
p_{s}(x)=\mathcal{N} \exp \left(-\frac{\left(g_{L}\left(x-V_{R}\right)-f \nu\right)^{2}}{f^{2} \nu g_{L}}\right),
$$

where the constant $\mathcal{N}$ would normally be chosen so that $p_{s}(x)$ integrates to one over the domain. However, our use of the stationary distribution to transform the equations for the eigenfunctions, $P_{n}(x)$, does not rely on it being properly normalized, so we choose $\mathcal{N}=1$ for simplicity. If we shift and rescale the variable $x$ so that

$$
z=\frac{g_{L}\left(x-V_{R}\right)-f \nu}{f \sqrt{g_{L} \nu}},
$$

the stationary distribution has the compact form

$$
\tilde{p}_{s}(z)=\tilde{\mathcal{N}} e^{-z^{2}} .
$$

In terms of the variable $z$, the system is driven to $z=0$ by the average drift, and $x$ is essentially rescaled by the standard deviation.

Appendix B. Simultaneous solution of KFE and KBE. Here we show how to simultaneously find the solution of the KFE (3.53) with the boundary conditions (3.56) and the corresponding $\mathrm{KBE}$

$$
\frac{\partial}{\partial t} q(x, t)=\left[-g_{L}\left(x-V_{R}\right)+f \nu\right] \frac{\partial}{\partial x} q(x, t)+\frac{f^{2} \nu}{2} \frac{\partial^{2}}{\partial x^{2}} q(x, t),
$$

with the adjoint boundary conditions

$$
\left.\frac{\partial}{\partial x} q(x, t)\right|_{x=V_{R}}=0
$$

and

$$
q\left(V_{T}, t\right)=0 .
$$

In particular, the solution $p_{v}(x, t)$, can be written in terms of the solution $q(x, t)$ to the KBE problem (B.1) as [35, Sec. 5.2.5],

$$
p_{v}(x, t)=p_{s}(x) q(x, t),
$$

where $p_{s}(x)$ is the stationary solution to the KFE (3.53) with reflecting boundary conditions

$$
J\left[p_{s}\right]\left(V_{R}\right)=J\left[p_{s}\right]\left(V_{T}\right)=0 .
$$


The stationary distribution, equation (A.3), is derived in appendix A.

This result is especially useful because it implies that only one set of eigenfunctions from the two eigenvalue problems, (3.59) and (3.63), is needed to form the solution to (3.53). Also, it suggests an equivalent self adjoint form which will be derived later. Due to its importance, we show this result in a slightly more general form. In particular, we consider a general $\mathrm{KFE}$ of the form

$$
\frac{\partial}{\partial t} p(x, t)=-\frac{\partial}{\partial x}(A(x) p(x, t))+B \frac{\partial^{2}}{\partial x^{2}} p(x, t),
$$

and corresponding equation for the steady state probability density function

$$
0=-\frac{d}{d x}\left(A(x) p_{s}(x)\right)+B \frac{d^{2}}{d x^{2}} p_{s}(x),
$$

where $A(x)$ is an arbitrary smooth function of $x$ and $B$ is a constant. Equation (B.5) can be integrated once, and with reflecting boundary conditions (zero flux for only the steady state solution) simplifies to

$$
0=-A(x) p_{s}(x)+B \frac{d}{d x} p_{s}(x) .
$$

We now take the ansatz $p_{s}(x) q(x, t)$ and insert it into equation (B.4) for $p(x, t)$ to obtain an equation for $q(x, t)$, which reads

$$
\begin{aligned}
p_{s} \frac{\partial}{\partial t} q & =-A p_{s} \frac{\partial}{\partial x} q-q \frac{\partial}{\partial x}\left(A p_{s}\right)+B\left(p_{s} \frac{\partial^{2}}{\partial x^{2}} q+2 \frac{\partial}{\partial x} p_{s} \frac{\partial}{\partial x} q+q \frac{\partial^{2}}{\partial x^{2}} p_{s}\right) \\
& =q\left[-\frac{\partial}{\partial x}\left(A p_{s}\right)+B \frac{\partial^{2}}{\partial x^{2}} p_{s}\right]-A p_{s} \frac{\partial}{\partial x} q+B\left(p_{s} \frac{\partial^{2}}{\partial x^{2}} q+2 \frac{\partial}{\partial x} p_{s} \frac{\partial}{\partial x} q\right)
\end{aligned}
$$

where the explicit dependence on $x$ and $t$ has been suppressed. The expression on the last line in brackets vanishes because of equation (B.6), which also makes it possible to replace the last term by $2 A p_{s} \partial q / \partial x$. After canceling the factor $p_{s}$, which does not vanish, we find for the function $q(x, t)$ the equation

$$
\frac{\partial}{\partial t} q(x, t)=A(x) \frac{\partial}{\partial x} q(x, t)+B \frac{\partial^{2}}{\partial x^{2}} q(x, t),
$$

which is exactly the KBE (adjoint operator) for the given KFE. We note that no assumptions on the boundary condition of $p_{v}(x, t)$ nor $q(x, t)$ have been made, only that $p_{s}(x)$ has reflecting boundary conditions.

The requirements of the boundary conditions come into play in the orthogonality of the eigenfunctions. At this point, let us investigate the case when $p_{v}(x, t)=$ $P_{n}(x) e^{-\lambda_{n} t}$ and $q(x, t)=Q_{m}(x) e^{-\mu_{m} t}$, where $P_{n}(x)$ and $Q_{m}(x)$ solve their corresponding eigenvalue problems. Consider the inner product of the two eigenfunctions,

$$
\begin{aligned}
&\left(\mu_{m}-\lambda_{n}\right) \int_{a}^{b} P_{n}(x) Q_{m}(x) d x=\int_{a}^{b}-\frac{d}{d x}\left(A P_{n}\right) Q_{m}-A P_{n} \frac{d}{d x} Q_{m} \\
&+B\left[Q_{m} \frac{d^{2}}{d x^{2}} P_{n}-P_{n} \frac{d^{2}}{d x^{2}} Q_{m}\right] d x .
\end{aligned}
$$

Integrating by parts, and simplifying, we obtain the following conditions:

$$
\left[-A P_{n}+B \frac{d}{d x} P_{n}\right]_{x=V_{R}} Q_{m}\left(V_{R}\right)-\left.B P_{n}\left(V_{R}\right) \frac{d}{d x} Q_{m}\right|_{x=V_{R}}=0
$$


and

$$
\left[-A Q_{m}-B \frac{d}{d x} Q_{m}\right]_{x=V_{T}} P_{n}\left(V_{T}\right)+\left.B \frac{d}{d x} P_{n}\right|_{x=V_{T}} Q_{m}\left(V_{T}\right)=0 .
$$

In equation (B.10), the probability flux generated by $P_{n}(x)$ vanishes at $V_{R}$ by assumption, thus for this equation to be satisfied the requirement on $Q_{m}(x)$ is

$$
\left.\frac{d}{d x} Q_{m}(x)\right|_{x=V_{R}}=0 .
$$

In equation (B.11), the function $P_{n}(x)$ vanishes at $V_{T}$, which we assumed for our KFE eigenvalue problem (3.59) thus for this equation to be satisfied the requirement on the eigenfunction $Q_{m}(x)$ is

$$
Q_{m}\left(V_{T}\right)=0 .
$$

These are precisely the boundary conditions (3.63b) and (3.63c). Thus, the problem is reduced to finding the stationary solution $p_{s}(x)$ and and the eigenfunctions $Q_{m}(x)$.

The solution to the KFE (3.53) with boundary conditions (3.56) can now be written in the form $p_{v}(x, t)=p_{s}(x) q(x, t)$, where $q(x, t)$ is the solution of the KBE with the corresponding boundary conditions, (B.1). This suggests that there exists a self-adjoint operator for which $w(x, t)=p_{v}(x, t) p_{s}(x)^{-1 / 2}=q(x, t) p_{s}(x)^{1 / 2}$ is the solution. To find this operator we follow the standard symmetrization procedure for stochastic systems with detailed balance [40, Sec. 4.7]. We begin by substituting $p_{v}(x, t)=p_{s}(x)^{1 / 2} w(x, t)$ into equation (3.53). Using the non-normalized stationary distribution from equation (3.61), the resulting equation for $w(x, t)$ is

$$
\frac{\partial}{\partial t} w(x, t)=\frac{f^{2} \nu}{2} \frac{\partial^{2}}{\partial x^{2}} w(x, t)+\frac{g_{L}}{2}\left(1-\frac{\left(g_{L}\left(x-V_{R}\right)-f \nu\right)^{2}}{f^{2} g_{L} \nu}\right) w(x, t) .
$$

This equation is self adjoint, with a compact evolution operator, therefore the eigenfunctions of both this and the original problem form an orthonormal complete set with the same spectrum of eigenvalues. This symmetrization procedure works broadly for Markov processes with detailed balance [40, Sec. 4.7]. 
Appendix C. Computational details of obtaining the solution. In this section the solutions to various numerical difficulties encountered while evaluating the solution to the KFE (3.53) are presented. First we discuss why different sets of basis functions are useful for computing at different stages of the solution process. The next discussion is related to the numerical evaluation of the confluent hypergeometric function. Last, a different form for the flux at the boundary is presented in terms of the series expansion, equation (3.62).

C.1. Choice of basis functions. The solution to equation (3.69) can be written in various forms. Any two linearly independent forms could be taken as the basis for the solution. Various difficulties are encountered depending on the choice.

The solutions ${ }_{1} F_{1}(a, b, \zeta)$ and $\zeta^{1-b}{ }_{1} F_{1}(a+1-b, b+1, \zeta)$ both grow exponentially for large $\zeta$ and negative $a$. Recalling that the left boundary in the physical variable $x$ is a large positive number in the transformed variable $\zeta$, in order to satisfy the left boundary condition (zero derivative) the linear combination must cancel this exponential growth. Numerically this is difficult to compute accurately, so selecting a basis where one function does not contain exponential growth is better. This function is $U(a, b, \zeta)$, which grows like $\zeta^{-a}$ for large $\zeta$ (recall that for our purposes $a<0$ ). The basis ${ }_{1} F_{1}(a, b, \zeta)$ and $U(a, b, \zeta)$ is used for $|a|<52$; the large (negative) $a$ asymptotics for the basis ${ }_{1} F_{1}(a, b, \zeta)$ and $\zeta^{1-b}{ }_{1} F_{1}(a+1-b, b+1, \zeta)$ are better suited for numerical evaluation.

The basis of ${ }_{1} F_{1}(a, b, \zeta)$ and $U(a, b, \zeta)$ is not a good choice in evaluating the determinant and finding eigenvalues. There are many zeros to the resulting equation which do not correspond to eigenvalues of the problem. These extraneous zeros lead to "eigenfunctions" which do not satisfy the required boundary and matching conditions. Therefore, it is better to use the basis ${ }_{1} F_{1}(a, b, \zeta)$ and $\zeta^{1-b}{ }_{1} F_{1}(a+1-b, b+1, \zeta)$ in order to determine the eigenvalues. This basis causes the determinant equation to have vertical asymptotes with one eigenvalue between each consecutive set of asymptotes. The basis of ${ }_{1} F_{1}(a, b, \zeta)$ and $U(a, b, \zeta)$ also has asymptotes, but may have one or more crossings between consecutive sets of asymptotes, none of which are guaranteed to be eigenvalues.

C.2. Asymptotic forms and recursion relations of ${ }_{1} F_{1}$. For many values of $a$ and $x$ the infinite series representation of ${ }_{1} F_{1}$, equation (3.70), is either slow to converge or introduces dynamic range error. Often this occurs for large values of $a$ and $x$, where asymptotic relations are available for computational use instead. When evaluating between two asymptotic regions, recursion relations are used to move the parameters of ${ }_{1} F_{1}$ into the asymptotic region. Various asymptotics and recursion relations which are used to compute ${ }_{1} F_{1}$ are given.

When only $x$ is large and $a$ and $b$ are fixed, the confluent hypergeometric function can be approximated by a truncated series given in [2]. For real $x$, this series is

$$
\frac{{ }_{1} F_{1}(a, b, x)}{\Gamma(b)}=\frac{e^{x} x^{a-b}}{\Gamma(a)}\left[\sum_{n=0}^{S-1} \frac{(b-a)_{n}(1-a)_{n}}{n !} x^{-n}+O\left(|x|^{-S}\right)\right] .
$$

A finite number of terms are used such that the relative error of the function is less then $O\left(10^{-10}\right)$. For large eigenvalues, the confluent hypergeometric function is evaluated at large negative values of $a$. For these large real values of $a$ and $x$, asymptotic approximations [83, Sec. 4.5] are used for the confluent hypergeometric function, ${ }_{1} F_{1}(a, b, x)$. These asymptotics are valid inside wedges in the $x-k$ plane, 


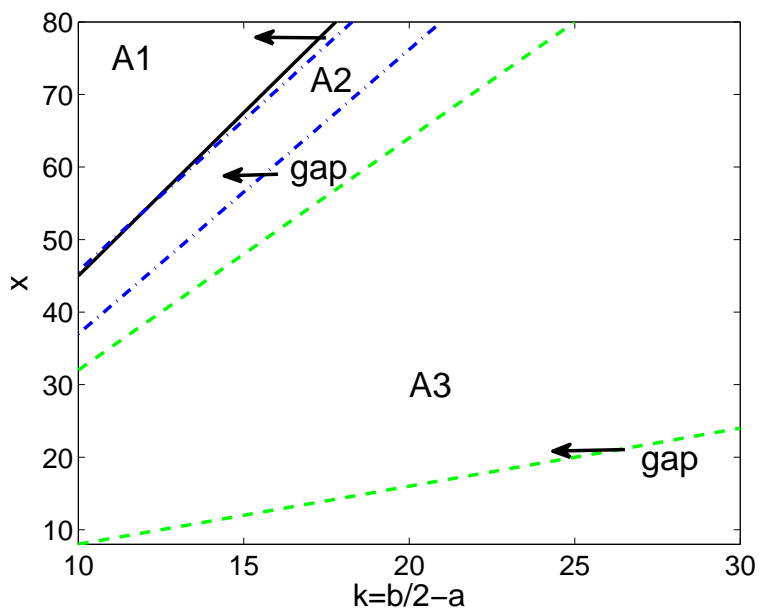

FIG. C.1. Regions in the $k-x$ plane where the asymptotic expansions of ${ }_{1} F_{1}$ are valid. Between regions of validity, recursion relation (C.2) is used to move evaluation in the direction indicated. Within each region, the corresponding equations are: A1 - equation (C.3), A2 - equation (C.4) and A3 - equation (C.11)

where $k=b / 2-a$, depicted in figure C.1. As the wedges do not overlap, in between each region the recurrence relation [2]

$$
(b-a)_{1} F_{1}(a-1, b, x)+(2 a-b+x)_{1} F_{1}(a, b, x)-a_{1} F_{1}(a+1, b, x)=0
$$

is used to send the evaluation of the function to smaller values of $a$. Wedges are selected so that the resulting function evaluation formed a close match to Mathematica's Hypergeometric1F1 function. We proceed to define the wedges and the asymptotic form within each wedge.

The first wedge is defined for $x \geq(1+\eta) 4 k$. We define $\sqrt{x / 4 k}=\cosh \theta$ and write the asymptotic form of the confluent hypergeometric functions as [83, Sec. 4.5]

$$
\begin{aligned}
{ }_{1} F_{1}(a, b, x)=\Gamma(b) \sin a \pi \exp [ & \left.2 k\left(\frac{1}{2} \sinh 2 \theta-\theta+\cosh ^{2} \theta\right)\right] \\
\times & \frac{(2 k \cosh \theta)^{1-b}}{\sqrt{\pi k \sinh 2 \theta}}\left(1+O\left(k^{-1}\right)\right) .
\end{aligned}
$$

For the next wedge, $4 k \approx x$, we specify the region where this approximation is valid by first defining $t$ in terms of $x$ by

$$
x=4 k-2\left(\frac{2 k}{3}\right)^{1 / 3} t
$$

The wedge includes $x$ and $k$ such that $-1.5 \leq t \leq 0.8$. For the asymptotics, we define $\alpha=(5 b-2) / 10, \beta=\Gamma(1 / 3) / 2 \Gamma(2 / 3)$ and $t^{\prime}=-3^{-1 / 3} t$. The confluent hypergeometric 
function is then written in the form [83, Sec. 4.5.1]

$$
\begin{aligned}
\frac{e^{-x / 2}}{\Gamma(b)}{ }_{1} F_{1}(a, b, x)=\gamma_{1}[ & \operatorname{Ai}\left(t^{\prime}\right)+3^{-1 / 3}\left(\frac{t^{2}}{5} \operatorname{Ai}^{\prime}\left(t^{\prime}\right)+\alpha(t-\beta) \operatorname{Ai}\left(t^{\prime}\right)\right. \\
& \left.\left.+\alpha \beta 3^{-1 / 2} \operatorname{Bi}\left(t^{\prime}\right)\right)(2 k)^{-2 / 3}+O\left(k^{-4 / 3}\right)\right] \\
+\gamma_{2}[ & \operatorname{Bi}\left(t^{\prime}\right)+3^{-1 / 3}\left(\frac{t^{2}}{5} \operatorname{Bi}^{\prime}\left(t^{\prime}\right)+\alpha(t+\beta) \operatorname{Bi}\left(t^{\prime}\right)\right. \\
& \left.\left.-\alpha \beta 3^{-1 / 2} \operatorname{Ai}\left(t^{\prime}\right)\right)(2 k)^{-2 / 3}+O\left(k^{-4 / 3}\right)\right],
\end{aligned}
$$

where the coefficients are given by

$$
\gamma_{1}=\frac{1}{3^{1 / 3}(2 k)^{b-2 / 3}}\left[3^{1 / 3} \cos a \pi+2 \alpha \beta \frac{\sin (a \pi+\pi / 6)}{(2 k)^{2 / 3}}+O\left(k^{-4 / 3}\right)\right]
$$

and

$$
\gamma_{2}=\frac{1}{3^{1 / 3}(2 k)^{b-2 / 3}}\left[3^{1 / 3} \sin a \pi-2 \alpha \beta \frac{\sin (a \pi+\pi / 6)}{3^{1 / 2}(2 k)^{2 / 3}}+O\left(k^{-4 / 3}\right)\right] .
$$

The functions $\operatorname{Ai}(x)$ and $\operatorname{Bi}(x)$ are the Airy functions [2], which are linearly independent solutions to the differential equation

$$
\frac{d^{2}}{d x^{2}} w(x)-x w(x)=0
$$

The Airy functions can be written in terms of two other functions, $f(x)$ and $g(x)$, as

$$
\operatorname{Ai}(x)=c_{1} f(x)-c_{2} g(x) \quad \text { and } \quad \operatorname{Bi}(x)=\sqrt{3}\left(c_{1} f(x)+c_{2} g(x)\right)
$$

where

$$
f(x)=\sum_{k=0}^{\infty} 3^{k}\left(\frac{1}{3}\right)_{k} \frac{z^{3 k}}{(3 k) !} \quad \text { and } \quad g(x)=\sum_{k=0}^{\infty} 3^{k}\left(\frac{2}{3}\right)_{k} \frac{z^{3 k+1}}{(3 k+1) !}
$$

with

$$
c_{1}=\operatorname{Ai}(0)=\frac{3^{-2 / 3}}{\Gamma(2 / 3)} \quad \text { and } \quad c_{2}=-\mathrm{Ai}^{\prime}(0)=\frac{3^{-1 / 3}}{\Gamma(1 / 3)} .
$$

The last wedge is given by ${ }^{1}, 4 \eta k \leq x \leq(1-\eta) 4 k$. Defining

$$
\cos \theta=\sqrt{\frac{x}{4 k}} \text { and } \Theta=k(2 \theta-\sin 2 \theta)+\pi / 4,
$$

the asymptotics are [83, Sec. 4.5.2]

$$
\begin{aligned}
\frac{{ }_{1} F_{1}(a, b, x)}{\Gamma(b)}= & \frac{\exp \left(2 k \cos ^{2} \theta\right)(2 k \cos \theta)^{1-b}}{(\pi k \sin 2 \theta)^{1 / 2}} \\
& \times\left\{\sin (\Theta+a \pi)-A_{1}(\theta) \frac{\cos (\Theta+a \pi)}{k \sin 2 \theta}+O\left(k^{-2}\right)\right\}
\end{aligned}
$$

\footnotetext{
${ }^{1}$ missing 4 in [83], $\eta k$ replaced by $4 \eta k$
} 
where

$$
A_{1}(\theta)=\frac{1}{12}\left\{\frac{5}{4 \sin ^{2} \theta}+\left(3 b^{2}-6 b+2\right) \sin ^{2} \theta-1\right\} .
$$

For small eigenvalues $\left(\lambda_{n} \ll 1\right)$, the confluent hypergeometric function is evaluated at small negative values of $a$. In this region, when $x$ is also small to moderate size, the series representation is slow to converge. For this reason, the recurrence relation (C.2) is used to send evaluation to larger values of $a$.

C.3. Numerical evaluation of the flux at the boundary. In order to obtain the PDF of the first exit time, equation (3.44), the voltage probability flux at the upper boundary, $V_{T}$, is required. To avoid the error in numerically evaluating derivatives, we differentiate the terms in the sum analytically. Also, evaluating $1-$ $F_{T}(t)$ is numerically easier than evaluating $F_{T}(t)$, the CDF of the first exit time, $T$.

First we consider the form of the series expansion which will be easiest to evaluate numerically. Returning to the expression for the PDF of the exit time for a single neuron, equation (3.44):

$$
p_{T}(t)=J\left[p_{v}\right]\left(V_{T}, t\right),
$$

we write this in terms of the series expansion, equation (3.62), as

$$
\begin{aligned}
p_{T}(t)= & -\sum_{n=0}^{\infty} A_{n}\left[\left.\frac{f^{2} \nu}{2} \frac{d}{d x}\left(p_{s}(x) Q_{n}(x)\right)\right|_{x=V_{T}}\right. \\
& \left.+\left(g_{L}\left(V_{T}-V_{R}\right)-f \nu\right) p_{s}\left(V_{T}\right) Q_{n}\left(V_{T}\right)\right] e^{-\lambda_{n} t} .
\end{aligned}
$$

We denote this by

$$
p_{T}(t)=\sum_{n=0}^{\infty} A_{n} J_{n}\left(V_{T}\right) e^{-\lambda_{n} t},
$$

where both $A_{n}$ and $J_{n}\left(V_{T}\right)$ are constants which do not depend on $t$. The CDF can then be computed by integrating the PDF from 0 to $t$. Numerically, this is not in a good form to evaluate, as the infinite series decays slowly, requiring many terms to be included for an accurate evaluation. If instead, equation (C.14) is integrated from $t$ to infinity, we obtain

$$
1-F_{T}(t)=\int_{t}^{\infty} p_{T}\left(t^{\prime}\right) d t^{\prime}=\sum_{n=0}^{\infty} \frac{A_{n}}{\lambda_{n}} J_{n}\left(V_{T}\right) e^{-\lambda_{n} t},
$$

which requires inclusion of fewer terms for the same numerical accuracy.

Acknowledgement. K.A.N. was supported by an NSF Graduate Research Fellowship. P.R.K. was partly supported by NSF grant DMS-0449717. D.Z., A.V.R., and D.C. were partly supported by NSF grants DMS-0506396 and DMS-0507901, and a grant from the Swartz Foundation. G.K. was partly supported by NSF grant DMS-0506287, and gratefully acknowledges the hospitality of the Courant Institute of Mathematical Sciences. 


\section{REFERENCES}

[1] L. Abbott and C. Van Vreeswijk, Asynchronous states in networks of pulse-coupled oscillators, Phys. Rev. E, 48, 1483-1490, 1993.

[2] M. Abramowitz and I.A. Stegun, Handbook of Mathematical Functions, Dover Pulications, Inc., ninth edition, 1972.

[3] C. Allen and C.F. Stevens, An evaluation of causes for unreliability of synaptic transmission, Proc. Natl. Acad. Sci. USA, 91, 10380-10383, 1994.

[4] K.T. Alligood, T.D. Sauer and J.A. Yorke, Chaos: An Introduction to Dynamical Systems, Springer, 1997.

[5] D.J. Amit and N. Brunel, Model of global spontaneous activity and local structured activity during delay periods in the cerebral cortex, Cereb. Cortex, 7, 237-252, 1997.

[6] G. Barna, T. Grobler and P. Erdi, Statistical model of the hippocampal ca3 region, ii. the population framework: model of rhythmic activity in ca3 slice, Biol. Cybern., 79, 309321, 1998

[7] D.P. Bertsekas and J.N. Tsitsiklis, Introduction to Probability, Athena Scientific, Nashua, NH, second edition, 2008.

[8] C. Borgers, S. Epstein and N.J. Kopell, Gamma oscillations mediate stimulus competition and attentional selection in a cortical network model., Proc. Natl. Acad. Sci. USA, 105, $18,18023-18028,2008$.

[9] C. Borgers and N. Kopell, Effects of noisy drive on rhythms in networks of excitatory and inhibitory neurons, Neural Comput., 17, 557-608, 2005.

[10] R. Brette, Dynamics of one-dimensional spiking neuron models., J. Math. Biol., 48, 38-56, 2004.

[11] R. Brette, M. Rudolph, T. Carnevale, M. Hines, D. Beeman, J.M. Bower, M. Diesmann and et al, Simulation of networks of spiking neurons: a review of tools and strategies, J. Comput. Neurosci., 23, 349-398, 2007.

[12] V. Bringuier, F. Chavane, L. Glaeser and Y. Fregnac, Horizontal propagation of visual activity in the synaptic integration field of area 17 neurons, Science, 283, 695-699, 1999.

[13] N. Brunel, Dynamics of networks of randomly connected excitatory and inhibitory spiking neurons, J. Physiol. Paris, 94, 445-463, 2000.

[14] N. Brunel and V. Hakim, Fast global oscillations in networks of integrate-and-fire neurons with low firing rates, Neural Comput., 11, 1621-1671, 1999.

[15] N. Brunel and S. Sergi, Firing frequency of leaky integrate-and-fire neurons with syncaptic current dynamics, J. Theor. Bio., 195, 87-95, 1998.

[16] A.N. Burkitt, A review of the integrate-and-fire neuron model: II. Inhomogeneous synaptic input and network properties, Biol. Cybern., 95, 97-112, 2006.

[17] A.N. Burkitt, A review of the integrate-and-fire neuron model: I. Homogeneous synaptic input, Biol. Cybern., 95, 1-19, 2006.

[18] P. Buser and A. Rougeul-Buser, Do cortical and thalamic bioelectric oscillations have a functional role? a brief survey and discussion., J. Physiol. Paris, 89, 249-254, 1995.

[19] G. Buzsaki and A. Draguhn, Neuronal oscillations in cortical networks, Science, 25, 304, 1926-1929, 2004.

[20] D. Cai, A. Rangan and D. McLaughlin, Architectural and synaptic mechanisms underlying coherent spontaneous activity in V1, Proc. Nat'l Acad. Sci. (USA), 102, 5868-5873, 2005.

[21] D. Cai, L. Tao, A.V. Rangan and D.W. McLaughlin, Kinetic theory for neuronal network dynamics, Commun. Math. Sci., 4, 97-127, 2006.

[22] D. Cai, L. Tao, M. Shelley and D. McLaughlin, An effective representation of fluctuationdriven neuronal networks with application to simple 83 complex cells in visual cortex, Pro. Nat. Acad. Sci. (USA), 101, 7757-7762, 2004.

[23] A. Casti, A. Omurtag, A. Sornborger, E. Kaplan, B. Knight, J. Victor and L. Sirovich, A population study of integrate-and-fire-or-burst neurons, Neural Comp., 14, 957-986, 2002.

[24] T. Chawanya, A. Aoyagi, T. Nishikawa, K. Okuda and Y. Kuramoto, A model for feature linking via collective oscillations in the primary visual cortex, Biol. Cybern., 68, 483-90, 1993.

[25] E. Cinlar, Superposition of point processes, Stochastic Point Processes: Statistical Analysis, Theory, and Applications, P. Lewis (ed.), Wiley, New York, NY, 549-606, 1972.

[26] R.L. DeVille and C.S. Peskin, Synchrony and asynchrony in a fully stochastic neural network, Bull. Math. Bio., 70, 1608-1633, 2008.

[27] P. Diţă, The Fokker-Planck Equation with absorbing boundary, J. Phys. A: Math. Gen., 18, 2685-2690, 1985. 
[28] R. Eckhorn, Oscillatory and non-oscillatory synchronizations in the visual cortex and their possible roles in associations of visual features, Prog. Brain Res., 102, 405-426, 1994.

[29] R. Eckhorn, A.M. Gail, A. Bruns, A. Gabriel, B. Al-Shaikhli and M. Saam, Different types of signal coupling in the visual cortex related to neural mechanisms of associative processing and perception, IEEE Trans. Neural Netw., 15, 1039-1052, 2004.

[30] A. K. Engel, P. Fries and W. Singer, Dynamic predictions: oscillations and synchrony in top-down processing, Nat. Rev. Neurosci, 2, 704-716, 2001.

[31] G.B. Ermentrout and N. Kopell, Fine structure of neural spiking and synchronization in the presence of conduction delays, Proc. Natl. Acad. Sci. USA, 95, 3, 1259-1264, 1998.

[32] W. Feller, An Introduction to Probability Theory and Its Applications, John Wiley, New York, 1968.

[33] N. Fourcaud and N. Brunel, Dynamics of the firing probability of noisy integrate-and-fire neurons, Neural Comp., 14, 2057-2110, 2002.

[34] P. Fries, D. Nikolic and W. Singer, The gamma cycle, Trends Neurosci., 30, 309-316, 2007.

[35] C.W. Gardiner, Handbook of Stochastic Methods, Springer, third edition, 2004.

[36] W. Gerstner, Population dynamics of spiking neurons: fast transients, asynchronous states, and locking, Neural Comput., 12, 43-89, 2000.

[37] W. Gerstner and W. Kistler, Spiking Neuron Models - Single Neurons, Populations, Plasticity, Cambridge University Press, New York, 2002.

[38] C.M. Gray, Synchronous oscillations in neuronal systems: mechanisms and functions, J. Comput. Neurosci., 1, 11-38, 1994.

[39] J. Guckenheimer and R.A. Oliva, Chaos in the Hodgkin-Huxley model, SIAM Journal on Applied Dynamical Systems, 1(1), 105-114, 2002.

[40] H. Haken, Synergetics: an Introduction, Nonequilibrium phase transitions and selforganization in physics, chemistry, and biology, Springer-Verlag, Berlin, third edition, 1983.

[41] N.R. Hardingham and A. U. Larkman, The reliability of excitatory synaptic transmission in slices of rat visual cortex in vitro is temperature dependent, J. Physiol., 507, 249-256, 1998.

[42] E. Haskell, D. Nykamp and D. Tranchina, Population density methods for large-scale modeling of neuronal networks with realistic synaptic kinetics: cutting the dimension down to size, Network: Compt. Neural. Syst., 12, 141-174, 2001.

[43] A.L. Hodgkin and A.F. Huxley, A quantitative description of membrane current and its application to conduction and excitation in nerve, J. Physiol. (Lond.), 117, 500-544, 1952.

[44] S. Karlin and H.M. Taylor, A First Course in Stochastic Processes, Boston, second edition, 1975.

[45] L.M. Kay and M. Stopfer, Information processing in the olfactory systems of insects and vertebrates, Semin Cell Dev Biol, 17, 433-442, 2006.

[46] B. Knight, Dynamics of encoding in a populaton neurons, J. Gen. Physiol., 59, 734-766, 1972.

[47] C. Koch, Biophysics of Computation, Oxford University Press, Oxford, 1999.

[48] N. Kopell, G.B. Ermentrout, M.A. Whittington and R.D. Traub, Gamma rhythms and beta rhythms have different synchronization properties, Proc. Natl. Acad. Sci. USA, 97(15), $1867-1872,2000$.

[49] G. Kovačič, L. Tao, A.V. Rangan and D. Cai, Fokker-Planck description of conductance-based integrate-and-fire neuronal networks, Phys. Rev. E, 80, 021904, 2009.

[50] Y. Kuramoto, Collective synchronization of pulse-coupled oscillators and excitable units, Physica D (Netherlands), 50, 15-30, 1991/05/.

[51] F. Laberge and T.J. Hara, Neurobiology of fish olfaction: a review, Brain Res Brain Res Rev, $36,46-59,2001$.

[52] L. Lapicque, Recherches quantitatives sur l'excitation electrique des nerfs traitee comme une polarization, Journal de Physiologie et Pathologie Géné ral, 9, 620-635, 1907.

[53] B. Lindner, Superposition of many independent spike trains is generally not a poisson process, Physical Review E (Statistical, Nonlinear, and Soft Matter Physics), 73, $022901,2006$.

[54] D. McLaughlin, R. Shapley, M. Shelley and J. Wielaard, A neuronal network model of macaque primary visual cortex (V1): orientation selectivity and dynamics in the input layer $4 C \alpha$, Proc. Natl. Acad. Sci. USA, 97, 8087-8092, 2000.

[55] R.E. Mirollo and S.H. Strogatz, Synchronization of pulse-coupled biological oscillators, SIAM J. Appl. Math., 50, 1645-1662, 1990.

[56] P.L. Nunez, Neocortical Dynamics and Human EEG Rhythms, Oxford University Press, New York, Oxford, 1995. 
[57] D. Nykamp and D. Tranchina, A population density method that facilitates large-scale modeling of neural networks: analysis and application to orientation tuning, J. Comput. Neurosci., 8, 19-50, 2000.

[58] P.L. Nunez, A population density method that facilitates large-scale modeling of neural networks: extension to slow inhibitory synapses, Neural Comput., 13, 511-546, 2001.

[59] B. Øksendal, Stochastic Differential Equations, Universitext, Springer-Verlag, Berlin, fifth ed., 1998.

[60] A. Omurtag, E. Kaplan, B. Knight and L. Sirovich, A population approach to cortical dynamics with an application to orientation tuning, Network, 11, 247-260, 2000.

[61] A. Omurtag, B. Knight and L. Sirovich, On the simulation of large populations of neurons, J. Comput. Neurosci., 8, 51-63, 2000.

[62] N. Otmakhov, A.M. Shirke and R. Malinow, Measuring the impact of probabilistic transmission on neuronal output, Neuron, 10, 1101-1111, 1993.

[63] D. Pare, E. Shink, H. Gaudreau, A. Destexhe and E. Lang, Impact of spontaneous synaptic activity on the resting properties of cat neocortical pyramidal neurons in vivo, J. Neurophysiol, 79, 1450-1460, 1998.

[64] G. Pareti and A. De Palma, Does the brain oscillate? The dispute on neuronal synchronization., Neurol Sci., 25, 41-47, 2004.

[65] T.S. Parker and L.O. Chua, Practical Numerical Algorithms for Chaotic Systems, Springer, 1989

[66] M. Patel, A.V. Rangan and D. Cai, A large-scale model of locust antennal lobe, submitted.

[67] C.S. Peskin, Mathematical Aspects of Heart Physiology, Courant Institute of Mathematical Sciences, New York, 268-278, 1975.

[68] J. Pham, K. Pakdaman, J. Champagnat and J. Vibert, Activity in sparsely connected excitatory neural networks: effect of connectivity neural networks, Neural Networks, 11 , 415-434, 1998.

[69] S.J. Pyott and C. Rosenmund, The effects of temperature on vesicular supply and release in autaptic cultures of rat and mouse hippocampal neurons, J. Physiol., 539, 523-535, 2002.

[70] A.V. Rangan and D. Cai, Maximum-entropy closures for kinetic theories of neuronal network dynamics, Phys. Rev. Lett., 96, 178101, 2006.

[71] A.V. Rangan, D. Cai and D.W. McLaughlin, Modeling the spatiotemporal cortical activity associated with the line-motion illusion in primary visual cortex, Proc. Natl. Acad. Sci. USA, 102, 18793-18800, 2005.

[72] A.V. Rangan, G. Kovačič and D. Cai, Kinetic theory for neuronal networks with fast and slow excitatory conductances driven by the same spike train, Physical Review E, 77, 041915, 2008.

[73] A.V. Rangan, L. Tao, G. Kovačič and D. Cai, Large-scale computational modeling of the primary visual cortex, K. Josić, M. Matias, R. Romo and J. Rubin (eds.), Coherent Behavior, Neuronal Networks, Springer-Verlag, 2008.

[74] S. Redman, Quantal analysis of synaptic potentials in neurons of the central nervous system, Physiological Reviews, 70, 165-198, 1990.

[75] H. Risken, The Fokker-Planck equation, Springer-Verlag, Berlin, second edition, 1989. Methods of solution and applications.

[76] R. Ritz and T.J. Sejnowski, Synchronous oscillatory activity in sensory systems: new vistas on mechanisms, Curr. Opin. Neurobiol, 7, 536-546,1997.

[77] S. Sato, Evaluation of the first-passage time probability to a square root boundary for the Wiener process, J. Applied Probability, 14, 850-856, 1977.

[78] S. Sato, On the moments of the firing interval of the diffusion approximated model neuron, Mathematical Biosciences, 39, 53-70, 1978.

[79] K. Sauve, Gamma-band synchronous oscillations: recent evidence regarding their functional significance., Conscious Cogn, 8, 213-224, 1999.

[80] T.J. Sejnowski and O. Paulsen, Network oscillations: emerging computational principles, J. Neurosci, 26(8), 1673-1676, 2006.

[81] W. Senn and R. Urbanczik, Similar nonleaky integrate-and-fire neurons with instantaneous couplings always synchronize, SIAM J. Appl. Math., 61, 1143-1155, 2000.

[82] L. Sirovich, A. Omurtag and B.W. Knight, Dynamics of neuronal populations: the equilibrium solution, SIAM J. Appl. Math., 60, 2009-2028, 2000.

[83] L.J. Slater, Confluent hypergeometric functions, Cambridge University Press, New York, 1960.

[84] D. Somers, S. Nelson and M. Sur, An emergent model of orientation selectivity in cat visual cortical simple cells, J. Neuroscience, 15, 5448-5465, 1995.

[85] M. Steriade, Grouping of brain rhythms in corticothalamic systems, Neuroscience, 137, 10871106, 2006. 
[86] Y. Sun, D. Zhou, A.V. Rangan and D. Cai, Low dimensionality and chaos in Hodgkin-Huxley neuronal network dynamics, submitted, 2008.

[87] Y. Sun, D. Zhou, A.V. Rangan and D. Cai, Pseudo-Lyapunov exponents and predictability of Hodgkin-Huxley neuronal network dynamics, submitted, 2008.

[88] L. Tao, M. Shelley, D. McLaughlin and R. Shapley, An egalitarian network model for the emergence of simple and complex cells in visual cortex, Proc. Natl. Acad. Sci. USA, 101, 366-371, 2004.

[89] R. Traub, J. Jeffreys and M. Whittington, Fast Oscillations in Cortical Circuits, MIT press, Cambridge, MA, 1999.

[90] R.D. Traub, J.G. Jefferys and M.A. Whittington, Functionally relevant and functionally disruptive (epileptic) synchronized oscillations in brain slices., Adv Neurol, 79, 709-724, 1999.

[91] H.C. Tuckwell, Introduction to Theoretical Neurobiology: Volume 1, Linear Cable Theory and Dendritic Structure, Cambridge Studies in Mathematical Biology, Cambridge University Press, 1988.

[92] H.C. Tuckwell, Introduction to Theoretical Neurobiology: Volume 2, Nonlinear and Stochastic Theories, Cambridge Studies in Mathematical Biology, Cambridge University Press, 1988.

[93] C. van Vreeswijk and H. Sompolinsky, Chaos in neuronal networks with balanced excitatory and inhibitory activity, Science, 274, 1724-1726, 1996.

[94] C. van Vreeswijk and H. Sompolinsky, Chaotic balanced state in a model of cortical circuits, Neural Comput., 15, 1321-1371, 1998.

[95] T. Vogels and L. Abbott, Signal propagation and logic gating in networks of integrate-and-fire neurons., J. Neurosci, 25, 10786-95, 2005.

[96] M. Volgushev, I. Kudryashov, M. Chistiakova, M. Mukovski, J. Niesmann and U.T. Eysel, Probability of transmitter release at neocortical synapses at different temperatures, J. Neurophysiol., 92, 212-220, 2004.

[97] E.W. Weisstein, Dawson's integral. From MathWorld-a Wolfram web resource, http://mathworld.wolfram.com/DawsonsIntegral.html.

[98] W. Wilbur and J. Rinzel, A theoretical basis for large coefficient of variation and bimodality in neuronal interspike interval distributions, J. Theor. Biol, 105, 345-368, 1983.

[99] W. Yin Jin, J. Xue Xu, Y. Wu, L. Hong and Y. Bing Wei, Crisis of interspike intervals in Hodgkin-Huxley model, Chaos, Solitons \& Fractals, 27, 952-958, 2006.

[100] D. Zhou and D. Cai, A note on the absence of chaos from IEGF models with infinitely fast conductance fast conductance, unpublished notes.

[101] D. Zhou, A.V. Rangan, Y. Sun and D. Cai, Network-induced chaos in integrate-and-fire neuronal ensembles, submitted to Phys. Rev. E, 2008. 